Tohoku Math. J.

68 (2016), 161-197

\title{
LARGE DEVIATION PRINCIPLES FOR GENERALIZED FEYNMAN-KAC FUNCTIONALS AND ITS APPLICATIONS
}

\author{
DaEhong Kim, Kazuhiro Kuwae And Yoshihiro TAWARA
}

(Received August 4, 2014, revised September 19, 2014)

\begin{abstract}
Large deviation principles of occupation distribution for generalized Feynman-Kac functionals are presented in the framework of symmetric Markov processes having doubly Feller or strong Feller property. As a consequence, we obtain the $L^{p}$-independence of spectral radius of our generalized Feynman-Kac functionals. We also prove Fukushima's decomposition in the strict sense for functions locally in the domain of Dirichlet form having energy measure of Dynkin class without assuming no inside killing.
\end{abstract}

1. Introduction and statement of results. This paper is a continuation of the previous work [12] by authors including the first and second named present authors. The subject of [12] is the $L^{p}$-independence of the spectral bound of Feynman-Kac semigroup by continuous additive functionals in the framework of symmetric doubly Feller or strong Feller processes. In this paper, we focus on the Donsker-Varadhan type large deviation theory for generalized Feynman-Kac semigroups including non-local part in the framework of symmetric doubly Feller or strong Feller processes. As a consequence, we can and do deduce $L^{p}$-independence of the spectral bounds of our generalized Feynman-Kac semigroups.

The Donsker-Varadhan type large deviation principle of the occupation distribution for Markov processes has been developed by several authors. First, Fukushima-Takeda [17] gave a lower estimate in the Donsker-Varadhan type large deviation principle of the occupation distribution for Markov processes associated with irreducible Dirichlet forms admitting explosion or inside killing. Takeda [32] also gave an upper estimate in the Donsker-Varadhan type large deviation principle provided the underlying process is an irreducible Feller process satisfying the absolute continuity condition. In [33], Takeda extended the result in [32] to Feynman-Kac semigroups with continuous additive functionals of bounded variation. In Kim [19], this work was extended to non-local Feynman-Kac semigroups with discontinuous additive functionals in the extended Kato class. In [34] or [35], Takeda gave a sufficient

2010 Mathematics Subject Classification. Primary 31C25; Secondary 35B50, 60J45, 35J, 53C, 58.

Key words and phrases. Large deivation principle, Feynman-Kac semigroup, symmetric Markov processes, Dirichlet forms, occupation distribution, spectral bound, additive functional, continuous additive functional of zero energy, Kato class, local Kato class, extended Kato class, Feller property, strong Feller property, doubly Feller property.

The first named author was partially supported by a Grant-in-Aid for Scientific Research (C) No. 23540147 from Japan Society for the Promotion of Science. The second named author was partially supported by a Grant-in-Aid for Scientific Research (B) No. 22340036 from Japan Society for the Promotion of Science. The third named author was partially supported by a Grant-in-Aid for Young Scientists (B) No. 24740093 from Japan Society for the Promotion of Science. 
condition for the full large deviation principle for the occupation distribution of Markov processes and the $L^{p}$-independence of the spectral radius for symmetric Markov processes under this condition. In Takeda [36], he established a necessary and sufficient condition for the $L^{p}$-independence of spectral bounds for Feynman-Kac semigroups with continuous additive functionals of bounded variation whose Revuz measures are in the Kato class with 0-order Green-tightness in the framework of symmetric conservative Markov processes. The method of [36] also depends on the Donsker-Varadhan large deviation theory and remains valid for non-local Feynman-Kac semigroups (see [39, 42, 43]). They assumed the transience of the underlying process to define 0 -order Green-tight measures of Kato class. Without using the Feller property of the underlying process, Takeda [37] gave sufficient conditions for the $L^{p}$ independence of the spectral radius for Feynman-Kac semigroups with positive continuous additive functionals whose Revuz measure is in the Kato class with Green-tightness if the symmetrizing measure is positive-order Green-tight. Moreover, Takeda proved that the $L^{p}$ independence of the spectral radius is equivalent to the non-positivity of the $L^{2}$-spectral radius in the framework of one-dimensional diffusion processes on the interval having natural boundary (Theorem 5.1 in [37]).

For Feynman-Kac semigroups with continuous additive functionals of zero energy, Takeda and Zhang [41] obtained its asymptotic behavior based on the Donsker-Varadhan large deviation theory for $d$-dimensional Brownian motion. Zhang [45] obtained the asymptotic behavior of Feynman-Kac semigroups with continuous additive functionals of zero energy for symmetric Lévy processes under some conditions. In [12], the authors extended these results and also obtained the $L^{p}$-independence of the spectral radius of the Feynman-Kac semigroup for symmetric doubly Feller or strong Feller processes.

Very recently, Chen $[3,4]$ gives a general result on the $L^{p}$-independence of the spectral radius of the non-local Feynman-Kac semigroup involving continuous additive functionals of zero energy without using the large deviation theory. It is remarkable that in $[3,4]$ the doubly Feller nor strong Feller property of the underlying process are not imposed.

In this paper, we expose the large deviation principle for generalized Feynman-Kac functionals in the framework of doubly Feller or strong Feller symmetric Markov processes, which is extended to the all results in $[12,33,34,35,37,38,39,42,43]$. In particular, in the framework of strong Feller process, Theorem 1.1 with Remark 4.1(2) extends [37, 43], [38, Theorem 1.2], more precisely, the conclusions of Theorems 1.1 and 1.2(1) hold for our FeynmanKac functionals of additive functionals having Kato class conditions without Green-tightness. Our conditions on the measures related to our generalized Feynman-Kac functional are very mild for deducing the large deviation principle in terms of the given topology over the underlying space, however, it is somewhat restrictive for the $L^{p}$-independence of the spectral radius of it as indicated in [3, 4].

Now we state our framework and results. Let $E$ be a locally compact separable metric space and $m$ a positive Radon measure on $E$ with full topological support. Let $\mathbf{X}=$ $\left(\Omega, \mathcal{F}_{\infty}, \mathcal{F}_{t}, X_{t}, \zeta, \mathbf{P}_{x}, x \in E\right)$ be an $m$-symmetric Hunt process on $E$ and $(\mathcal{E}, \mathcal{F})$ the associated symmetric Dirichlet form on $L^{2}(E ; m)$. Let $\partial$ be a point added to $E$ so that $E_{\partial}:=E \cup\{\partial\}$ 
is the one-point compactification of $E$. The point $\partial$ also serves as the cemetery point for $\mathbf{X}$. For notational convenience, denote by $\mathbf{1}_{E}$ the constant function 1 on $E$, which vanishes at $\partial$, and by $\mathbf{1}_{E_{\partial}}$ the constant function 1 defined on $E_{\partial}$. Throughout this paper, we assume that $(\mathcal{E}, \mathcal{F})$ is irreducible, that is, any $\left(T_{t}\right)_{t>0}$-invariant set $B$ satisfies $m(B)=0$ or $m\left(B^{c}\right)=0$. Here $\left(T_{t}\right)_{t>0}$ is the strongly continuous semigroup on $L^{2}(E ; m)$ associated with $(\mathcal{E}, \mathcal{F})$. The transition kernel of $\mathbf{X}$ is denoted by $P_{t}(x, \mathrm{~d} y), t>0$. The correspondence between $\mathbf{X}$ and $(\mathcal{E}, \mathcal{F})$ is given by

$$
T_{t} f(x)=\mathbf{E}_{x}\left[f\left(X_{t}\right)\right]:=\int_{E} f(y) P_{t}(x, \mathrm{~d} y) \quad m \text {-a.e. } x \in E
$$

for each $t>0$ and any Borel function $f \in L^{2}(E ; m)$. The process $\mathbf{X}$ is said to have the Feller property if $P_{t}\left(C_{\infty}(E)\right) \subset C_{\infty}(E)$ for every $t>0$ and $\lim _{t \rightarrow 0}\left\|P_{t} f-f\right\|_{\infty}=0$ for every $f \in C_{\infty}(E)$. Here $C_{\infty}(E)$ is the space of continuous functions on $E$ that vanish at infinity and $\|f\|_{\infty}:=\sup _{x \in E}|f(x)|$. Under the Feller property of $\mathbf{X}$, for any compact subset $K$ of $E, \lim _{x \rightarrow \partial} \mathbf{E}_{x}\left[e^{-\alpha \sigma_{K}}\right]=0$ for each $\alpha>0$ and $\lim _{x \rightarrow \partial} \mathbf{P}_{x}\left(\sigma_{K} \leq t\right)=0$ for each $t>0$ (see Proposition 3.1 in [1], [42]). The space of bounded continuous functions on $E$ will be denoted as $C_{b}(E)$. The process $\mathbf{X}$ is said to have the strong Feller property if $P_{t}\left(\mathcal{B}_{b}(E)\right) \subset C_{b}(E)$ for every $t>0$. We say that $\mathbf{X}$ (or its transition semigroup) has the doubly Feller property if it has both the Feller and strong Feller properties. Throughout this paper, we assume that $\mathbf{X}$ has doubly Feller property. The Feller property of $\mathbf{X}$ yields the regularity of the Dirichlet form $(\mathcal{E}, \mathcal{F})$ on $L^{2}(E ; m)$. Since $\mathbf{X}$ has strong Feller property, $E$ is connected under the irreducibility of $(\mathcal{E}, \mathcal{F})$ and the transition kernel $\left(P_{t}\right)_{t>0}$ of $\mathbf{X}$ satisfies the absolute continuity condition with respect to $m$ ((AC) for short in this paper), i.e., $P_{t}(x, N)=0$ if $m(N)=0$ for each $N \in \mathcal{B}(E), x \in E$ and $t>0$.

For $\alpha>0$, there exists an $\alpha$-order resolvent kernel $R_{\alpha}(x, y)$ which is defined for all $x, y \in E$ (see Lemma 4.2.4 in [16]). Since $\alpha \mapsto R_{\alpha}(x, y)$ is decreasing for each $x, y \in E$, we can define the 0 -order resolvent kernel $R(x, y):=R_{0}(x, y):=\lim _{\alpha \rightarrow 0} R_{\alpha}(x, y)$. For a non-negative Borel measure $v$, we write $R_{\alpha} v(x):=\int_{E} R_{\alpha}(x, y) v(\mathrm{~d} y)$ and $R v(x):=R_{0} v(x)$. Note that $R_{\alpha} f(x)=R_{\alpha}(f m)(x)$ for any $f \in \mathcal{B}_{+}(E)$ or $f \in \mathcal{B}_{b}(E)$. A non-negative Borel measure $v$ is said to be of Dynkin class (resp. Kato class) if $\sup _{x \in E} R_{\alpha} v(x)<\infty$ for some $\alpha>0$ (resp. $\lim _{\alpha \rightarrow \infty} \sup _{x \in E} R_{\alpha} v(x)=0$ ), and $v$ is in the local Kato class if $\mathbf{1}_{K} v$ is in the Kato class for every compact set $K \subset E$. A non-negative Borel measure $v$ is said to be of extended Kato class if $\lim _{\alpha \rightarrow \infty} \sup _{x \in E} R_{\alpha} v(x)<1$. Denote by $S_{D}^{0}(\mathbf{X})\left(\operatorname{resp} . S_{K}^{0}(\mathbf{X})\right)$ the family of measures of Dynkin class (resp. Kato class) and by $S_{E K}^{0}(\mathbf{X})\left(\operatorname{resp} S_{L K}^{0}(\mathbf{X})\right)$ the family of measures of extended Kato class (resp. local Kato class). Clearly, $S_{K}^{0}(\mathbf{X}) \subset$ $S_{E K}^{0}(\mathbf{X}) \subset S_{D}^{0}(\mathbf{X})$ and $S_{K}^{0}(\mathbf{X}) \subset S_{L K}^{0}(\mathbf{X})$.

Denote by $S(\mathbf{X})$ (resp. $S_{1}(\mathbf{X}), S_{0}(\mathbf{X}), S_{00}(\mathbf{X})$ ) the family of smooth measures (resp. the family of smooth measures in the strict sense, the family of measures of finite energy integrals, the family of measures of finite energy integrals with bounded potentials) with respect to $\mathbf{X}$ (see pp. 83, pp. 79, (2.2.10) and pp. 238 in [16]). Note that any Radon measure of Dynkin class (hence any Radon measure of local/extended Kato class) always belongs to $S_{1}(\mathbf{X})$ in 
view of Proposition 3.1 in [22]. (Though the framework of [22] requires the existence of heat kernel, the proof of Proposition 3.1 in [22] still works in the present context.) We set $S_{D}^{1}(\mathbf{X}):=S_{D}^{0}(\mathbf{X}) \cap S_{1}(\mathbf{X}), S_{K}^{1}(\mathbf{X}):=S_{K}^{0}(\mathbf{X}) \cap S_{1}(\mathbf{X}), S_{E K}^{1}(\mathbf{X}):=S_{E K}^{0}(\mathbf{X}) \cap S_{1}(\mathbf{X})$ and $S_{L K}^{1}(\mathbf{X}):=S_{L K}^{0}(\mathbf{X}) \cap S_{1}(\mathbf{X})$. Conversely, any element $v \in S_{D}^{0}(\mathbf{X}) \cap S(\mathbf{X})$ is always a Radon measure by the regularity of the Dirichlet form $(\mathcal{E}, \mathcal{F})$ with the Stollmann-Voigt inequality (see [31])

$$
\int_{E} f^{2} \mathrm{~d} v \leq\left\|R_{\alpha} v\right\|_{\infty} \mathcal{E}_{\alpha}(f, f), \quad f \in \mathcal{F} .
$$

For each $\alpha \geq 0$, a positive measure $v \in S_{K}^{0}(\mathbf{X})$ is said to be $\alpha$-order Green-tight if and only if for any $\varepsilon>0$ there exists a compact subset $K$ of $E$ such that

$$
\sup _{x \in E} R_{\alpha}\left(\mathbf{1}_{K^{c}} v\right)(x)=\sup _{x \in E} \int_{K^{c}} R_{\alpha}(x, y) v(\mathrm{~d} y)<\varepsilon .
$$

By definition, for positive $\alpha, \alpha$-order Green-tightness of the measure $v \in S_{K}^{0}(\mathbf{X})$ is independent of the choice of $\alpha>0$ in view of the resolvent equation (4.2.12) in [16]. The measure of 0 -order Green-tightness is suitable to treat the transient case. In this case the 0-order Green-tightness always implies the $\alpha$-order Green-tightness for $\alpha>0$. Denote by $S_{K_{\infty}^{+}}^{0}(\mathbf{X})$ (resp. $S_{K_{\infty}}^{0}(\mathbf{X})$ ) the family of positive-order (resp. 0-order) Green-tight measures in the Kato class and set $S_{K_{\infty}^{+}}^{1}(\mathbf{X}):=S_{K_{\infty}^{+}}^{0}(\mathbf{X}) \cap S_{1}(\mathbf{X})\left(\operatorname{resp} . S_{K_{\infty}}^{1}(\mathbf{X}):=S_{K_{\infty}}^{0}(\mathbf{X}) \cap S_{1}(\mathbf{X})\right)$.

Let $\left(\mathcal{E}, \mathcal{F}_{e}\right)$ be the extended Dirichlet space of $(\mathcal{E}, \mathcal{F})$ (see [16] for the definition). Each element $f \in \mathcal{F}_{e}$ admits $\mathcal{E}$-quasi continuous $m$-version $\tilde{f}$ (see [16]). Throughout this paper, we always take $\mathcal{E}$-quasi continuous $m$-versions of elements of $\mathcal{F}_{e}$, that is, we omit tilde from $\tilde{f}$ for $f \in \mathcal{F}_{e}$.

Since $\mathbf{X}$ is a Hunt process, its Lévy system $\left(N(x, \mathrm{~d} y), H_{t}\right)$ exists and is defined under $\mathbf{P}_{x}$ for every $x \in E$, where $N(x, \mathrm{~d} y)$ is a kernel on $\left(E_{\partial}, \mathcal{B}\left(E_{\partial}\right)\right)$ and $H_{t}$ is a PCAF with bounded 1-potential such that for any nonnegative Borel function $\phi$ on $E_{\partial} \times E_{\partial}$ vanishing on the diagonal and any $x \in E_{\partial}$,

$$
\mathbf{E}_{x}\left[\sum_{s \leq t} \phi\left(X_{s-}, X_{s}\right)\right]=\mathbf{E}_{x}\left[\int_{0}^{t} \int_{E_{\partial}} \phi\left(X_{s}, y\right) N\left(X_{s}, \mathrm{~d} y\right) \mathrm{d} H_{s}\right] .
$$

To simplify notation, we will write

$$
N \phi(x):=\int_{E_{\partial}} \phi(x, y) N(x, \mathrm{~d} y) .
$$

Let $\mu_{H}$ be the Revuz measure of the PCAF $H$. Then the jumping measure $J$ and the killing measure $\kappa$ of $\mathbf{X}$ are given by

$$
J(\mathrm{~d} x \mathrm{~d} y)=\frac{1}{2} N(x, \mathrm{~d} y) \mu_{H}(\mathrm{~d} x), \quad \text { and } \quad \kappa(\mathrm{d} x)=N(x,\{\partial\}) \mu_{H}(\mathrm{~d} x) .
$$

These measures feature in the Beurling-Deny decomposition of $\mathcal{E}$ : for $f, g \in \mathcal{F}_{e}$,

$$
\mathcal{E}(f, g)=\mathcal{E}^{c}(f, g)+\int_{E \times E}(f(x)-f(y))(g(x)-g(y)) J(\mathrm{~d} x \mathrm{~d} y)+\int_{E} f(x) g(x) \kappa(\mathrm{d} x),
$$


where $\mathcal{E}^{c}$ is the strongly local part of $\mathcal{E}$.

Now we consider a bounded symmetric function $F$ on $E \times E_{\partial}$ which is extended to a function $F$ defined on $E_{\partial} \times E_{\partial}$ vanishing on the diagonal set of $E_{\partial} \times E_{\partial}$ (actually there is no need to define the value $F(\partial, y)$ for $y \in E)$. We say that $F$ is in the class $J_{1}(\mathbf{X})$ if the measure $N(|F|) \mu_{H}$ belongs to the class $S_{1}(\mathbf{X})$. It is said that $F$ is to be in the class $J_{D}^{1}(\mathbf{X})$ (resp. $J_{K}^{1}(\mathbf{X}), J_{L K}^{1}(\mathbf{X})$ and $\left.J_{E K}^{1}(\mathbf{X})\right)$ if the measure $N(|F|) \mu_{H}$ belongs to the class $S_{D}^{1}(\mathbf{X})$ (resp. $S_{K}^{1}(\mathbf{X}), S_{L K}^{1}(\mathbf{X})$ and $S_{E K}^{1}(\mathbf{X})$ ). For $\alpha \geq 0, F \in J_{K}^{1}(\mathbf{X})$ is said to be a function of $\alpha$-order Green-tightness if the measure $N(|F|) \mu_{H}$ belongs to the class $S_{K_{\infty}^{+}}^{1}(\mathbf{X})$. Denote by $J_{K_{\infty}^{+}}^{1}(\mathbf{X})$ (resp. $J_{K_{\infty}}^{1}(\mathbf{X})$ ) the family of functions of positive-order (resp. 0-order) Green-tightness.

For $F \in J_{1}(\mathbf{X})$, the following $A^{F}$ can be defined as an AF in the strict sense:

$$
A_{t}^{F}=\sum_{0<s \leq t} F\left(X_{s-}, X_{s}\right) .
$$

Note that $A_{t}^{F}=\sum_{0<s \leq t} \mathbf{1}_{\{s<\zeta\}} F\left(X_{s-}, X_{s}\right)$ provided $F(x, \partial)=0$ for $x \in E_{\partial}$. Hereafter, we take non-negative bounded functions $F_{1}, F_{2} \in J_{1}(\mathbf{X})$ on $E_{\partial} \times E_{\partial}$ vanishing on diagonal satisfying $\left\|F_{2}\right\|_{\infty}<1$ and $F_{1}(x, \partial)=F_{2}(x, \partial)=0, x \in E_{\partial}$, and set $F:=F_{1}-F_{2}$. Note that $F_{1} \geq F_{+}:=\max \{F, 0\}$ and $F_{2} \geq F_{-}:=\max \{-F, 0\}$ on $E_{\partial} \times E_{\partial}$.

A function $f$ on $E$ is said to be locally in $\mathcal{F}$ in the broad sense if there exists a nest $\left\{G_{n}\right\}$ of finely open (nearly) Borel sets and a sequence $\left\{f_{n}\right\}$ of elements in $\mathcal{F}$ such that $f=f_{n}$ $m$-a.e. on $G_{n}$. Let $\dot{\mathcal{F}}_{\text {loc }}$ be the family of functions on $E$ locally in $\mathcal{F}$ in the broad sense. It is known that any $f \in \dot{\mathcal{F}}_{\text {loc }}$ admits an $\mathcal{E}$-quasi continuous $m$-version. We introduce a subclass $\dot{\mathcal{F}}_{\text {loc }}^{\dagger}$ of $\dot{\mathcal{F}}_{\text {loc }}$ as follows:

$$
\dot{\mathcal{F}}_{\text {loc }}^{\dagger}:=\left\{f \in \dot{\mathcal{F}}_{\text {loc }} \mid N\left(\mathbf{1}_{E \times E}(f(\cdot)-f)^{2}\right) \mu_{H} \in S(\mathbf{X})\right\} .
$$

Clearly, $\dot{\mathcal{F}}_{\text {loc }}^{\dagger}$ is a linear subspaces of $\dot{\mathcal{F}}_{\text {loc }}$, and $\mathbf{1}_{E_{\partial}}, \mathbf{1}_{E} \in \dot{\mathcal{F}}_{\text {loc }}^{\dagger}$. By Remark 3.9 of [6] and $\kappa \in S$, we see $\mathcal{F}_{e} \cup\left(\dot{\mathcal{F}}_{\text {loc }}\right)_{b} \subset \dot{\mathcal{F}}_{\text {loc }}^{\dagger}$. For $f, g \in \dot{\mathcal{F}}_{\text {loc }}^{\dagger}$, we see $f g \in \dot{\mathcal{F}}_{\text {loc }}^{\dagger}$ provided $f$ or $g$ is bounded. Moreover, we define

$$
\mathcal{F}_{\text {loc }}^{\dagger}:=\left\{f \in \mathcal{F}_{\text {loc }} \mid \text { for any compact set } K, \int_{K \times E}(f(y)-f(x))^{2} J(\mathrm{~d} x \mathrm{~d} y)<\infty\right\} .
$$

Here $\mathcal{F}_{\text {loc }}$ is the space of functions locally in $\mathcal{F}$ in the ordinary sense (see [16]). Clearly, $\mathcal{F}_{\text {loc }}^{\dagger} \subset \dot{\mathcal{F}}_{\text {loc }}^{\dagger}$. For $f \in \mathcal{F}_{\text {loc }}, f \in \mathcal{F}_{\text {loc }}^{\dagger}$ if and only if that for any compact set $K$ with its relatively compact open neighborhood $G$

$$
\int_{K \times G^{c}}(f(y)-f(x))^{2} J(\mathrm{~d} x \mathrm{~d} y)<\infty .
$$

We see $\mathcal{F} \cup\left(\mathcal{F}_{\text {loc }}\right)_{b} \subset \mathcal{F}_{\text {loc }}^{\dagger}$, because of $J\left(K \times G^{c}\right)<\infty$, where $K$ and $G$ are noted as above.

REMARK 1.1. In [25], we introduce the following classes

$\dot{\mathcal{F}}_{\text {loc }}^{\ddagger}:=\left\{f \in \dot{\mathcal{F}}_{\text {loc }}^{\dagger} \mid f(\partial) \in \mathbb{R}\right.$ and $\left.(f(\cdot)-f(\partial))^{2} \kappa \in S(\mathbf{X})\right\}$,

$\mathcal{F}_{\text {loc }}^{\ddagger}:=\left\{f \in \mathcal{F}_{\text {loc }}^{\dagger} \mid f(\partial) \in \mathbb{R}\right.$ and for any compact set $\left.K, \int_{K}(f(x)-f(\partial))^{2} \kappa(\mathrm{d} x)<\infty\right\}$. 
These classes are unnecessary. Indeed, we can easily see that any $f \in \dot{\mathcal{F}}_{\text {loc }}^{\dagger}$ (resp. $f \in \mathcal{F}_{\text {loc }}^{\dagger}$ ) with $f(\partial) \in \mathbb{R}$ satisfies $(f(\cdot)-f(\partial))^{2} \kappa \in S(\mathbf{X})$ (resp. $\int_{K}(f(x)-f(\partial))^{2} \kappa(\mathrm{d} x)<\infty$ for any compact set $K$ ).

Hereafter, we fix $u \in \dot{\mathcal{F}}_{\text {loc }} \cap C\left(E_{\partial}\right)$ with $\mu_{\langle u\rangle} \in S_{K}^{1}(\mathbf{X})$. In Theorem 6.2(2) below, we prove that the additive functional $u\left(X_{t}\right)-u\left(X_{0}\right)$ admits the following strict decomposition:

$$
u\left(X_{t}\right)-u\left(X_{0}\right)=M_{t}^{u}+N_{t}^{u} \quad t \in\left[0,+\infty\left[\quad \mathbf{P}_{x} \text {-a.s. for all } x \in E,\right.\right.
$$

where $M^{u}$ is a square integrable martingale additive functional in the strict sense, and $N^{u}$ is a continuous additive functional in the strict sense. $M^{u}$ can be decomposed as

$$
M_{t}^{u}=M_{t}^{u, c}+M_{t}^{u, j}+M_{t}^{u, \kappa},
$$

where $M_{t}^{u, j}, M_{t}^{u, \kappa}$ and $M_{t}^{u, c}$ are the jumping, killing and continuous part of $M^{u}$ respectively. Those are defined $\mathbf{P}_{x}$-a.s. for all $x \in E$ by Theorem 6.2(2). The strict decompositions (1.1) and (1.2) are necessary to deduce the large deviation principle for our Feynman-Kac semigroup defined later.

Let $\mu_{\langle u\rangle}, \mu_{\langle u\rangle}^{c}, \mu_{\langle u\rangle}^{j}$ and $\mu_{\langle u\rangle}^{\kappa}$ be the smooth Revuz measures in the strict sense associated with the quadratic variational processes (or the sharp bracket PCAFs in the strict sense) $\left\langle M^{u}\right\rangle$, $\left\langle M^{u, c}\right\rangle,\left\langle M^{u, j}\right\rangle$ and $\left\langle M^{u, \kappa}\right\rangle$ respectively. Then

$$
\mu_{\langle u\rangle}(\mathrm{d} x)=\mu_{\langle u\rangle}^{c}(\mathrm{~d} x)+\mu_{\langle u\rangle}^{j}(\mathrm{~d} x)+\mu_{\langle u\rangle}^{\kappa}(\mathrm{d} x) .
$$

Note that $\mathcal{E}(u, u)=\frac{1}{2} v_{\langle u\rangle}(E)$ with $v_{\langle u\rangle}:=\mu_{\langle u\rangle}^{c}+\mu_{\langle u\rangle}^{j}+2 \mu_{\langle u\rangle}^{\kappa}$ provided $u \in \mathcal{F}_{e}$.

Hereafter we use the convention that $\mu=\mu_{1}-\mu_{2} \in S_{*}^{1}(\mathbf{X})-S_{* *}^{1}(\mathbf{X})$ means $\mu_{1} \in S_{*}^{1}(\mathbf{X})$ and $\mu_{2} \in S_{* *}^{1}(\mathbf{X})$ for subclasses $S_{*}^{1}(\mathbf{X})$ and $S_{* *}^{1}(\mathbf{X})$ of $S_{1}(\mathbf{X})$, where $\mu=\mu_{1}-\mu_{2}$ is the Hahn-Jordan decomposition of the signed smooth measure $\mu$ in the strict sense. Similarly $F=F_{1}-F_{2} \in J_{*}^{1}(\mathbf{X})-J_{* *}^{1}(\mathbf{X})$ means $F_{1} \in J_{*}^{1}(\mathbf{X})$ and $F_{2} \in J_{* *}^{1}(\mathbf{X})$ for subclasses $J_{*}^{1}(\mathbf{X})$ and $J_{* *}^{1}(\mathbf{X})$ of $J_{1}(\mathbf{X})$.

We always take $\mu=\mu_{1}-\mu_{2}$ and $F=F_{1}-F_{2}$ with $\mu_{1}+N\left(F_{1}\right) \mu_{H} \in S_{L K}^{1}(\mathbf{X}) \cap S_{E K}^{1}(\mathbf{X})$, $\mu_{2}+N\left(F_{2}\right) \mu_{H} \in S_{L K}^{1}(\mathbf{X})$.

We now fix an AF $A:=N^{u}+A^{\mu, F}$ with $A^{\mu, F}:=A^{\mu}+A^{F}$. Here $A_{t}^{\mu}:=A_{t}^{\mu_{1}}-A_{t}^{\mu_{2}}$, and $A_{t}^{\mu_{1}}$ (resp. $A_{t}^{\mu_{2}}$ ) is the PCAF in the strict sense associated with $\mu_{1}$ (resp. $\mu_{2}$ ) as its Revuz measure.

Now we consider the following multiplicative functional:

$$
e_{A}(t):=\exp \left(N_{t}^{u}\right) \operatorname{Exp}\left(A^{\mu, F}\right)_{t}, \quad t \geq 0 .
$$

Here $\operatorname{Exp}\left(A^{\mu, F}\right)_{t}:=e^{A_{t}^{\mu}} \Pi_{s \leq t}\left(1+F\left(X_{s-}, X_{S}\right)\right)$ is the Stieltjes exponential of $A_{t}^{\mu, F}$. Define the associated Feynman-Kac semigroup by

$$
Q_{t} f(x):=\mathbf{E}_{x}\left[e_{A}(t) f\left(X_{t}\right)\right] \quad \text { for } x \in E, f \in \mathcal{B}_{+}(E) .
$$

The probabilistic resolvent $\left(S_{\alpha}\right)_{\alpha>0}$ associated with $\left(Q_{t}\right)_{t>0}$ is defined by

$$
S_{\alpha} f(x):=\int_{0}^{\infty} e^{-\alpha t} Q_{t} f(x) \mathrm{d} t \quad x \in E
$$


for $f \in \mathcal{B}_{+}(E)$.

Define a transition probability $P_{t}^{\partial}(x, \mathrm{~d} y)$ on $E_{\partial}$; for $B \in \mathcal{B}\left(E_{\partial}\right)$,

$$
P_{t}^{\partial}(x, B)= \begin{cases}P_{t}(x, B \backslash\{\partial\}) & x \in E, \\ \delta_{\partial}(B) & x=\partial .\end{cases}
$$

Denote by $\mathbf{X}_{\partial}=\left(X_{t}, \mathbf{P}_{x}^{\partial}, x \in E_{\partial}\right)$ the Markov process with transition probability $P_{t}^{\partial}(x, \mathrm{~d} y)$. $\mathbf{X}_{\partial}$ is an extension of $\mathbf{X}$ with $\partial$ as a cemetery point. We also use that for $f \in \mathcal{B}_{+}\left(E_{\partial}\right)$ and $x \in E_{\partial}$,

$$
Q_{t}^{\partial} f(x):=\mathbf{E}_{x}^{\partial}\left[e_{A}(t) f\left(X_{t}\right)\right] \quad \text { and } \quad S_{\alpha}^{\partial} f(x):=\int_{0}^{\infty} e^{-\alpha t} Q_{t}^{\partial} f(x) \mathrm{d} t .
$$

Any function $f$ on $E$ can be regarded as a function $f$ on $E_{\partial}$ with $f(\partial)=0$ unless we mention the value at infinity. In this case $Q_{t} f(x)=Q_{t}^{\partial} f(x)$ for $x \in E$ and $f \in \mathcal{B}(E)$. In particular, $Q_{t}^{\partial} \mathbf{1}_{E_{\partial}}(x)=\mathbf{E}_{x}\left[e_{A}(t)\right]$ and $Q_{t}^{\partial} \mathbf{1}_{E}(x)=Q_{t} \mathbf{1}_{E}(x)=\mathbf{E}_{x}\left[e_{A}(t): t<\zeta\right], x \in E$.

Let $\mathcal{C}$ be a core of $(\mathcal{E}, \mathcal{F})$ on $L^{2}(E ; m)$. Under the above conditions for $\mu_{\langle u\rangle}, \mu$ and $F$, we introduce the following quadratic form $(\mathcal{Q}, \mathcal{C})$ on $L^{2}(E ; m)$ : for $f, g \in \mathcal{C}$

$$
\mathcal{Q}(f, g):=\mathcal{E}(f, g)+\mathcal{E}(u, f g)-\mathcal{H}(f, g),
$$

where

$$
\begin{aligned}
\mathcal{E}(u, f g) & :=\frac{1}{2} \mu_{\langle u, f g\rangle}^{c}(E)+\int_{E \times E}(u(x)-u(y))((f g)(x)-(f g)(y)) J(\mathrm{~d} x \mathrm{~d} y)+\int_{E} u f g \mathrm{~d} \kappa, \\
\mathcal{H}(f, g) & :=\int_{E} f(x) g(x) \mu(\mathrm{d} x)+\iint_{E \times E} f(x) g(y) F(x, y) N(x, \mathrm{~d} y) \mu_{H}(\mathrm{~d} x) .
\end{aligned}
$$

Under the condition $\mu_{\langle u\rangle} \in S_{K}^{1}(\mathbf{X})$ (resp. $\mu_{1}+N\left(F_{1}\right) \mu_{H} \in S_{L K}^{1}(\mathbf{X}) \cap S_{E K}^{1}(\mathbf{X}), \mu_{2}+$ $\left.N\left(F_{2}\right) \mu_{H} \in S_{L K}^{1}(\mathbf{X})\right)$, the quantity $\mathcal{E}(u, f g)$ (resp. $\left.\mathcal{H}(f, g)\right)$ is well-defined for $f, g \in \mathcal{C}$, consequently, the quadratic form $\mathcal{Q}$ is well defined and lower bounded on $\mathcal{C}$ and the probabilistic semigroup defined in (1.3) is regarded as a strongly continuous semigroup on $L^{2}(E ; m)$ associated with the closure $(\mathcal{Q}, D(\mathcal{Q}))$ of $(\mathcal{Q}, \mathcal{C})$ on $L^{2}(E ; m)$ (Corollaries 1.5, 1.8 and 1.9 in [7]). Note that $D(\mathcal{Q}) \subset \mathcal{F}$ always holds, because there exist $C>0$ and $\alpha_{0}>0$ such that $C^{-1} \mathcal{E}_{1}(f, f) \leq \mathcal{Q}_{\alpha_{0}}(f, f)$ for all $f \in D(\mathcal{Q})$. Moreover, if further $\mu_{2}+N\left(F_{2}\right) \mu_{H} \in$ $S_{L K}^{1}(\mathbf{X}) \cap S_{D}^{1}(\mathbf{X})$, then there exist $C>0$ and $\alpha_{0}>0$ such that

$$
C^{-1} \mathcal{E}_{1}(f, f) \leq \mathcal{Q}_{\alpha_{0}}(f, f) \leq C \mathcal{E}_{1}(f, f) \text { for all } f \in \mathcal{F} .
$$

In this case, we have $D(\mathcal{Q})=\mathcal{F}$.

We state some notations. Let $\mathcal{P}(E)$ denote the space of all Borel probability measures on $E$. Define a rate function $I_{\mathcal{Q}}(v)$ on $\mathcal{P}(E)$ by

$$
I_{\mathcal{Q}}(v):= \begin{cases}\mathcal{Q}(\phi, \phi) & \text { if } v \ll m \text { and } \phi:=\sqrt{\mathrm{d} v / \mathrm{d} m} \in \mathcal{D}(\mathcal{Q}) \\ +\infty & \text { otherwise }\end{cases}
$$


For $\omega \in \Omega$ with $t<\zeta(\omega)$, we define the normalized occupation time distribution $L_{t}(\omega) \in$ $\mathcal{P}(E)$ by

$$
L_{t}(\omega)(A):=\frac{1}{t} \int_{0}^{t} \mathbf{1}_{A}\left(X_{s}(\omega)\right) \mathrm{d} s \quad \text { for } A \in \mathcal{B}(E) .
$$

Our first result is the following:

THEOREM 1.1. Suppose $\mu_{\langle u\rangle} \in S_{K}^{1}(\mathbf{X}), \mu=\mu_{1}-\mu_{2}$ and $F=F_{1}-F_{2}$ with $\mu_{1}+$ $N\left(F_{1}\right) \mu_{H} \in S_{L K}^{1}(\mathbf{X}) \cap S_{E K}^{1}(\mathbf{X}), \mu_{2}+N\left(F_{2}\right) \mu_{H} \in S_{L K}^{1}(\mathbf{X})$.

(1) For any open set $G \subset \mathcal{P}(E)$ and $x \in E$,

$$
\varliminf_{t \rightarrow \infty} \frac{1}{t} \log \mathbf{E}_{x}\left[e_{A}(t): L_{t} \in G, t<\zeta\right] \geq-\inf _{\nu \in G} I_{\mathcal{Q}}(v) .
$$

(2) Assume $\mu_{2}+N\left(F_{2}\right) \mu_{H} \in S_{L K}^{1}(\mathbf{X}) \cap S_{D}^{1}(\mathbf{X})$. Then for any compact set $K \subset \mathcal{P}(E)$,

$$
\varlimsup_{t \rightarrow \infty} \frac{1}{t} \log \sup _{x \in E} \mathbf{E}_{x}\left[e_{A}(t): L_{t} \in K, t<\zeta\right] \leq-\inf _{v \in K} I_{\mathcal{Q}}(v) .
$$

(3) Assume further $m \in S_{K_{\infty}^{+}}^{1}(\mathbf{X})$ and $\mu_{2}+N\left(F_{2}\right) \mu_{H} \in S_{L K}^{1}(\mathbf{X}) \cap S_{D}^{1}(\mathbf{X})$. Then for any closed set $K \subset \mathcal{P}(E)$, we have (1.4). In particular,

$$
\begin{aligned}
& \lim _{t \rightarrow \infty} \frac{1}{t} \log \mathbf{E}_{x}\left[e_{A}(t): t<\zeta\right] \\
& \quad=\lim _{t \rightarrow \infty} \frac{1}{t} \log \sup _{x \in E} \mathbf{E}_{x}\left[e_{A}(t): t<\zeta\right]=-\inf _{v \in \mathcal{P}(E)} I_{\mathcal{Q}}(v) .
\end{aligned}
$$

Define the probability measure on $\mathcal{P}(E)$ by

$$
\mathbf{Q}_{x, t}(B):=\frac{\mathbf{E}_{x}\left[e_{A}(t): L_{t} \in B, t<\zeta\right]}{\mathbf{E}_{x}\left[e_{A}(t): t<\zeta\right]}, \quad B \in \mathcal{B}(\mathcal{P}(E)) .
$$

Define the function $J$ on $\mathcal{P}(E)$ by

$$
J(v):=I_{\mathcal{Q}}(v)-\lambda_{2}(u, \mu, F) .
$$

Here $\lambda_{2}(u, \mu, F):=\inf _{f \in \mathcal{F},\|f\|_{2}=1} \mathcal{Q}(f, f)$ is the bottom of the $L^{2}$-spectrum of our Feynman-Kac semigroup. We have the following:

Corollary 1.1. Suppose $\mu_{\langle u\rangle} \in S_{K}^{1}(\mathbf{X}), \mu=\mu_{1}-\mu_{2}$ and $F=F_{1}-F_{2}$ with $\mu_{1}+N\left(F_{1}\right) \mu_{H} \in S_{L K}^{1}(\mathbf{X}) \cap S_{E K}^{1}(\mathbf{X}), \mu_{2}+N\left(F_{2}\right) \mu_{H} \in S_{L K}^{1}(\mathbf{X}) \cap S_{D}^{1}(\mathbf{X})$. Assume $m \in$ $S_{K_{\infty}^{+}}^{1}(\mathbf{X})$.

(1) There exists a unique ground state, that is, there exists $u_{0} \in \mathcal{F}$ with $\left\|u_{0}\right\|_{2}=1$ such that $\mathcal{Q}\left(u_{0}, u_{0}\right)=\inf _{f \in \mathcal{F},\|f\|_{2}=1} \mathcal{Q}(f, f)$.

(2) $\left\{\mathbf{Q}_{x, t}\right\}_{t>0}$ obeys the large deviation principle with rate function $J$ :

(a) For any open set $G \subset \mathcal{P}(E)$

$$
\varliminf_{t \rightarrow \infty} \frac{1}{t} \log \mathbf{Q}_{x, t}(G) \geq-\inf _{v \in G} J(v) .
$$


(b) For any closed set $K \subset \mathcal{P}(E)$,

$$
\varlimsup_{t \rightarrow \infty} \frac{1}{t} \log \mathbf{Q}_{x, t}(K) \leq-\inf _{v \in K} J(v) .
$$

(3) The measure $\mathbf{Q}_{x, t}$ converges to $\delta_{u_{0}^{2} m}$ weakly.

Fix an open subset $G$ of $E$ and consider a part space $\left(\mathcal{E}_{G}, \mathcal{F}_{G}\right)$ defined by

$$
\left\{\begin{array}{l}
\mathcal{F}_{G}:=\{f \in \mathcal{F} \mid f=0 \text { q.e. on } E \backslash G\}, \\
\mathcal{E}_{G}(f, g):=\mathcal{E}(f, g) \text { for } f, g \in \mathcal{F}_{G} .
\end{array}\right.
$$

It is known that $\left(\mathcal{E}_{G}, \mathcal{F}_{G}\right)$ is a regular Dirichlet form on $L^{2}(G ; m)$. Under the doubly Feller property of $\mathbf{X}$, it is proved in [11, Corollary] that $R_{1}^{G} \mathbf{1}_{G} \in C_{\infty}(G)$ provided $G$ is a relatively compact open set which is regular in the sense that $\mathbf{P}_{x}\left(\tau_{G}=0\right)=1$ for all $x \in E \backslash G$. Here $R_{1}^{G}$ is the 1-order resolvent under the part process $\mathbf{X}_{G}$ and $\tau_{G}:=\inf \left\{t>0 \mid X_{t} \notin G\right\}$ is the first exit time from $G$. Consider a probabilistic semigroup $Q_{t}^{G} f(x):=\mathbf{E}_{x}\left[e_{A}(t) f\left(X_{t}\right): t<\tau_{G}\right]$ for $f \in \mathcal{B}_{b}(G)$. Note that $Q_{t}^{G}$ forms a strongly continuous semigroup on $L^{2}(G ; m)$ associated with a closed quadratic form $\left(\mathcal{Q}, \mathcal{F}_{G}\right)$ on $L^{2}(G ; m)$ provided $G$ is relatively compact.

Let us denote by $\left\|Q_{t}^{G}\right\|_{p, p}$ the operator norm of $Q_{t}^{G}$ from $L^{p}(G ; m)$ to $L^{p}(G ; m)$ and put

$$
\lambda_{p}(G):=\lambda_{p}(u, \mu, F)(G):=-\lim _{t \rightarrow \infty} \frac{1}{t} \log \left\|Q_{t}^{G}\right\|_{p, p}, \quad 1 \leq p \leq \infty
$$

and we omit ' $(G)$ ' from $\lambda_{p}(G)$ when $G=E$.

The following theorem is a direct consequence of Theorem 1.1.

THEOREM 1.2. Suppose $\mu_{\langle u\rangle} \in S_{K}^{1}(\mathbf{X}), \mu=\mu_{1}-\mu_{2}$ and $F=F_{1}-F_{2}$ with $\mu_{1}+$ $N\left(F_{1}\right) \mu_{H} \in S_{L K}^{1}(\mathbf{X}) \cap S_{E K}^{1}(\mathbf{X}), \mu_{2}+N\left(F_{2}\right) \mu_{H} \in S_{L K}^{1}(\mathbf{X}) \cap S_{D}^{1}(\mathbf{X})$.

(1) The spectral radius $\lambda_{p}(u, \mu, F)(1 \leq p \leq \infty)$ is independent of $p$ if $m \in S_{K_{\infty}^{+}}^{1}(\mathbf{X})$.

(2) The spectral radius $\lambda_{p}(u, \mu, F)(G)(1 \leq p \leq \infty)$ is independent of $p$ if $G$ is a regular open set satisfying $\lim _{G \ni x \rightarrow \partial} \mathbf{P}_{x}\left(\tau_{G}>0\right)=0$.

(3) Suppose the weaker conditions than the hypotheses of the present theorem that $\mu_{\langle u\rangle} \in$ $S_{L K}^{1}(\mathbf{X}), \mu=\mu_{1}-\mu_{2} \in S_{L K}^{1}(\mathbf{X})-S_{L K}^{1}(\mathbf{X})$ and $F=F_{1}-F_{2} \in J_{L K}^{1}(\mathbf{X})-J_{L K}^{1}(\mathbf{X})$.

Then the spectral radius $\lambda_{p}(u, \mu, F)(G)(1 \leq p \leq \infty)$ is independent of $p$ if $G$ is a relatively compact regular open set.

We further have the following:

THEOREM 1.3. Suppose $\mu_{\langle u\rangle} \in S_{K_{\infty}^{+}}^{1}(\mathbf{X}), \mu=\mu_{1}-\mu_{2} \in S_{K_{\infty}^{+}}^{1}(\mathbf{X})-S_{L K}^{1}(\mathbf{X}) \cap S_{D}^{1}(\mathbf{X})$ and $F=F_{1}-F_{2} \in J_{K_{\infty}^{+}}^{1}(\mathbf{X})-J_{L K}^{1}(\mathbf{X}) \cap J_{D}^{1}(\mathbf{X})$. Then the spectral radius $\lambda_{p}(u, \mu, F)(1 \leq$ $p \leq \infty)$ is independent of $p$ if $\lambda_{2}(u, \mu, F) \leq 0$. Moreover, suppose that $\mathbf{X}$ is conservative, $\mu_{2} \in S_{K_{\infty}^{+}}^{1}(\mathbf{X})$ and $F_{2} \in J_{K_{\infty}^{+}}^{1}(\mathbf{X})$. Then $\lambda_{2}(u, \mu, F)>0$ implies $\lambda_{\infty}(u, \mu, F)=0$.

COROLlary 1.2. Suppose $\mu_{\langle u\rangle} \in S_{K_{\infty}^{+}}^{1}(\mathbf{X}), \mu=\mu_{1}-\mu_{2}$ with $\mu_{1} \in S_{K_{\infty}^{+}}^{1}(\mathbf{X})$ and $\mu_{2}=0$, and assume $F=F_{1}-F_{2}$ with $F_{1} \in J_{K_{\infty}^{+}}^{1}(\mathbf{X})$ and $F_{2}=0$. Then $\lambda_{2}(0,0,0) \leq 0$ implies $\lambda_{2}(u, \mu, F) \leq 0$, in particular, $\lambda_{p}(u, \mu, F)(1 \leq p \leq \infty)$ is independent of $p$ if 
$\lambda_{2}(0,0,0) \leq 0$. Moreover, if $\mathbf{X}$ is transient, $\mu_{\langle u\rangle} \in S_{K_{\infty}^{+}}^{1}(\mathbf{X}), \mu=\mu_{1}-\mu_{2} \in S_{K_{\infty}^{+}}^{1}(\mathbf{X})-$ $S_{K_{\infty}}^{1}(\mathbf{X})$ and $F=F_{1}-F_{2} \in J_{K_{\infty}^{+}}^{1}(\mathbf{X})-J_{K_{\infty}}^{1}(\mathbf{X})$ then the same conclusion holds.

These results are extensions of $[12,34,35,36]$. Let us briefly state the constitution of this paper. In Section 3, we investigate the properties of our Feynman-Kac semigroup generated by continuous additive functionals whose Revuz measures are of (local and extended) Kato class and give more properties if they have the positive-order Green-tightness. In Section 4, we give the proofs of our main results. In Section 5, we give examples on birth and death processes (Example 5.1), on relativistic stable processes (Example 5.2). In Section 6, we restate Fukushima's decomposition in the strict sense up to infinity as an errata for the appendix in [12] and prove it without assuming no inside killing.

2. Properties on Green-tight PAF of Kato class. In this section, we use the terminologies additive functional ( $A F$ in short) in the strict sense and positive additive functional (PAF in short) in the strict sense, which are presented in [8]. A PAF $A$ in the strict sense is said to be of Kato class if $\sup _{x \in E} \mathbf{E}_{x}\left[\int_{0}^{\infty} e^{-\alpha t} \mathrm{~d} A_{t}\right] \rightarrow 0$ as $\alpha \rightarrow \infty$, equivalently $\sup _{x \in E} \mathbf{E}_{x}\left[A_{t}\right] \rightarrow 0$ as $t \rightarrow 0$. A PAF $A$ in the strict sense is said to be of Dynkin class if $\sup _{x \in E} \mathbf{E}_{x}\left[\int_{0}^{\infty} e^{-\alpha t} \mathrm{~d} A_{t}\right]<\infty$ for some/any $\alpha>0$, equivalently $\sup _{x \in E} \mathbf{E}_{x}\left[A_{t}\right]<\infty$ for some/any $t>0$. A PAF $A$ in the strict sense is said be of extended Kato class if $\lim _{t \rightarrow 0} \sup _{x \in E} \mathbf{E}_{x}\left[A_{t}\right]<1$. A PAF $A$ in the strict sense is said be of local Kato class if for any compact set $K,\left(\mathbf{1}_{K} * A\right)$, defined by $\left(\mathbf{1}_{K} * A\right)_{t}:=\int_{0}^{t} \mathbf{1}_{K}\left(X_{S}\right) \mathrm{d} A_{s}$, is of Kato class. A PAF $A$ in the strict sense is said be of positive order Green-tight Kato class if for some/any $\alpha>0$,

$$
\lim _{\substack{K \uparrow E \\ K: \text { compact }}} \sup _{x \in E} \mathbf{E}_{x}\left[\int_{0}^{\infty} e^{-\alpha t} \mathbf{1}_{K^{c}}\left(X_{t}\right) \mathrm{d} A_{t}\right]=0 .
$$

If $A$ is a PCAF in the strict sense, those above notions correspond the notions defined before with respect to its Revuz measure.

The following lemma can be similarly proved as for [12, Lemma 2.2]. So we omit its proof.

LEMMA 2.1. Let $A$ be an AF in the strict sense, which is a difference of PAFs in the strict sense. Let $A=A^{+}-A^{-}$be the Hahn-Jordan decomposition of $A$ and set $|A|:=$ $A^{+}+A^{-}$. Then $|A|$ is a PAF in the strict sense. For $p \in[1, \infty[$, we have

$$
\mathbf{E}_{x}\left[\left|A_{t}\right|^{p}\right] \leq C_{p}\left(\sup _{y \in E} \mathbf{E}_{y}\left[\left|A_{t}\right|\right]\right)^{[p]} \mathbf{E}_{x}\left[\left|A_{t}\right|\right]^{p-[p]} \leq C_{p}\left(\sup _{y \in E} \mathbf{E}_{y}\left[\left|A_{t}\right|\right]\right)^{p},
$$

where $[p]:=\sup \{x \in \mathbb{N} \mid x \leq p\}$ and $C_{p}:=p(p-1) \cdots(p-[p]+1)$. In particular, for PAFs $A$ and $B$ of Dynkin class, $x \mapsto \mathbf{E}_{x}\left[A_{t}^{p} B_{t}^{q}\right]$ is bounded Borel measurable for any $p, q \geq 0$.

Proposition 2.1. Let A, B be PAFs of Dynkin class and of local Kato class in the strict sense. Under the strong Feller property of $\mathbf{X}$, we have the following: 
(1) For each compact set $K, p \geq 1$ and $s \geq 0$,

$$
\lim _{t \rightarrow 0} \sup _{x \in K} \mathbf{E}_{x}\left[A_{t}^{p} \circ \theta_{s}\right]=0 .
$$

(2) Let $p, q \in \mathbb{N} \cup\{0\}$. Then $\mathbf{E} .\left[A_{t}^{p} B_{t}^{q}\right] \in C_{b}(E)$.

(3) Suppose that A belongs to the extended Kato class and B belongs to the Kato class. Then, for sufficiently small $t>0$, E. $\left[e^{A_{t}-B_{t}}\right] \in C_{b}(E)$.

Proof. (1): First we prove (2.1) for $s=0$. Let $\left\{G_{n}\right\}$ be an increasing sequence of relatively compact open sets with $K \subset G_{1}$. Owing to the strong Feller property of $\mathbf{X}$, we have $\sup _{x \in K} \mathbf{P}_{x}(\zeta \leq t) \rightarrow 0$ as $t \rightarrow 0$, and $\sup _{x \in K} \mathbf{P}_{x}\left(\tau_{G_{n}} \leq t<\zeta\right) \rightarrow 0$ as $n \rightarrow \infty$ for each $t>0$ (its proof can be seen after pp. 911 line -1 in [8]). Since $\left(\mathbf{1}_{G_{n}} * A\right)$ is of Kato class,

$$
\begin{aligned}
\lim _{t \rightarrow 0} \sup _{x \in K} \mathbf{E}_{x}\left[A_{t \wedge \tau_{G_{n}}}^{p}\right] & \leq \lim _{t \rightarrow 0} \sup _{x \in K} \mathbf{E}_{x}\left[\left(\int_{0}^{t} \mathbf{1}_{G_{n}}\left(X_{s}\right) \mathrm{d} A_{s}\right)^{p}\right] \\
& \leq C_{p} \lim _{t \rightarrow 0}\left(\sup _{x \in E} \mathbf{E}_{x}\left[\int_{0}^{t} \mathbf{1}_{G_{n}}\left(X_{s}\right) \mathrm{d} A_{s}\right]\right)^{p}=0 .
\end{aligned}
$$

Then we see

$$
\begin{aligned}
\lim _{t \rightarrow 0} \sup _{x \in K} \mathbf{E}_{x}\left[A_{t}^{p}\right] \leq & \lim _{t \rightarrow 0} \sup _{x \in K} \mathbf{E}_{x}\left[A_{t}^{p}: t \geq \zeta\right]+\lim _{t \rightarrow 0} \sup _{x \in K} \mathbf{E}_{x}\left[A_{t}^{p}-A_{t \wedge \tau_{G_{n}}}^{p}: t<\zeta\right] \\
\leq & \lim _{t \rightarrow 0} \sup _{x \in K} \mathbf{E}_{x}\left[A_{t}^{2 p}\right]^{1 / 2} \sup _{x \in K} \mathbf{P}_{x}(\zeta \leq t) \\
& +\lim _{t \rightarrow 0} \sup _{x \in K} \mathbf{E}_{x}\left[A_{t}^{p}-A_{t \wedge \tau_{G_{n}}}^{p}: \tau_{G_{n}} \leq t<\zeta\right] \\
\leq & \lim _{t \rightarrow 0} \sup _{x \in K} \mathbf{E}_{x}\left[A_{t}^{p}: \tau_{G_{n}} \leq t<\zeta\right] \\
= & \lim _{t \rightarrow 0} \sup _{x \in K} \mathbf{E}_{x}\left[A_{t}^{p}: \tau_{G_{n}} \leq t\right]-\lim _{t \rightarrow 0} \sup _{x \in K} \mathbf{E}_{x}\left[A_{t}^{p}: \zeta \leq t\right] \\
\leq & \sup _{x \in K} \mathbf{E}_{x}\left[A_{T}^{p}: \tau_{G_{n}} \leq T\right] \\
\leq & \sup _{x \in K} \mathbf{E}_{x}\left[A_{T}^{p}: \tau_{G_{n}} \leq T<\zeta\right]+\sup _{x \in K} \mathbf{E}_{x}\left[A_{T}^{p}: \zeta \leq T\right] \\
\leq & \sup _{x \in K} \mathbf{E}_{x}\left[A_{T}^{2 p}\right]^{1 / 2}\left(\sup _{x \in K} \mathbf{P}_{x}\left(\tau_{G_{n}} \leq T<\zeta\right)+\sup _{x \in K} \mathbf{P}_{x}(\zeta \leq T)\right) .
\end{aligned}
$$

Letting $n \rightarrow \infty$ and $T \rightarrow 0$, we have the assertion. Next we prove (2.1) for general $s>0$. Noting $A_{t} \circ \theta_{s}=0$ for $s \geq \zeta$, we have

$$
\begin{aligned}
& \lim _{t \rightarrow 0} \sup _{x \in K} \mathbf{E}_{x}\left[A_{t}^{p} \circ \theta_{s}\right]=\lim _{t \rightarrow 0} \sup _{x \in K} \mathbf{E}_{x}\left[A_{t}^{p} \circ \theta_{s}: s<\zeta\right] \\
& \quad \leq \lim _{t \rightarrow 0} \sup _{x \in K} \mathbf{E}_{x}\left[A_{t}^{p} \circ \theta_{s}: \tau_{G_{n}} \leq s<\zeta\right]+\lim _{t \rightarrow 0} \sup _{x \in K} \mathbf{E}_{x}\left[A_{t}^{p} \circ \theta_{s}: s<\tau_{G_{n}}\right] \\
& \quad \leq \lim _{t \rightarrow 0} \sup _{x \in K} \mathbf{E}_{x}\left[A_{t}^{2 p} \circ \theta_{s}\right]^{1 / 2} \sup _{x \in K} \mathbf{P}_{x}\left(\tau_{G_{n}} \leq s<\zeta\right)+\lim _{t \rightarrow 0} \sup _{x \in K} \mathbf{E}_{x}\left[\mathbf{E}_{X_{s}}\left[A_{t}^{p}\right]: s<\tau_{G_{n}}\right] \\
& \quad \leq \lim _{t \rightarrow 0} \sup _{x \in K} \mathbf{E}_{x}\left[\mathbf{E}_{X_{s}}\left[A_{t}^{2 p}\right]\right]^{1 / 2} \sup _{x \in K} \mathbf{P}_{x}\left(\tau_{G_{n}} \leq s<\zeta\right)+\lim _{t \rightarrow 0} \sup _{x \in G_{n}} \mathbf{E}_{x}\left[A_{t}^{p}\right]
\end{aligned}
$$




$$
\leq \lim _{t \rightarrow 0} \sup _{x \in E} \mathbf{E}_{x}\left[A_{t}^{2 p}\right]^{1 / 2} \sup _{x \in K} \mathbf{P}_{x}\left(\tau_{G_{n}} \leq s<\zeta\right) .
$$

Here we used the assertion (2.1) for $s=0$. Letting $n \rightarrow \infty$, we obtain (2.1) for general $s>0$.

(2): Put $f(x):=\mathbf{E}_{x}\left[A_{t}^{p} B_{t}^{q}\right]$. We only prove for the case $p, q \geq 1$. The proof for other cases are similar. We see $P_{s} f \in C_{b}(E)$ for $s>0$. Take $\left.\left.s \in\right] 0, t\right]$. Since $\left|a^{p}-b^{p}\right| \leq$ $p \max \left\{a^{p-1}, b^{p-1}\right\}|a-b|$ for $a, b \geq 0$, we have

$$
\begin{aligned}
\left|P_{s} f(x)-f(x)\right|= & \left|\mathbf{E}_{x}\left[\mathbf{E}_{X_{s}}\left[A_{t}^{p} B_{t}^{q}\right]\right]-\mathbf{E}_{x}\left[A_{t}^{p} B_{t}^{q}\right]\right| \\
= & \left|\mathbf{E}_{x}\left[\left(A_{t+s}-A_{s}\right)^{p}\left(B_{t+s}-B_{s}\right)^{q} g\left(X_{t+s}\right)-A_{t}^{p} B_{t}^{q}\right]\right| \\
\leq & \left.\mid \mathbf{E}_{x}\left[\left(\left(A_{t+s}-A_{s}\right)^{p}-A_{t}^{p}\right)\left(B_{t+s}-B_{s}\right)^{q}\right)\right] \mid \\
& +\left|\mathbf{E}_{x}\left[A_{t}^{p}\left(\left(B_{t+s}-B_{s}\right)^{q}-B_{t}^{q}\right)\right]\right| \\
\leq & p \mathbf{E}_{x}\left[\left\{\left(A_{t+s}-A_{s}\right)^{p-1} \vee A_{t}^{p-1}\right\}\left|A_{t+s}-A_{s}-A_{t}\right|\left(B_{t+s}-B_{s}\right)^{q}\right] \\
& +q \mathbf{E}_{x}\left[\left\{\left(B_{t+s}-B_{s}\right)^{q-1} \vee B_{t}^{q-1}\right\}\left|B_{t+s}-B_{s}-B_{t}\right| A_{t}^{p}\right] \\
\leq & p \mathbf{E}_{x}\left[A_{2 t}^{p-1} B_{2 t}^{q}\left|A_{t+s}-A_{s}-A_{t}\right|\right]+q \mathbf{E}_{x}\left[B_{2 t}^{q-1}\left|B_{t+s}-B_{s}-B_{t}\right| A_{t}^{p}\right],
\end{aligned}
$$

which uniformly converges to 0 on any compact set $K$, because

$$
\begin{aligned}
\sup _{x \in K} \mathbf{E}_{x}\left[\left|A_{t+s}-A_{s}-A_{t}\right|^{3}\right] & \leq 2^{2} \sup _{x \in K} \mathbf{E}_{x}\left[\left|A_{t+s}-A_{t}\right|^{3}+A_{s}^{3}\right] \\
& \left.\leq 4 \sup _{x \in K} \mathbf{E}_{x}\left[A_{s}^{3} \circ \theta_{t}\right]\right]+4 \sup _{x \in K} \mathbf{E}_{x}\left[A_{s}^{3}\right] \rightarrow 0 \text { as } s \rightarrow 0
\end{aligned}
$$

and $\sup _{x \in E} \mathbf{E}_{x}\left[A_{2 t}^{3(p-1)}\right]<\infty, \sup _{x \in E} \mathbf{E}_{x}\left[B_{2 t}^{3 q}\right]<\infty$. Therefore $f(x)$ is continuous.

(3): From (2), E. $\left[A_{t}^{n} B_{t}^{k}\right] \in C_{b}(E)$ for $n, k \in \mathbb{N} \cup\{0\}$. So it suffices to show the uniform convergence of

$$
\mathbf{E}_{x}\left[e^{A_{t}-B_{t}}\right]=\mathbf{E}_{x}\left[\sum_{n=0}^{\infty} \frac{\left(A_{t}-B_{t}\right)^{n}}{n !}\right]=\mathbf{E}_{x}\left[\sum_{n=0}^{\infty} \frac{1}{n !} \sum_{k=0}^{n}(-1)^{n-k}{ }_{n} C_{k} A_{t}^{k} B_{t}^{n-k}\right]
$$

for sufficiently small $t>0$. We estimate by Lemma 2.1

$$
\begin{aligned}
\sup _{x \in E} \mathbf{E}_{x}\left[\left|\sum_{n=N}^{\infty} \frac{\left(A_{t}-B_{t}\right)^{n}}{n !}\right|\right] & \leq \sum_{n=N}^{\infty} \frac{\sup _{x \in E} \mathbf{E}_{x}\left[\left|A_{t}-B_{t}\right|^{n}\right]}{n !} \\
& \leq \sum_{n=N}^{\infty}\left(\sup _{x \in E} \mathbf{E}_{x}\left[\left|A_{t}-B_{t}\right|\right]\right)^{n} \rightarrow 0 \text { as } N \rightarrow \infty,
\end{aligned}
$$

provided $\sup _{x \in E} \mathbf{E}_{x}\left[\left|A_{t}-B_{t}\right|\right]<1$.

3. Properties on the Feynman-Kac semigroup. In this section, we investigate several properties on $\left(Q_{t}\right)_{t>0}$. We assume that $\mathbf{X}$ has doubly Feller property.

Take a bounded function $v$ defined on $E_{\partial}$ such that $v \in \dot{\mathcal{F}}_{\text {loc }}$. Since $e^{z}-z-1 \leq \frac{1}{2} e^{|z|} z^{2}$,

$$
\left|e^{v(x)-v(y)}-(v(x)-v(y))-1\right| \leq \frac{1}{2} e^{2\|v\|_{\infty}}|v(x)-v(y)|^{2} .
$$


Put $M_{t}:=\int_{0}^{t} e^{v\left(X_{s-}\right)} \mathrm{d} M_{s}^{e^{-v}-1}$ and let

$$
Y_{t}:=\operatorname{Exp}(M)_{t}, \quad t<\zeta
$$

be the solution of the $\operatorname{SDE} Y_{t}=1+\int_{0}^{t} Y_{s-} \mathrm{d} M_{s}, t<\zeta$ relative to $M_{t}$. Here $M^{e^{-v}-1}$ be the locally square integrable MAF on $\llbracket 0, \zeta \llbracket$ appeared in the generalized Fukushima decomposition (Theorem 6.1 below) for $e^{-v}-1 \in \dot{\mathcal{F}}_{\text {loc }} \cap L^{\infty}(E ; m)$. Note that $Y_{t}$ is a positive local martingale on $\llbracket 0, \zeta \llbracket$. Let us denote by $\mathbf{Y}=\left(\Omega, \mathcal{F}_{\infty}^{Y}, \mathcal{F}_{t}^{Y}, X_{t}, \mathbf{P}_{x}^{Y}, \zeta\right)$ the transformed process of $\mathbf{X}$ by $Y_{t}$. The transition semigroup $\left(P_{t}^{Y}\right)_{t>0}$ of $\mathbf{Y}$ is defined by

$$
P_{t}^{Y} f(x):=\mathbf{E}_{x}^{Y}\left[f\left(X_{t}\right)\right]=\mathbf{E}_{x}\left[Y_{t} f\left(X_{t}\right)\right] .
$$

Given a path $\omega \in\{t<\zeta\}$, define a time-reversal operator $r_{t}$ by $r_{t}(\omega)(s)=\omega(t-s)_{-}$for $0 \leq s<t$, and $r_{t}(\omega)(s)=\omega(0)$ for $s \geq t$. A continuous additive functional $C_{t}$ is said to be even if $C_{t} \circ r_{t}=C_{t}$ for every $t<\zeta$. The following lemma is deduced similarly to [10, Lemma 3.2].

LEMMA 3.1. (1) $Y_{t}$ can be represented as follows:

$$
Y_{t}=\exp \left(-M_{t}^{v}-A_{t}^{\mu_{v}}\right), \quad t<\zeta,
$$

where

$$
A_{t}^{\mu_{v}}:=\int_{0}^{t} N\left(e^{v-v(\cdot)}-(v-v(\cdot))-1\right)\left(X_{s}\right) \mathrm{d} H_{s}+\frac{1}{2}\left\langle M^{v, c}\right\rangle_{t} .
$$

(2) $\mathbf{Y}$ is an $e^{-2 v} m$-symmetric Hunt process on $E$.

REMARK 3.1. Note here that $u \in \mathcal{F}_{\text {loc }} \cap C\left(E_{\partial}\right)$ with $\mu_{\langle u\rangle} \in S_{K}^{1}(\mathbf{X})$ admits a generalized Fukushima decomposition in the strict sense by Theorem 6.2(2) below. Then this implies that $\mathbf{E}_{x}\left[Y_{t}\right]=1$ for any $t>0$ and $x \in E$, that is, $Y_{t}$ is a martingale multiplicative functional. Its proof can be done in a way similar to that in [10, Lemma 4.1(ii)]. In this case, $\mathbf{Y}$ satisfies $\mathbf{E}_{x}^{Y}\left[f\left(X_{t}\right)\right]=\mathbf{E}_{x}\left[Y_{t} f\left(X_{t}\right)\right]$ for any $f \in \mathcal{B}_{b}\left(E_{\partial}\right), x \in E_{\partial}$ and $t \geq 0$. Indeed, $\mathbf{Y}$ is constructed on $E$ through the transition probability $P_{t}^{Y}(x, \mathrm{~d} y)$ defined by $P_{t}^{Y} f(x):=\mathbf{E}_{x}\left[Y_{t} f\left(X_{t}\right): t<\zeta\right], x \in E, t>0$ and $f \in \mathcal{B}_{b}(E)$, and is extended to $E_{\partial}$ by adding $\partial$ as a trap point. The transition probability $P_{t}^{Y, \partial}(x, \mathrm{~d} y)$ on $E_{\partial}$ of $\mathbf{Y}$ is given by $P_{t}^{Y, \partial} f(x):=\mathbf{E}_{x}^{Y}\left[f\left(X_{t}\right)\right]=P_{t}^{Y} \mathbf{1}_{E} f(x)+f(\partial)\left(\mathbf{1}_{E_{\partial}}(x)-P_{t}^{Y} \mathbf{1}_{E}(x)\right), x \in E_{\partial}, t>0$ and $f \in \mathcal{B}_{b}\left(E_{\partial}\right)$, satisfies $P_{t}^{Y, \partial} f(x)=\mathbf{E}_{x}\left[Y_{t} f\left(X_{t}\right)\right]$ for $x \in E_{\partial}$ from the martingale property of $Y_{t}^{u}$.

The next lemma is needed for the large deviation principle for our Feynman-Kac semigroups.

LEMma 3.2. Suppose $\mu_{\langle u\rangle} \in S_{K}^{1}(\mathbf{X})$ for $u \in \mathcal{F}_{\text {loc }} \cap C\left(E_{\partial}\right), \mu=\mu_{1}-\mu_{2}$ and $F=F_{1}-F_{2}$ with $\mu_{1}+N\left(F_{1}\right) \mu_{H} \in S_{L K}^{1}(\mathbf{X}) \cap S_{E K}^{1}(\mathbf{X}), \mu_{2}+N\left(F_{2}\right) \mu_{H} \in S_{L K}^{1}(\mathbf{X})$.

(1) Both the semigroups $\left(P_{t}^{Y}\right)_{t>0}$ and $\left(Q_{t}\right)_{t>0}$ have the doubly Feller property. In particular, $\mathbf{Y}$ is a doubly Feller process. 
(2) There exist $p>1, C_{p}\left(=C_{p}\left(u, \mu_{1}, F_{1}\right)\right)>1$ and $\beta_{p}\left(=\beta_{p}\left(u, \mu_{1}, F_{1}\right)\right)>0$ such that

$$
\sup _{x \in E} \mathbf{E}_{x}\left[\left(e_{A}(t)\right)^{p}\right] \leq C_{p} e^{\beta_{p} t}
$$

Hence,

$$
\sup _{x \in E} Q_{t}^{\partial} \mathbf{1}_{E_{\partial}}(x) \leq C e^{\beta t},
$$

where $C:=C_{p}^{1 / p}$ and $\beta:=\beta_{p} / p$, in particular, for any $\alpha>\beta$, we have

$$
\sup _{x \in E} S_{\alpha}^{\partial} \mathbf{1}_{E_{\partial}}(x) \leq \frac{C}{\alpha-\beta} .
$$

(3) Assume further $\mu_{2}+N\left(F_{2}\right) \mu_{H} \in S_{L K}^{1}(\mathbf{X}) \cap S_{D}^{1}(\mathbf{X})$. For each $\alpha>0$, there exists a constant $C>1$ and $\beta>0$ such that

$$
\inf _{x \in E} S_{\alpha}^{\partial} \mathbf{1}_{E_{\partial}}(x) \geq \frac{1}{C(\alpha+\beta)}>0 .
$$

PROOF. (1): By (3.2), $\exp \left(-M_{t}^{u}\right)$ can be expressed as

$$
\exp \left(-M_{t}^{u}\right)=Y_{t} \exp \left(A_{t}^{\mu_{u}}\right) \text {. }
$$

Here $Y_{t}:=Y_{t}^{u}$ for $u \in \mathcal{F}_{\text {loc }} \cap C\left(E_{\partial}\right)$. Since $\mu_{u}(\mathrm{~d} x):=N\left(e^{u(x)-u(\cdot)}-(u(x)-u(\cdot))-\right.$ 1) $(x) \mu_{H}(\mathrm{~d} x)+\frac{1}{2} \mu_{\left\langle M^{u, c}\right\rangle}(\mathrm{d} x) \in S_{K}^{1}(\mathbf{X})$ in view of (3.1), $A^{\mu_{u}}$ corresponding to $\mu_{u}$ is the PCAF in the strict sense. Let $D$ be a relatively compact open set of $E$ and $\mathbf{X}_{D}$ the part process of $\mathbf{X}$ on $D$. Applying [8, Lemmas 2.4(iii) and 3.2(iii)] to $\mathbf{X}_{D}$, we see from the boundedness of $u$ and $F$ that $U_{t}^{(1)}:=\operatorname{Exp}\left(A^{-\mu_{2},-F_{2}}\right)_{t}, U_{t}^{(2)}:=\operatorname{Exp}\left(A^{\mu_{1}, F_{1}}+A^{\mu_{u}}\right)_{t}$ and $U_{t}^{(3)}:=Y_{t}$ satisfy the following; for each $i=1,2,3$ and for any $p \in[1, \infty[$,

$$
\lim _{t \rightarrow 0} \sup _{x \in D} \mathbf{E}\left[\sup _{s \in[0, t]}\left|U_{s}^{(i)}-1\right|^{p}: t<\tau_{D}\right]=0,
$$

where $\tau_{D}:=\inf \left\{t>0: X_{t} \notin D\right\}$ is the first exit time of $X_{t}$ from $D$. By way of the Hölder inequality and $|a+b|^{p} \leq 2^{p-1}\left(|a|^{p}+|b|^{p}\right)$ for $a, b \in \mathbb{R}$, we can easily check that $U_{t}:=\prod_{i=1}^{3} U_{t}^{(i)}=\exp \left(-M_{t}^{u}\right) \operatorname{Exp}\left(A^{\mu, F}\right)_{t}$ satisfies $e_{A}(t)=e^{u\left(X_{t}\right)-u\left(X_{0}\right)} U_{t}$ and

$$
\lim _{t \rightarrow 0} \sup _{x \in D} \mathbf{E}\left[\sup _{s \in[0, t]}\left|U_{s}-1\right|^{p}: t<\tau_{D}\right]=0 .
$$

In particular, we obtain the condition [8, (1.7)] for $B=E$. Since $\mu_{1}+N\left(F_{1}\right) \mu_{H} \in S_{L K}^{1}(\mathbf{X}) \cap$ $S_{E K}^{1}(\mathbf{X})$ and $\mu_{u} \in S_{K}^{1}(\mathbf{X})$, [8, Theorem 2.5] shows that $U_{t}^{(2)}$ satisfies that there exists $p>1$ such that for all $t>0$

$$
\sup _{s \in[0, t]} \sup _{x \in E} \mathbf{E}_{x}\left[\left|U_{s}^{(2)}\right|^{p}: s<\zeta\right]<\infty .
$$

By way of Hölder inequality, we then see that for any $q \in] 1, p[$,

$$
\mathbf{E}_{x}\left[\left|U_{s}\right|^{q}: s<\zeta\right] \leq \mathbf{E}_{x}\left[\left|U_{s}^{(2)}\right|^{p}: s<\zeta\right]^{\frac{q}{p}} \mathbf{E}_{x}\left[\left|U_{s}^{(3)}\right|^{\frac{p q}{p-q}}: s<\zeta\right]^{\frac{p-q}{p q}},
$$


which yields that $U_{t}$ satisfies the condition [8, (1.12)] for $B=E$. Note that the condition $\mu_{\langle u\rangle} \in S_{K}^{1}(\mathbf{X})$ is used for that $Y_{t}=U_{t}^{(3)}$ satisfies [8, (1.7) and (1.12)] with any $p>1$ for $B=E$ by way of a corrected version of Lemma 3.2(ii) in [8]. Hence Corollary 1.5 in [8] tells us that $\left(P_{t}^{Y}\right)_{t>0}$ has doubly Feller property and the semigroup $\left(P_{t}^{u, \mu, F}\right)_{t>0}$ defined by

$$
P_{t}^{u, \mu, F} f(x):=\mathbf{E}_{x}\left[e^{-M_{t}^{u}} \operatorname{Exp}\left(A^{\mu, F}\right)_{t} f\left(X_{t}\right)\right] \quad \text { for } f \in \mathcal{B}_{b}(E)
$$

also has doubly Feller property. Therefore $\left(Q_{t}\right)_{t>0}$ has the desired doubly Feller property because $Q_{t} f(x)=e^{-u(x)} P_{t}^{u, \mu, F}\left(f e^{u}\right)(x)$ for all $x \in E$.

(2): Let $Y_{t}$ and $A_{t}^{\mu_{u}}$ be as above. Note that there exist $p>1$ and $q>1$ being close to 1 with $p q \in] 1,2[$ such that

$$
\left(p q+(p q-1)\left\|F_{1}\right\|_{\infty}\right) v_{\mu_{u}, \mu_{1}, F_{1}}
$$

is a measure of extended Kato class, where $v_{\mu_{u}, \mu_{1}, F_{1}}:=\mu_{u}+\mu_{1}+N\left(F_{1}\right) \mu_{H}$. For such $p>1, q>1, A^{v_{p q \mu_{u}} p q \mu_{1},\left(1+F_{1}\right)^{p q}-1}$ is a PAF of extended Kato class in view of the inequality $(1+x)^{p q}-1 \leq p q x+(p q-1) x^{2}$ for $x>-1$. Therefore, we have from (3.2) and [44, Lemma 2.1(b)] that

$$
\begin{aligned}
& \mathbf{E}_{x}\left[e_{A}(t)^{p}\right]=\mathbf{E}_{x}\left[e^{p\left(u\left(X_{t}\right)-u\left(X_{0}\right)-M_{t}^{u}\right)} \operatorname{Exp}\left(A^{\mu, F}\right)_{t}^{p}\right] \\
& \leq e^{2 p\|u\|_{\infty}} \mathbf{E}_{x}\left[e^{-p M_{t}^{u}} e^{p A_{t}^{\mu_{1}}} \operatorname{Exp}\left(A^{F_{1}}\right)_{t}^{p}\right] \\
& =e^{2 p\|u\|_{\infty}} \mathbf{E}_{x}\left[Y_{t}^{p} e^{p A_{t}^{\mu_{u}}+p A_{t}^{\mu_{1}}} \operatorname{Exp}\left(A^{F_{1}}\right)_{t}^{p}\right] \\
& \leq e^{2 p\|u\|_{\infty}} \mathbf{E}_{x}\left[Y_{t}^{\frac{p q}{q-1}}\right]^{\frac{q-1}{q}} \mathbf{E}_{x}\left[e^{p q A_{t}^{\mu_{u}}+p q A_{t}^{\mu_{1}}} \operatorname{Exp}\left(A^{F_{1}}\right)_{t}^{p q}\right]^{\frac{1}{q}} \\
& =e^{2 p\|u\|_{\infty}} \mathbf{E}_{x}\left[Y_{t}^{\frac{p q}{q-1}}\right]^{\frac{q-1}{q}} \mathbf{E}_{x}\left[\operatorname{Exp}\left(A^{\left.v_{p q \mu_{u}, p q \mu_{1},\left(1+F_{1}\right) p q}\right)^{p}}\right)_{t}\right]^{\frac{1}{q}} \\
& \leq e^{2 p\|u\|_{\infty}} c_{p, q}^{(1)}(u) c_{p, q}^{(2)}\left(u, \mu_{1}, F_{1}\right) e^{\beta_{p, q}\left(u, \mu_{1}, F_{1}\right) t} .
\end{aligned}
$$

Note that all coefficients are greater than 1 , because of $\mathbf{E}_{x}\left[Y_{t}^{\frac{p q}{q-1}}\right] \geq \mathbf{E}_{x}\left[Y_{t}\right]^{\frac{p q}{q-1}}=1$ and $c_{p, q}^{(2)}\left(u, \mu_{1}, F_{1}\right)>1$. So we may assume that these are strictly greater than 1 .

(3): Set $F_{2}^{(1)}:=\frac{F_{2}}{1-F_{2}} \leq \frac{F_{2}}{1-\left\|F_{2}\right\|_{\infty}}$. We see $\mu_{2}+N\left(F_{2}^{(1)}\right) \mu_{H} \in S_{L K}^{1}(\mathbf{X}) \cap S_{D}^{1}(\mathbf{X})$. Let $\delta \in] 0,1\left[\right.$ be such that $\delta\left(\mu_{2}+N\left(F_{2}^{(1)}\right) \mu_{H}\right) \in S_{L K}^{1}(\mathbf{X}) \cap S_{E K}^{1}(\mathbf{X})$. In a similar way of the proof of (2) with $\delta \mu_{-u}, \delta \mu_{2}$ and $\delta F_{2}^{(1)}$, there exist $p>1$ and $q>1$ close to 1 with $\left.p q \delta \in\right] 0,1[$ such that $A^{v} p q \delta \mu_{-u}, p q \delta \mu_{2},\left(1+F_{2}^{(1)}\right)^{p q \delta} \delta_{-1}$ is also a PAF of extended Kato class in view of the inequality $(1+x)^{p q \delta}-1 \leq p q \delta x$ for $x>-1$. So we have

$$
\begin{aligned}
& \mathbf{E}_{x}\left[e_{A}(t)^{-p \delta}\right]=\mathbf{E}_{x}\left[e^{p \delta N_{t}^{-u}-p \delta A_{t}^{\mu}} \operatorname{Exp}\left(A^{F}\right)_{t}^{-p \delta}\right] \\
& \leq e^{2 p \delta\|u\|_{\infty}} \mathbf{E}_{x}\left[e^{-p \delta M_{t}^{-u}} e^{p \delta A_{t}^{\mu_{2}}} \operatorname{Exp}\left(A^{-F_{2}}\right)_{t}^{-p \delta}\right] \\
& \leq e^{2 p \delta\|u\|_{\infty}} c_{p, q, \delta}^{(1)}(-u) \mathbf{E}_{x}\left[\operatorname{Exp}\left(A^{\left.v_{p q \delta \mu_{-u}, p q \delta \mu_{2},\left(1-F_{2}\right.}\right)^{-p q \delta}-1}\right)_{t}\right]^{\frac{1}{q}}
\end{aligned}
$$

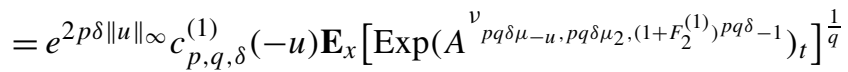

$$
\begin{aligned}
& \leq e^{2 p\|u\|_{\infty}} c_{p, q, \delta}^{(1)}(-u) c_{p, q, \delta}^{(2)}\left(-u, \mu_{2}, F_{2}\right) e^{\beta_{p, q, \delta}\left(-u, \mu_{2}, F_{2}\right) t} .
\end{aligned}
$$


From this,

$$
\begin{aligned}
1 & =\mathbf{E}_{x}\left[\mathbf{1}_{E_{\partial}}\right]^{2}=\mathbf{E}_{x}\left[e_{A}(t)^{\frac{\delta}{2}} e_{A}(t)^{-\frac{\delta}{2}}\right]^{2} \\
& \leq \mathbf{E}_{x}\left[e_{A}(t)^{\delta}\right] \mathbf{E}_{x}\left[e_{A}(t)^{-p \delta}\right]^{\frac{1}{p}} \leq \mathbf{E}_{x}\left[e_{A}(t)^{\delta}\right] C e^{\beta t}
\end{aligned}
$$

for some $C=C_{p, q, \delta}\left(-u, \mu_{2}, F_{2}\right)>1$ and $\beta=\beta_{p, q, \delta}\left(-u, \mu_{2}, F_{2}\right)>0$. Hence

$$
\mathbf{E}_{x}\left[e_{A}(t)\right]^{\delta} \geq \mathbf{E}_{x}\left[e_{A}(t)^{\delta}\right] \geq C^{-1} e^{-\beta t}
$$

for all $x \in E$. Finally, the relation $\mathbf{E}_{x}\left[e_{A}(t)\right] \geq C^{-1 / \delta} e^{-(1 / \delta) \beta t}$ implies the assertion.

REMARK 3.2. In the same way as in the proof of Lemma 3.2(1), we can prove the following. If $\mu_{\langle u\rangle} \in S_{K}^{1}(\mathbf{X}), \mu=\mu_{1}-\mu_{2} \in S_{K}^{1}(\mathbf{X})-S_{K}^{1}(\mathbf{X})$ and $F=F_{1}-F_{2} \in J_{K}^{1}(\mathbf{X})-$ $J_{K}^{1}(\mathbf{X})$, then we can obtain that $\left(Q_{t}\right)_{t>0}$ satisfies the conditions (a) and (c) in [11] (see (1.3), (1.4) and (1.6) in [8]). So the proof of Theorem 2 in [11] yields the strong Feller property of $\left(Q_{t}\right)_{t>0}$ without assuming the Feller property of $\mathbf{X}$ (see also Theorem 1.1, Corollary 1.2 and Remark 1.3 in [8]).

Let $C_{u}(E)$ be the set of continuous functions on $E$ that has the limit as $x \rightarrow \partial$. In other words, each element in $C_{u}(E)$ is the restriction of some function in $C\left(E_{\partial}\right)$. For $f \in C_{u}(E)$, we write $f(\partial):=\lim _{x \rightarrow \partial} f(x)$. Let $\operatorname{USC}\left(E_{\partial}\right)$ be the family of all upper semi continuous functions on $E_{\partial}$ and $U S C_{u}(E)$ a family of functions on $E$ whose element is the restriction of some function in $\operatorname{USC}\left(E_{\partial}\right)$. We set $C_{u}^{+}(E):=\left\{f \in C_{u}(E) \mid f \geq 0\right.$ on $\left.E\right\}$ and $U S C_{u}^{+}(E):=$ $\left\{f \in U S C_{u}(E) \mid f \geq 0\right.$ on $\left.E\right\}$.

The main theorem of this section is the following:

THEOREM 3.1. Suppose $\mu_{\langle u\rangle} \in S_{K_{\infty}^{+}}^{1}(\mathbf{X}), \mu=\mu_{1}-\mu_{2} \in S_{K_{\infty}^{+}}^{1}(\mathbf{X})-S_{L K}^{1}(\mathbf{X})$ and $F=F_{1}-F_{2} \in J_{K_{\infty}^{+}}^{1}(\mathbf{X})-J_{L K}^{1}(\mathbf{X})$. Then $Q_{t}\left(U S C_{u}^{+}(E)\right) \subset U S C_{u}^{+}(E)$ for each $t>0$. If further $\mu_{2}+N\left(F_{2}\right) \mu_{H} \in S_{K_{\infty}^{+}}^{1}(\mathbf{X})$, then $Q_{t}\left(C_{u}(E)\right) \subset C_{u}(E)$ for each $t>0$.

To prove this theorem, we need several lemmas below. When $u=0$, we write $P_{t}^{\mu, F}$ (resp. $R_{\alpha}^{\mu, F}$ ) instead of $Q_{t}$ (resp. $S_{\alpha}$ ). The following lemma is a slight extension of Theorem 2.1(iv) of [36] without assuming the transience and conservativeness of $\mathbf{X}$, and also generalizes Lemma 3.2 in [12]. The proof of Lemma 3.2 in [12] has a gap derived from the incorrect statement of Lemma A.1 in [12]. The proof of Lemma 3.3 below fulfills the gap.

Lemma 3.3. Suppose $\mu=\mu_{1}-\mu_{2} \in S_{K_{\infty}^{+}}^{1}(\mathbf{X})-S_{L K}^{1}(\mathbf{X})$ and $F=F_{1}-F_{2} \in$ $J_{K_{\infty}^{+}}^{1}(\mathbf{X})-J_{L K}^{1}(\mathbf{X})$. Then $P_{t}^{\mu, F}\left(U S C_{u}^{+}(E)\right) \subset U S C_{u}^{+}(E)$ for each $t>0$. If further $\mu_{2}+$ $N\left(F_{2}\right) \mu_{H} \in S_{K_{\infty}^{+}}^{1}(\mathbf{X})$, then $P_{t}^{\mu, F}\left(C_{u}(E)\right) \subset C_{u}(E)$ for each $t>0$.

Proof. First we assume $\mu_{2}+N\left(F_{2}\right) \mu_{H} \in S_{K_{\infty}^{+}}^{1}(\mathbf{X})$. We use the convention $P_{t}^{\mu, F, \partial} f(x)$ $:=\mathbf{E}_{x}\left[\operatorname{Exp}\left(A^{\mu, F}\right)_{t} f\left(X_{t}\right)\right]$ for $f \in \mathcal{B}\left(E_{\partial}\right)$. For any $f \in C_{u}^{+}(E), f-f(\partial) \mathbf{1}_{E_{\partial}} \in C_{\infty}(E)$. So for proving $P_{t}^{\mu, F} f=P_{t}^{\mu, F}\left(f-f(\partial) \mathbf{1}_{E_{\partial}}\right)+f(\partial) P_{t}^{\mu, F, \partial} \mathbf{1}_{E_{\partial}} \in C_{u}^{+}(E)$, we first need to show $P_{t}^{\mu, F, \partial} \mathbf{1}_{E_{\partial}} \in C(E)$. This holds for sufficiently small $t>0$ by Proposition 2.1(3). 
Next we prove the continuity of $P_{t}^{\mu, F, \partial} \mathbf{1}_{E_{\partial}}$ at $\partial$. Since $P_{t}^{\mu, F, \partial} \mathbf{1}_{E_{\partial}}(\partial)=1$, it suffices to show

$$
\lim _{x \rightarrow \partial} P_{t}^{\mu, F, \partial} \mathbf{1}_{E_{\partial}}(x)=\lim _{x \rightarrow \partial} \mathbf{E}_{x}\left[\operatorname{Exp}\left(A^{\mu, F}\right)_{t}\right]=1 .
$$

Let $v \in S_{K_{\infty}^{+}}^{1}(\mathbf{X}), U \in J_{K_{\infty}^{+}}^{1}(\mathbf{X})$ with $M \geq U \geq 0$ on $E \times E$ for some $M>0$. Note that $V:=e^{U}-1 \in J_{K_{\infty}^{+}}^{1}(\mathbf{X})$ because of the boundedness of the function $x \mapsto x^{-1}\left(e^{x}-1\right)$ on $[0, M]$. Put $\mathbf{1}_{B} V(x, y):=\mathbf{1}_{B}(x) V(x, y), x, y \in E$ for $B \subset E$. In the same way as in the proof after pp. 704 line -12 in [12, Lemma 3.2], we can get

$$
\lim _{x \rightarrow \partial} \mathbf{E}_{x}\left[\operatorname{Exp}\left(A^{2 \mathbf{1}_{K} v, 2 \mathbf{1}_{K} V}\right)_{t}\right]=1
$$

for any compact set $K$ of $E$. Moreover,

$$
\lim _{\substack{K: c o m p a c t \\ K \uparrow E}} \sup _{x \in E} \mathbf{E}_{x}\left[\operatorname{Exp}\left(A^{2 \mathbf{1}_{K^{c}} v, 2 \mathbf{1}_{K^{c}} V}\right)_{t}\right] \leq 1
$$

by Khasminskii's inequality for Stieltjes exponential (Lemma 2.1(a) in [44]). Indeed, owing to the property of Lévy system,

$$
\mathbf{E}_{x}\left[A_{t}^{2 \mathbf{1}_{K^{c}} v, 2 \mathbf{1}_{K^{c}} V}\right]=\mathbf{E}_{x}\left[A_{t}^{2 \mathbf{1}_{K^{c}}\left(v+N(V) \mu_{H}\right)}\right]
$$

for all $x \in E$ and by the assumptions of $v$ and $U$, we see

$$
\lim _{\substack{K: \text { compact } \\ K \uparrow E}} \sup _{x \in E} \mathbf{E}_{x}\left[A_{t}^{2 \mathbf{1}_{K^{c} v}, 2 \mathbf{1}_{K^{c}} V}\right]=0 .
$$

Therefore

$$
\begin{aligned}
\varlimsup_{x \rightarrow \partial} \mathbf{E}_{x}\left[\operatorname{Exp}\left(A^{v, V}\right)_{t}\right] & =\varlimsup_{x \rightarrow \partial} \mathbf{E}_{x}\left[\operatorname{Exp}\left(A^{\mathbf{1}_{K} v \mathbf{1}_{K} V}\right)_{t} \operatorname{Exp}\left(A^{\mathbf{1}^{c} v, \mathbf{1}_{K} V}\right)_{t}\right] \\
& \leq \varlimsup_{x \rightarrow \partial} \mathbf{E}_{x}\left[\operatorname{Exp}\left(A^{2 \mathbf{1}_{K} v, 2 \mathbf{1}_{K} V}\right)_{t}\right]^{1 / 2} \mathbf{E}_{x}\left[\operatorname{Exp}\left(A^{2 \mathbf{1}_{K^{c}} v, 2 \mathbf{1}_{K^{c}} V}\right)_{t}\right]^{1 / 2} \\
& \leq \sup _{x \in E} \mathbf{E}_{x}\left[\operatorname{Exp}\left(A^{2 \mathbf{1}_{K^{c}} v, 2 \mathbf{1}_{K} V}\right)_{t}\right]^{1 / 2} \downarrow 1
\end{aligned}
$$

as $K \uparrow E$ with $K$ being compact, and thus

$$
\varliminf_{x \rightarrow \partial} \mathbf{E}_{x}\left[\operatorname{Exp}\left(A^{v, V}\right)_{t}^{-1}\right] \geq \frac{1}{\overline{\lim }_{x \rightarrow \partial} \mathbf{E}_{x}\left[\operatorname{Exp}\left(A^{v, V}\right)_{t}\right]} \geq 1 .
$$

Now we have

$$
\varlimsup_{x \rightarrow \partial} \mathbf{E}_{x}\left[\operatorname{Exp}\left(A^{v, V}\right)_{t}\right] \leq 1 \leq \varliminf_{x \rightarrow \partial} \mathbf{E}_{x}\left[\operatorname{Exp}\left(A^{v, V}\right)_{t}^{-1}\right] .
$$

Noting that for $\mu=\mu_{1}-\mu_{2} \in S_{K_{\infty}^{+}}^{1}(\mathbf{X})-S_{K_{\infty}^{+}}^{1}(\mathbf{X})$ and $F=F_{1}-F_{2} \in J_{K_{\infty}^{+}}^{1}(\mathbf{X})-J_{K_{\infty}^{+}}^{1}(\mathbf{X})$ we have

$$
\mathbf{E}_{x}\left[\operatorname{Exp}\left(A^{\mu_{2}, F_{2}^{(1)}}\right)_{t}^{-1}\right] \leq \mathbf{E}_{x}\left[\operatorname{Exp}\left(A^{\mu, F}\right)_{t}\right] \leq \mathbf{E}_{x}\left[\operatorname{Exp}\left(A^{\mu_{1}, F_{1}}\right)_{t}\right]
$$

for $F_{2}^{(1)}:=F_{2} /\left(1-F_{2}\right)$ because $\operatorname{Exp}\left(A^{-F_{2}}\right)_{t}=\operatorname{Exp}\left(A^{F_{2}^{(1)}}\right)_{t}^{-1}$. Since $\left\|_{1-F_{2}}\right\|_{\infty} \leq \frac{1}{1-\left\|F_{2}\right\|_{\infty}}$ $<\infty$, we see $F_{2}^{(1)} \in J_{K_{\infty}^{+}}^{1}(\mathbf{X})$, and thus

$$
\lim _{x \rightarrow \partial} \mathbf{E}_{x}\left[\operatorname{Exp}\left(A^{\mu, F}\right)_{t}\right]=1 .
$$


Therefore, we have $P_{t}^{\mu, F}\left(C_{u}^{+}(E)\right) \subset C_{u}^{+}(E)$ for sufficiently small $t>0$. By the semigroup property of $\left(P_{t}^{\mu, F}\right)_{t>0}$, we obtain the assertion for any $t>0$.

Next we only assume $\mu_{2} \in S_{L K}^{1}(\mathbf{X})$ and $F_{2} \in J_{L K}^{1}(\mathbf{X})$. Let $K$ be a compact set. Then $\mathbf{1}_{K} \mu_{2} \in S_{K}^{1}(\mathbf{X})$ and $\mathbf{1}_{K} F_{2} \in J_{K}^{1}(\mathbf{X})$, where $\mathbf{1}_{K} F_{2}(x, y):=\mathbf{1}_{K}(x) F_{2}(x, y), x, y \in E$. Moreover, we have $\mathbf{1}_{K} \mu_{2} \in S_{K_{\infty}^{+}}^{1}(\mathbf{X})$ and $\mathbf{1}_{K} F_{2} \in J_{K_{\infty}^{+}}^{1}(\mathbf{X})$ from the definitions for $S_{K_{\infty}^{+}}^{1}(\mathbf{X})$ and $J_{K_{\infty}^{+}}^{1}(\mathbf{X})$ which implies $P_{t}^{\mu_{K}, F_{K}}\left(C_{u}^{+}(E)\right) \subset C_{u}^{+}(E)$ for any $t>0$, where $\mu_{K}:=\mu_{1}-\mathbf{1}_{K} \mu_{2}$ and $F_{K}:=F_{1}-\mathbf{1}_{K} F_{2}$. Letting $K \uparrow E$, we have $P_{t}^{\mu, F}\left(C_{u}^{+}(E)\right) \subset U S C_{u}^{+}(E)$ for any $t>0$. Since any element of $U S C_{u}^{+}(E)$ is a limit of decreasing sequence from $C_{u}^{+}(E)$ and any limit of decreasing sequence from $\operatorname{USC}_{u}^{+}(E)$ belongs to $U S C_{u}^{+}(E)$, we obtain the desired assertion.

The following lemma can be proved by the same argument as in the proof of [14, Lemma 4.4] in the framework of (quasi-)regular Dirichlet forms (see also the remark before Theorem 2.5 in [5]. Under $\mu_{\langle v\rangle} \in S_{K}(\mathbf{X})$ for $v \in \mathcal{F}_{e}$ see Theorem A.2 in [10].)

LEMma 3.4. Suppose that $v$ is a bounded function defined on $E_{\partial}$ such that $v \in \dot{\mathcal{F}}_{\text {loc. }}$. Let $\mathbf{Y}$ be the transformed process by the multiplicative functional $Y_{t}^{v}$. Let $A^{v}$ be a continuous additive functional of $\mathbf{X}$ with signed Revuz measure $v$. Then the Revuz measure of $A^{v}$ as a continuous additive functional of $\mathbf{Y}$ is $e^{-2 v} v$.

The next theorem can be proved by nearly same arguments as in [10] with Lemma 3.4 above. Its proof depends on the generalized Fukushima decomposition holding up to infinity (see Theorem 6.2(2)).

THEOREM 3.2 (cf. [10]). Suppose that $v$ is a bounded strictly $\mathcal{E}$-quasi continuous function defined on $E_{\partial}$ such that $v \in \dot{\mathcal{F}}_{\text {loc }}$ admits Fukushima's decomposition holding up to infinity under $\mathbf{P}_{x}$-a.s. for q.e. starting point $x \in E$. Let $\left(\mathcal{E}^{Y}, \mathcal{F}^{Y}\right)$ be the Dirichlet form on $L^{2}\left(E ; e^{-2 v} m\right)$ associated with the transformed process $\mathbf{Y}$ by $Y_{t}$. Then $\mathcal{F}^{Y}=\mathcal{F}$ and

$$
\begin{aligned}
\mathcal{E}^{Y}(f, f)= & \frac{1}{2} \int_{E} e^{-2 v(x)} \mu_{\langle f\rangle}^{c}(\mathrm{~d} x)+\iint_{E \times E}(f(x)-f(y))^{2} e^{-v(x)-v(y)} J(\mathrm{~d} x \mathrm{~d} y) \\
& +\int_{E} f(x)^{2} e^{-v(\partial)-v(x)} \kappa(\mathrm{d} x)
\end{aligned}
$$

for any $f \in \mathcal{F}^{Y}=\mathcal{F}$. Moreover, if $\kappa=0$, then the conclusion holds without assuming the Fukushima decomposition holding up to infinity.

Let $J_{1}(\mathbf{Y})$ be the family of functions with respect to $\mathbf{Y}$ as the class $J_{1}(\mathbf{X})$ is defined with respect to $\mathbf{Y}$. The classes $J_{K}^{1}(\mathbf{Y}), J_{L K}^{1}(\mathbf{Y}), J_{E K}^{1}(\mathbf{Y}), J_{K_{\infty}^{+}}^{1}(\mathbf{Y})$ and $J_{K_{\infty}}^{1}(\mathbf{Y})$ are similarly defined for $\mathbf{Y}$ as well as $J_{K}^{1}(\mathbf{X}), J_{L K}^{1}(\mathbf{X}), J_{E K}^{1}(\mathbf{X}), J_{K_{\infty}^{+}}^{1}(\mathbf{X})$ and $J_{K_{\infty}}^{1}(\mathbf{X})$ are defined for $\mathbf{X}$. Using Theorem 3.2, we then have the following:

LEMMA 3.5 (cf. [12, Lemma 3.3], [20, Lemma 4.6]). Let $\mathbf{Y}$ be the transformed process by $Y_{t}:=Y_{t}^{u}$ for $u \in \dot{\mathcal{F}}_{\text {loc }} \cap C\left(E_{\partial}\right)$ with $\mu_{\langle u\rangle} \in S_{K}^{1}(\mathbf{X})$. Then the following hold:

(1) For $v \in S_{D}^{1}(\mathbf{X})\left(\right.$ resp. $\left.v \in S_{00}(\mathbf{X})\right), e^{-2 u} v \in S_{D}^{1}(\mathbf{Y})\left(\right.$ resp. $e^{-2 u} v \in S_{00}(\mathbf{Y})$ ). 
(2) For $v \in S_{1}(\mathbf{X})$ (resp. $\left.F \in J_{1}(\mathbf{X})\right), e^{-2 u} v \in S_{1}(\mathbf{Y})$ (resp. $e^{-2 u} F \in J_{1}(\mathbf{Y})$ ).

(3) For $v \in S_{K}^{1}(\mathbf{X})\left(\right.$ resp. $\left.F \in J_{K}^{1}(\mathbf{X})\right), e^{-2 u} v \in S_{K}^{1}(\mathbf{Y})$ (resp. $\left.e^{-2 u} F \in J_{K}^{1}(\mathbf{Y})\right)$. In particular, $v \in S_{L K}^{1}(\mathbf{X})$ (resp. $F \in J_{L K}^{1}(\mathbf{X})$ ) implies $e^{-2 u} v \in S_{L K}^{1}(\mathbf{Y})$ (resp. $e^{-2 u} F \in$ $\left.J_{L K}^{1}(\mathbf{Y})\right)$.

(4) For $v \in S_{E K}^{1}(\mathbf{X})$ (resp. $\left.F \in J_{E K}^{1}(\mathbf{X})\right), e^{-2 u} v \in S_{E K}^{1}(\mathbf{Y})$ (resp. $e^{-2 u} F \in J_{E K}^{1}(\mathbf{Y})$ ).

(5) For $v \in S_{K_{\infty}^{+}}^{1}(\mathbf{X})$ (resp. $\left.F \in J_{K_{\infty}^{+}}^{1}(\mathbf{X})\right), e^{-2 u} v \in S_{K_{\infty}^{+}}^{1}(\mathbf{Y})$ (resp. $e^{-2 u} F \in J_{K_{\infty}^{+}}^{1}(\mathbf{Y})$ ).

PROOF. The proof is a repetition of the proof of [12, Lemma 3.3]. We omit the details. We only note that under $\mu_{\langle u\rangle} \in S_{K}^{1}(\mathbf{X}), Y_{t}$ is a martingale multiplicative functional defined for all $t \in\left[0, \infty\left[\right.\right.$ and it satisfies $\sup _{x \in E} \mathbf{E}_{x}\left[Y_{t}^{k}\right]<\infty$ for any $k \in \mathbb{N}$.

Now we prove Theorem 3.1.

Proof of Theorem 3.1. Take $f \in U S C_{u}^{+}(E)$. We then see

$$
\begin{aligned}
Q_{t} f(x) & =e^{-u(x)} \mathbf{E}_{x}\left[Y_{t} e^{A_{t}^{\mu_{u}}} \operatorname{Exp}\left(A^{\mu, F}\right)_{t}\left(f e^{u}\right)\left(X_{S}\right)\right] \\
& =e^{-u(x)} \mathbf{E}_{x}^{Y}\left[\operatorname{Exp}\left(A^{\mu_{u}}+A^{\mu, F}\right)_{t}\left(f e^{u}\right)\left(X_{S}\right)\right] .
\end{aligned}
$$

Since $A_{t}^{\mu_{u}}$ and $A_{t}^{\mu_{1}}$ are PCAFs of Kato class in the strict sense under $\mathbf{X}$ whose Revuz measures under $\mathbf{X}$ have positive order Green-tightness, they are also PCAFs of Kato class in the strict sense under $\mathbf{Y}$ whose Revuz measures under $\mathbf{Y}$ have positive order Green-tightness by Lemma 3.5(5). We see that $A_{t}^{\mu_{2}}$ is also a PCAF of local Kato class in the strict sense whose Revuz measure under $\mathbf{Y}$ is of local Kato class by Lemma 3.5(3). Similarly, $A_{t}^{F_{1}}$ (resp. $A_{t}^{F_{2}}$ ) is also PAF in the strict sense under $\mathbf{Y}$ and the Revuz measure of its dual predictable projection under $\mathbf{Y}$ has positive order Green-tightness (resp. is of local Kato class) by Lemma 3.5(5) (resp. Lemma 3.5(3)).

Recall that $\mathbf{Y}$ is a doubly Feller process. We can apply Lemma 3.3 to $\mathbf{Y}$. Therefore $Q_{t} f \in U_{S} C_{u}^{+}(E)$. The latter assertion is similarly proved.

4. Proofs of Theorems 1.1, 1.2, 1.3 and Corollaries 1.1, 1.2. Define the generator $\mathcal{A}$ by

$$
\mathcal{A} \phi=\alpha \phi-g \quad \text { for } \phi=S_{\alpha} g, \quad g \in \mathcal{B}_{b}(E)
$$

Set

$$
\mathcal{D}_{+}(\mathcal{A}):=\left\{\phi=S_{\alpha} g \mid \alpha>\beta, g \in L^{2}(E ; m) \cap C_{b}^{+}(E), g \not \equiv 0\right\},
$$

where $\beta$ is the constant appeared in (3.4). For $\alpha>\beta$ and $\phi=S_{\alpha} g \in \mathcal{D}_{+}(\mathcal{A})$, put

$$
M_{t}^{u, \mu, \phi, F}:=e^{-\alpha t} e_{A}(t) \phi\left(X_{t}\right)-\phi\left(X_{0}\right)-\int_{0}^{t} e^{-\alpha s} e_{A}(s)(\mathcal{A}-\alpha) \phi\left(X_{s}\right) \mathrm{d} s .
$$

Then $M^{u, \mu, \phi, F}$ is a $\mathbf{P}_{x}$-martingale for all $x \in E$ because $\mathbf{E}_{x}\left[M_{t}^{u, \mu, \phi, F}\right]=0$ and

$$
M_{s+t}^{u, \mu, \phi, F}=M_{s}^{u, \mu, \phi, F}+e^{-\alpha s} e_{A}(s) M_{t}^{u, \mu, \phi, F}\left(\theta_{s}\right) \quad \text { for } t, s \geq 0 \quad \mathbf{P}_{x} \text {-a.s. }
$$

for all $x \in E$ hold. Note that $e_{A}(t) e_{-A}(t-)=1+F\left(X_{t-}, X_{t}\right)$. Let denote by $M^{\phi}$ the martingale part of Fukushima's decomposition for $\phi\left(X_{t}\right)-\phi\left(X_{0}\right)$. The next lemma is a slight modification of [12, Lemma 4.1] by applying [19, Lemma 3.1]. 
LEMMA 4.1. $M_{t}^{u, \mu, \phi, F}$ can be written as

$$
M_{t}^{u, \mu, \phi, F}=\int_{0}^{t} e^{-\alpha s} e_{A}(s-) \mathrm{d}\left(M_{s}^{\phi}+M_{s}^{\phi, F}\right),
$$

where

$$
M_{t}^{\phi, F}:=\sum_{0<s \leq t} \phi\left(X_{S}\right) F\left(X_{s-}, X_{S}\right)-\int_{0}^{t} \int_{E} \phi(y) F\left(X_{s}, y\right) N\left(X_{s}, \mathrm{~d} y\right) \mathrm{d} H_{s} .
$$

Let

$$
\check{M}_{t}:=\int_{0}^{t} \frac{1}{\phi\left(X_{s-}\right)} \mathrm{d}\left(M_{s}^{\phi}+M_{s}^{\phi, F}\right), \quad t<\zeta .
$$

The unique solution of Doléans-Dade equation relative to $\check{M}_{t}$,

$$
Z_{t}=1+\int_{0}^{t} Z_{s-} \mathrm{d} \check{M}_{s}, \quad t<\zeta
$$

is then given by

$$
\begin{aligned}
Z_{t}= & \exp \left(\check{M}_{t}-\frac{1}{2}\left\langle\check{M}^{c}\right\rangle_{t}\right) \\
& \times \prod_{0<s \leq t} \frac{\left(1+F\left(X_{s-}, X_{s}\right)\right) \phi\left(X_{s}\right)}{\phi\left(X_{s-}\right)} \exp \left(\frac{\phi\left(X_{s-}\right)-\left(1+F\left(X_{s-}, X_{s}\right)\right) \phi\left(X_{s}\right)}{\phi\left(X_{s-}\right)}\right),
\end{aligned}
$$

where $\left\langle\check{M}^{c}\right\rangle_{t}$ is the quadratic variation of the continuous martingale part of $\check{M}_{t}$. Note that $Z_{t}$ is a positive local martingale on $[0, \zeta[$.

Applying Itô's formula for semi-martingale (with jumps) to $\log \left(e^{-\alpha t} e_{A}(t) \phi\left(X_{t}\right)\right)$ and using Lemma 4.1, we get

$$
\begin{aligned}
\log \left(e^{-\alpha t}\right. & \left.e_{A}(t) \phi\left(X_{t}\right)\right)-\log \left(\phi\left(X_{0}\right)\right) \\
= & \int_{0}^{t} \frac{1}{e^{-\alpha s} e_{A}(s-) \phi\left(X_{s-}\right)} \mathrm{d} M_{s}^{u, \mu, \phi, F}+\int_{0}^{t} \frac{(\mathcal{A}-\alpha) \phi\left(X_{s}\right)}{\phi\left(X_{s}\right)} \mathrm{d} s \\
& -\frac{1}{2} \int_{0}^{t} \frac{1}{e^{-2 \alpha s} e_{A}(s-)^{2} \phi\left(X_{s-}\right)^{2}} \mathrm{~d}\left\langle M^{u, \mu, \phi, F, c}\right\rangle_{s} \\
& +\sum_{0<s \leq t}\left(\log \frac{e_{A}(s) \phi\left(X_{s}\right)}{e_{A}(s-) \phi\left(X_{s-}\right)}-\frac{e_{A}(s) \phi\left(X_{s}\right)-e_{A}(s-) \phi\left(X_{s-}\right)}{e_{A}(s-) \phi\left(X_{s-}\right)}\right) \\
= & \check{M}_{t}+\int_{0}^{t} \frac{(\mathcal{A}-\alpha) \phi\left(X_{s}\right)}{\phi\left(X_{s}\right)} \mathrm{d} s-\frac{1}{2}\left\langle\check{M}^{c}\right\rangle_{t}+\sum_{0<s \leq t}\left(\log \left(1+\Delta \check{M}_{s}\right)-\Delta \check{M}_{s}\right), \quad t<\zeta .
\end{aligned}
$$

Therefore we see that $Z_{t}$ defined in (4.3) can be represented as follows:

$$
Z_{t}=e_{A}(t) \frac{\phi\left(X_{t}\right)}{\phi\left(X_{0}\right)} \exp \left(-\int_{0}^{t} \frac{\mathcal{A} \phi\left(X_{s}\right)}{\phi\left(X_{s}\right)} \mathrm{d} s\right), \quad t<\zeta .
$$

Let us denote by $\mathbf{Z}=\left(X_{t}, \mathbf{P}_{x}^{Z}, x \in E\right)$ the transformed process of $\mathbf{X}$ by the multiplicative functional (4.4). Note that $\phi \in \mathcal{F}$ implies $\int_{E} \phi^{2} \mathrm{~d} m<\infty$. Then by the same arguments as in [19, Proposition 3.1], we have the following. 


\section{LEMMA 4.2.}

(1) $\mathbf{Z}$ is a $\phi^{2} m$-symmetric right process on $E$.

(2) Let $\left(\mathcal{E}^{Z}, \mathcal{F}^{Z}\right)$ be the Dirichlet form on $L^{2}\left(E ; \phi^{2} m\right)$ associated with $\mathbf{Z}$. Suppose that $\mu_{\langle u\rangle} \in S_{K}^{1}(\mathbf{X}), \mu=\mu_{1}-\mu_{2}$ and $F=F_{1}-F_{2}$ with $\mu_{1}+N\left(F_{1}\right) \mu_{H} \in$ $S_{L K}^{1}(\mathbf{X}) \cap S_{E K}^{1}(\mathbf{X})$ and $\mu_{2}+N\left(F_{2}\right) \mu_{H} \in S_{L K}^{1}(\mathbf{X}) \cap S_{D}^{1}(\mathbf{X})$. Then $\mathcal{F} \subset \mathcal{F}^{Z}$ and for $f \in \mathcal{F}$,

$$
\begin{aligned}
\mathcal{E}^{Z}(f, f)= & \frac{1}{2} \int_{E} \phi(x)^{2} \mu_{\langle f\rangle}^{c}(\mathrm{~d} x) \\
& +\int_{E \times E \backslash \mathrm{diag}}(f(x)-f(y))^{2} \phi(x) \phi(y)(1+F(x, y)) J(\mathrm{~d} x \mathrm{~d} y) .
\end{aligned}
$$

Moreover, $1 \in \mathcal{F}^{Z}$ and $\mathcal{E}^{Z}(1,1)=0$.

(3) $\mathbf{Z}$ is ergodic in the sense that if $\Lambda \in \mathcal{F}_{\infty}^{0}$ is $\left(\theta_{t}\right)$-invariant, i.e., $\theta_{t}^{-1}(\Lambda)=\Lambda$ for any $t>0$, then $\mathbf{P}_{x}^{Z}(\Lambda)=0$ for all $x \in E$ or $\mathbf{P}_{x}^{Z}(\Lambda)=1$ for all $x \in E$. Here $\mathcal{F}_{\infty}^{0}=\sigma\left\{X_{t}: 0 \leq t<\infty\right\}$.

Let $\mathcal{P}(E)$ be the space of all Borel probability measures on $E$ equipped with the weak topology. For $\omega \in \Omega$ with $t<\zeta(\omega)$, define the normalized occupation time distribution $L_{t}(\omega) \in \mathcal{P}(E)$ by

$$
L_{t}(\omega)(A):=\frac{1}{t} \int_{0}^{t} \mathbf{1}_{A}\left(X_{S}(\omega)\right) \mathrm{d} s, \quad A \in \mathcal{B}(E) .
$$

Consider a rate function $I_{\mathcal{Q}}(v)$ on $\mathcal{P}(E)$ defined by

$$
I_{\mathcal{Q}}(v):= \begin{cases}\mathcal{Q}(f, f) & \text { if } \mathrm{d} v=f^{2} \mathrm{~d} m, f \in \mathcal{D}(\mathcal{Q}), \\ +\infty & \text { otherwise }\end{cases}
$$

In the same way as in [33, Proposition 4.3], $I_{\mathcal{Q}}(v)$ is equal to the so called Donsker-Varadhan type's $I$-function defined by

$$
I(v)=-\inf _{\phi \in \mathcal{D}_{++}(\mathcal{A})} \int_{E} \frac{\mathcal{A} \phi}{\phi} \mathrm{d} v, \quad \text { for } v \in \mathcal{P}(E) .
$$

Here $\mathcal{D}_{++}(\mathcal{A}):=\left.\mathcal{D}_{++}\left(\mathcal{A}^{\partial}\right)\right|_{E}$ with

$$
\mathcal{D}_{++}\left(\mathcal{A}^{\partial}\right):=\left\{\bar{\phi}=S_{\alpha}^{\partial} g \mid \alpha>\beta, g \in C\left(E_{\partial}\right), \inf _{x \in E_{\partial}} g(x)>0\right\}
$$

and $\beta$ appeared above is the constant in (3.4). For $\phi \in \mathcal{D}_{++}(\mathcal{A})$ with $\phi=\left.\bar{\phi}\right|_{E}, \bar{\phi} \in \mathcal{D}_{++}\left(\mathcal{A}^{\partial}\right)$, we set $\mathcal{A} \phi:=\alpha \phi-g$ on $E$ and $\mathcal{A}^{\partial} \bar{\phi}:=\alpha \bar{\phi}-g$ on $E_{\partial}$. Clearly, $\mathcal{A}^{\partial} \bar{\phi}=\mathcal{A} \phi$ on $E$ and $\mathcal{A}^{\partial \bar{\phi}}(\partial)=\alpha \bar{\phi}(\partial)-g(\partial)=0$.

Proof of TheOREM 1.1. (1): In view of Lemma 4.2, $\mathbf{Z}$ is ergodic with invariant measure $\phi^{2} \mathrm{~d} m$. So the lower bound (1) of the present theorem can be proved by exactly the same manner as that in [33, Proposition 4.1]. 
(2): To prove the upper bound, we have only to imitate the argument in [33, Proposition 4.2]. Indeed, for $\phi \in \mathcal{D}_{++}(\mathcal{A})$, we can define the supermartingale multiplicative functional $Z_{t}$ as like in (4.4). Though $\phi$ may not belong to $\mathcal{F}$, we can define a local MAF $M^{\bar{\phi}}$ by $M_{t}^{\bar{\phi}}:=\int_{0}^{t} e^{\alpha s} e_{-A}(s) \mathrm{d} M_{s}^{u, \mu, F, \bar{\phi}}-M_{t}^{\bar{\phi}, F}$, where $\left\{M_{t}^{u, \mu, \bar{\phi}, F}\right\}_{t \in[0, \infty[}$ defined like (4.1) by use of $\bar{\phi}$ and $\mathcal{A}^{\partial}$ is a $\mathbf{P}_{x}$-martingale for all $x \in E$. Note that Lemma 3.2(3) and $\bar{\phi}(\partial)=\frac{g(\partial)}{\alpha}>0$ together imply $\inf _{x \in E} \phi(x) \geq \inf _{x \in E_{\partial}} \bar{\phi}(x)>0$ for $\phi \in \mathcal{D}_{++}(\mathcal{A})$. Then the solution $Z_{t}$ of the Doléans-Dade equation (4.2) with

$$
\check{M}_{t}:=\int_{0}^{t} \frac{1}{\bar{\phi}\left(X_{s-}\right)} \mathrm{d}\left(M_{s}^{\bar{\phi}}+M_{s}^{\bar{\phi}, F}\right), \quad t \in[0, \infty[,
$$

has a similar expression as in (4.4) in terms of $\bar{\phi}$ and $\mathcal{A}^{\partial}$. Since $\left\{Z_{t}\right\}_{t \in[0, \infty[}$ is a supermartingale with $Z_{0}=1$,

and thus

$$
\mathbf{E}_{x}\left[e_{A}(t) \frac{\bar{\phi}\left(X_{t}\right)}{\bar{\phi}\left(X_{0}\right)} \exp \left(-\int_{0}^{t} \frac{\mathcal{A}^{\partial} \bar{\phi}\left(X_{S}\right)}{\bar{\phi}\left(X_{S}\right)} \mathrm{d} s\right)\right] \leq 1
$$

$$
\sup _{x \in E} \mathbf{E}_{x}\left[e_{A}(t) \exp \left(-\int_{0}^{t} \frac{\mathcal{A} \phi\left(X_{S}\right)}{\phi\left(X_{S}\right)} \mathrm{d} s\right): t<\zeta\right] \leq \frac{\sup _{x \in E} \phi(x)}{\inf _{x \in E} \phi(x)} .
$$

Hence, for any Borel set $C \subset \mathcal{P}(E)$,

$$
\varlimsup_{t \rightarrow \infty} \frac{1}{t} \log \sup _{x \in E} \mathbf{E}_{x}\left[e_{A}(t): L_{t} \in C, t<\zeta\right] \leq \inf _{\phi \in \mathcal{D}_{++}(\mathcal{A})} \sup _{v \in C} \int_{E} \frac{\mathcal{A} \phi}{\phi} d v .
$$

In particular, the inequality (4.5) yields that for any compact set $K \subset \mathcal{P}(E)$,

$$
\varlimsup_{t \rightarrow \infty} \frac{1}{t} \log \sup _{x \in E} \mathbf{E}_{x}\left[e_{A}(t): L_{t} \in K, t<\zeta\right] \leq-\inf _{v \in K} I(v)
$$

holds by using a finite open covering $\left\{N_{i}=N\left(v_{i}\right)\right\}$ of $K$. Here we use that any $\phi \in$ $\mathcal{D}_{++}(\mathcal{A})$, hence $\mathcal{A} \phi / \phi$, is bounded upper semi continuous on $E$. Now we prove the upper semi continuities of such $\phi$ and $\mathcal{A} \phi / \phi$. Assume first $\mu_{2}+N\left(F_{2}\right) \mu_{H} \in S_{K}^{1}(\mathbf{X})$. Applying Proposition 2.1(3) to the transformed process $\mathbf{Y}$, for a sufficiently small $s>0$ and $\mu_{2}+N\left(F_{2}\right) \mu_{H} \in S_{K}^{1}(\mathbf{X})$, we have the continuity of $x \mapsto \mathbf{E}_{x}^{Y}\left[\operatorname{Exp}\left(A^{\mu_{u}}+A^{\mu, F}\right)_{s}\right]$, because $\log \left[\operatorname{Exp}\left(A^{\mu_{u}}+A^{\mu, F}\right)_{t}\right]=A_{t}^{\mu_{u}+\mu}+A_{t}^{\log (1+F)}$ is a difference of PAF of Kato class in the strict sense under $\mathbf{Y}$ from Lemma 3.5(3). Owing to the strong Feller property of $\left(Q_{t}\right)_{t>0}$ with Remark 3.1, if $\mu_{2}+N\left(F_{2}\right) \mu_{H} \in S_{K}^{1}(\mathbf{X})$, for such a sufficiently small $s>0$, we have the continuity of

$$
\begin{aligned}
x \mapsto & Q_{s}^{\partial} \mathbf{1}_{E_{\partial}}(x)=\mathbf{E}_{x}\left[e_{A}(s)\right]=e^{-u(x)} \mathbf{E}_{x}^{Y}\left[\operatorname{Exp}\left(A^{\mu_{u}}+A^{\mu, F}\right)_{s} e^{u}\left(X_{S}\right)\right] \\
= & e^{-u(x)} \mathbf{E}_{x}^{Y}\left[\operatorname{Exp}\left(A^{\mu_{u}}+A^{\mu, F}\right)_{s}\left(e^{u}-e^{u(\partial)}\right)\left(X_{s}\right)\right] \\
& +e^{u(\partial)-u(x)} \mathbf{E}_{x}^{Y}\left[\operatorname{Exp}\left(A^{\mu_{u}}+A^{\mu, F}\right)_{s}\right] \\
= & Q_{s}\left(\mathbf{1}_{E}\left(1-e^{u(\partial)-u}\right)\right)(x)+e^{u(\partial)-u(x)} \mathbf{E}_{x}^{Y}\left[\operatorname{Exp}\left(A^{\mu_{u}}+A^{\mu, F}\right)_{s}\right] .
\end{aligned}
$$

Applying the strong Feller property of $\left(Q_{t}\right)_{t>0}$ again,

$$
Q_{t+s}^{\partial} \mathbf{1}_{E_{\partial}}=Q_{s}^{\partial}\left(Q_{t}^{\partial} \mathbf{1}_{E_{\partial}}-\mathbf{1}_{E_{\partial}}\right)+Q_{s}^{\partial} \mathbf{1}_{E_{\partial}}
$$




$$
=Q_{s}\left(Q_{t}^{\partial} \mathbf{1}_{E_{\partial}}-\mathbf{1}_{E_{\partial}}\right)+Q_{s}^{\partial} \mathbf{1}_{E_{\partial}}
$$

is also continuous on $E$ for any $t>0$ and such a small $s>0$. This shows the continuity of $x \mapsto Q_{s}^{\partial} \mathbf{1}_{E_{\partial}}(x)$ on $E$ for any $s>0$ under $\mu_{2}+N\left(F_{2}\right) \mu_{H} \in S_{K}^{1}(\mathbf{X})$. We obtain the continuity of $x \mapsto S_{\alpha}^{\partial} \mathbf{1}_{E_{\partial}}(x)$ on $E$ for $\alpha>\beta$ by applying the dominated convergence theorem and (3.3) under $\mu_{2}+N\left(F_{2}\right) \mu_{H} \in S_{K}^{1}(\mathbf{X})$. Next we prove the upper semi continuity of $x \mapsto S_{\alpha}^{\partial} \mathbf{1}_{E_{\partial}}(x)$ on $E$ for $\alpha>\beta$ without assuming $\mu_{2}+N\left(F_{2}\right) \mu_{H} \in S_{K}^{1}(\mathbf{X})$. This can be obtained by approximating the killing part by Kato class PAFs in the strict sense. Therefore any $\phi \in \mathcal{D}_{++}(\mathcal{A})$ and $\mathcal{A} \phi / \phi=\alpha-g / \phi$ are upper continuous on $E$ by $\phi=\left.S_{\alpha}^{\partial} g\right|_{E}=$ $S_{\alpha}\left(g-g(\partial) \mathbf{1}_{E}\right)+\left.g(\partial) S_{\alpha}^{\partial} \mathbf{1}_{E_{\partial}}\right|_{E}$.

Recall that $I(v)=I_{\mathcal{Q}}(v)$ in a similar way of [33, Proposition 4.3]. This implies the desired result.

(3): Recall that (3.4) holds for any $\alpha>\beta$ with some $C>1$ and $\beta>0$. More strongly, we have that there exist $C>1, \beta>0$ and $r>1$ such that

$$
\left\|S_{\alpha}^{\partial} f\right\|_{\infty} \leq\left(\frac{C}{\alpha-\beta}\right)^{\frac{r-1}{r}}\left\|R_{\alpha}^{\partial}|f|^{r}\right\|_{\infty}^{1 / r} \text {, for any } f \in \mathcal{B}_{b}\left(E_{\partial}\right) .
$$

Indeed, taking $q>1$ with $q\left(\mu_{1}+N\left(F_{1}\right) \mu_{H}\right) \in S_{E K}^{1}(\mathbf{X})$, we have

$$
\begin{aligned}
Q_{t}^{\partial}|f|(x) & \leq e^{2\|u\|_{\infty}} \mathbf{E}_{x}\left[Y_{t} e^{A_{t}^{\mu_{u}}} \operatorname{Exp}\left(A^{\mu_{1}, F_{1}}\right)_{t}|f|\left(X_{t}\right)\right] \\
& \leq e^{2\|u\|_{\infty}} \mathbf{E}_{x}\left[e^{p A_{t}^{\mu_{u}}}\right]^{1 / p} \mathbf{E}_{x}\left[Y_{t}^{q} \operatorname{Exp}\left(A^{\mu_{1}, F_{1}}\right)_{t}^{q}\right]^{1 / q} \mathbf{E}_{x}\left[|f|\left(X_{t}\right)^{r}\right]^{1 / r} \\
& \leq e^{2\|u\|_{\infty}}\left(C_{p} e^{\beta_{p} t}\right)^{1 / p}\left(C_{u, q} e^{\beta_{u, q} t}\right)^{1 / q}\left(P_{t}^{\partial}|f|^{r}(x)\right)^{1 / r}
\end{aligned}
$$

for some $C_{p}, C_{u, q}>1$ and $\beta_{p}, \beta_{u, q}>0$ with $\frac{1}{p}+\frac{1}{q}+\frac{1}{r}=1, p, q, r>1$. Then we can obtain (4.6). Hereafter we always take $\alpha>C+\beta>1$, in particular, $\left\|S_{\alpha}^{\partial} \mathbf{1}_{E_{\partial}}\right\|_{\infty}<1$. Take $\varepsilon \in] 0,1 /(3 \alpha)$ [. Since $m \in S_{K_{\infty}^{+}}^{1}(\mathbf{X})$, we can take a compact set $K_{\varepsilon}$ such that $\left\|R_{1} \mathbf{1}_{K_{\varepsilon}^{c}}\right\|_{\infty}<\varepsilon^{r}$, hence $\left\|S_{\alpha} \mathbf{1}_{K_{\varepsilon}^{c}}\right\|_{\infty}<\varepsilon$ for $\alpha>C+\beta(>1)$. Here $K_{\varepsilon}^{c}:=E \backslash K_{\varepsilon}$. We prepare larger domain for generator:

$$
\mathcal{D}_{++}^{*}\left(\mathcal{A}^{\partial}\right):=\left\{\bar{\phi}=S_{\alpha}^{\partial} g \mid \alpha>\beta, g \in \mathcal{B}_{b}\left(E_{\partial}\right), \inf _{x \in E_{\partial}} g(x)>0\right\}
$$

and $\mathcal{A}^{\partial} \bar{\phi}:=\alpha \bar{\phi}-g$ for $\bar{\phi}=S_{\alpha}^{\partial} g \in \mathcal{D}_{++}^{*}\left(\mathcal{A}^{\partial}\right)$, where $\beta$ is the constant appeared in (3.4). Then for $\bar{\phi} \in \mathcal{D}_{++}^{*}\left(\mathcal{A}^{\partial}\right), Z_{t}$ can be defined to be a supermartingale multiplicative functional having a similar expression with (4.4) by way of the above $\bar{\phi}$ as in the proof of (2). Set $V_{\varepsilon}:=-\frac{\mathcal{A}^{\partial} \phi_{\varepsilon}}{\phi_{\varepsilon}}$ with $\phi_{\varepsilon}:=S_{\alpha}^{\partial}\left(\mathbf{1}_{K_{\varepsilon}^{c}}+\varepsilon \mathbf{1}_{E_{\partial}}\right) \in \mathcal{D}_{++}^{*}(\mathcal{A})$. Then

$$
V_{\varepsilon}=\frac{\mathbf{1}_{K_{\varepsilon}^{c}}+\varepsilon \mathbf{1}_{E_{\partial}}}{S_{\alpha}^{\partial}\left(\mathbf{1}_{K_{\varepsilon}^{c}}+\varepsilon \mathbf{1}_{E_{\partial}}\right)}-\alpha .
$$

The rest of the description of the proof is quite similar as in the proof of [12, Theorem 1.1(3)]. So we omit it. 
Proof of Corollary 1.1. The proof of (1) is similar to the proof of Proposition 3.1 in [40]. The proof of (2) is an easy consequence of Theorem 1.1 (cf. Theorem 4.1 in [40]). The proof of (3) is similar to the proof of Corollary 4.1 in [40].

Let us denote by $\left\|Q_{t}\right\|_{p, p}$ the operator norm of $Q_{t}$ from $L^{p}(E ; m)$ to $L^{p}(E ; m)$ and put

$$
\lambda_{p}:=\lambda_{p}(u, \mu, F):=-\lim _{t \rightarrow \infty} \frac{1}{t} \log \left\|Q_{t}\right\|_{p, p}, \quad 1 \leq p \leq \infty
$$

as in Section 1.

The proof of Theorem 1.2 is the same as that of Theorems 1.2 in [12]. So we omit it.

REMARK 4.1.

(1) In Takeda [37] (resp. Takeda-Tawara [40]), Theorem 1.1 is proved under the conditions $u=F=0$ and $|\mu| \in S_{K_{\infty}^{+}}^{1}(\mathbf{X})\left(\right.$ resp. $u=0,|\mu| \in S_{K_{\infty}^{+}}^{1}(\mathbf{X})$ and $|F| \in A_{2}(\mathbf{X})$ ), where $A_{2}(\mathbf{X})$ denotes a class of jump functions satisfying a positive order Greentightness with respect to conditional processes (see [40] for the definitions). The method of the proof of Theorem 1.1 in [37, 40] is based on the gaugeability of Feynman-Kac functional. Our proof of Theorem 1.1 does not use the conditions for the gaugeability.

(2) If we assume $\mu_{\langle u\rangle} \in S_{K}^{1}(\mathbf{X}), \mu=\mu_{1}-\mu_{2} \in S_{K}^{1}(\mathbf{X})-S_{K}^{1}(\mathbf{X})$ and $F=F_{1}-F_{2} \in$ $J_{K}^{1}(\mathbf{X})-J_{K}^{1}(\mathbf{X})$ then we can obtain the same conclusions as in Theorems 1.1, 1.2(1) and Corollary 1.1 without assuming the Feller property of $\mathbf{X}$. Indeed, under these conditions, $\left(Q_{t}\right)_{t>0}$ has the strong Feller property without the Feller property of $\mathbf{X}$ by Remark 3.2. For the proof of the assertion of Theorem 1.1(1), we only need the absolute continuity condition of $\left(Q_{t}\right)_{t>0}$ with respect to $\phi^{2} m$, which is guaranteed by the strong Feller property of $\left(Q_{t}\right)_{t>0}$. In the proof of Theorem 1.1(2), for $\alpha>\beta$, we have the continuity of $S_{\alpha}^{\partial} \mathbf{1}_{E_{\partial}}$ on $E$, consequently, for $\phi \in \mathcal{D}_{++}(\mathcal{A})$, we have $\phi=\left.S_{\alpha}^{\partial} g\right|_{E} \in C_{b}(E)$ with $g \in C\left(E_{\partial}\right)$ and $\mathcal{A} \phi=\alpha \phi-g \in C_{b}(E)$. Hence $\mathcal{A} \phi / \phi \in C_{b}(E)$, which yields the upper estimates. The proof of Theorem 1.1(3) under the conditions $\mu_{\langle u\rangle} \in S_{K}^{1}(\mathbf{X}), \mu=\mu_{1}-\mu_{2} \in S_{K}^{1}(\mathbf{X})-S_{K}^{1}(\mathbf{X})$ and $F=$ $F_{1}-F_{2} \in J_{K}^{1}(\mathbf{X})-J_{K}^{1}(\mathbf{X})$ does not use the Feller property of $\mathbf{X}$.

(3) In Theorem 1.2(2),(3), we used the Feller property of $\mathbf{X}$ and the regularity of the open set $G$ for the doubly Feller property of the part process $\mathbf{X}_{G}$ on $G$. Instead of the Feller property of $\mathbf{X}$ and the regularity of $G$, the strong Feller property of $\mathbf{X}_{G}$ yields the same conclusion provided we replace the hypotheses for Theorem 1.2 with $\mu=\mu_{1}-\mu_{2} \in S_{K}^{1}(\mathbf{X})-S_{K}^{1}(\mathbf{X})$ and $F=F_{1}-F_{2} \in J_{K}^{1}(\mathbf{X})-J_{K}^{1}(\mathbf{X})$ in Theorem 1.2(2).

Proof of Theorem 1.3. For $\mu=\mu_{1}-\mu_{2} \in S_{K_{\infty}^{+}}^{1}(\mathbf{X})-S_{L K}^{1}(\mathbf{X}) \cap S_{D}^{1}(\mathbf{X}), F=$ $F_{1}-F_{2} \in J_{K_{\infty}^{+}}^{1}(\mathbf{X})-J_{L K}^{1}(\mathbf{X}) \cap J_{D}^{1}(\mathbf{X})$ and $\mu_{\langle u\rangle} \in S_{K_{\infty}^{+}}^{1}(\mathbf{X})$, define the modified $I$-function $I^{\partial}$ on $\mathcal{P}\left(E_{\partial}\right)$ by

$$
I^{\partial}(\nu)=-\inf _{\bar{\phi} \in \mathcal{D}_{++}\left(\mathcal{A}^{\partial}\right)} \int_{E_{\partial}} \frac{\mathcal{A}^{\partial} \bar{\phi}}{\bar{\phi}} \mathrm{d} \nu,
$$


where $\mathcal{A}^{\partial} \bar{\phi}=\alpha \bar{\phi}-g$ for $\bar{\phi}=S_{\alpha}^{\partial} g \in \mathcal{D}_{++}\left(\mathcal{A}^{\partial}\right)$. By Theorem 3.1, any $\bar{\phi}:=S_{\alpha}^{\partial} g, g \in C_{+}\left(E_{\partial}\right)$ is upper semi continuous on $E_{\partial}$. Hence, $\mathcal{A}^{\partial} \bar{\phi} / \bar{\phi}=\alpha-g / \bar{\phi}$ is upper semi continuous on $E_{\partial}$. Note that $\mathcal{P}\left(E_{\partial}\right)$ is compact with respect to the weak topology. Therefore, we can derive the following inequality in the similar way as one in the proof of the upper bound of Theorem $1.1(2)$.

$$
\varlimsup_{t \rightarrow \infty} \frac{1}{t} \log \sup _{x \in E} \mathbf{E}_{x}\left[e_{A}(t): t<\zeta\right] \leq-\inf _{v \in \mathcal{P}\left(E_{\partial}\right)} I^{\partial}(v) .
$$

Since $\mathcal{P}\left(E_{\partial}\right) \backslash\left\{\delta_{\partial}\right\}$ and $\left.] 0,1\right] \times \mathcal{P}(E)$ are in one to one correspondence by the map

$$
\left.\left.v \in \mathcal{P}\left(E_{\partial}\right) \backslash\left\{\delta_{\partial}\right\} \mapsto(v(E), \widehat{v}) \in\right] 0,1\right] \times \mathcal{P}(E) \text { for } \widehat{v}(\cdot):=\frac{v(\cdot)}{v(E)},
$$

and $I^{\partial}(v)=I(v)=-\inf _{\phi \in \mathcal{D}_{++}(\mathcal{A})} v(E) \int_{E} \frac{\mathcal{A} \phi}{\phi} \mathrm{d} \widehat{v}=v(E) I_{\mathcal{Q}}(\widehat{v})$, we have

$$
\begin{aligned}
\varlimsup_{t \rightarrow \infty} \frac{1}{t} \log \sup _{x \in E} \mathbf{E}_{x}\left[e_{A}(t): t<\zeta\right] & \leq-\inf _{\theta \in[0,1]}\left(\theta \inf _{\nu \in \mathcal{P}(E)} I_{\mathcal{Q}}(v)\right) \\
& =-\inf _{\theta \in[0,1]}\left(\theta \lambda_{2}\right) .
\end{aligned}
$$

The left-hand side of (4.7) equals $-\lambda_{\infty}$ because $\sup _{x \in E} \mathbf{E}_{x}\left[e_{A}(t): t<\zeta\right]=\left\|Q_{t}\right\|_{\infty, \infty}$. Hence we have

$$
\lambda_{\infty} \geq \inf _{\theta \in[0,1]}\left(\theta \lambda_{2}\right) .
$$

If $\lambda_{2} \leq 0$, then the right-hand side of (4.8) is equal to $\lambda_{2}$. So we have $\lambda_{\infty} \geq \lambda_{2}$. The converse inequality always holds because

$$
\left\|Q_{t}\right\|_{2,2} \leq\left\|Q_{t}\right\|_{p, p} \leq\left\|Q_{t}\right\|_{\infty, \infty}, \quad 1 \leq p \leq \infty
$$

by the symmetry and the positivity of $Q_{t}$. Now, we see that the $L^{p}$-independence holds if $\lambda_{2} \leq 0$. The latter assertion of the present theorem can be proved similarly to Theorem 1.3 in [12] by using Lemmas 3.3 and 3.5. So we omit the proof.

Proof of Corollary 1.2. Suppose that $\mu_{\langle u\rangle} \in S_{K_{\infty}^{+}}^{1}(\mathbf{X}), \mu_{1} \in S_{K_{\infty}^{+}}^{1}(\mathbf{X}), \mu_{2}=0$, $F_{1} \in J_{K_{\infty}^{+}}^{1}(\mathbf{X}), F_{2}=0$ hold. In view of Beurling-Deny formula (see [16]), for $f \in \mathcal{F} \cap C_{0}(E)$, we have

$$
\begin{aligned}
\mathcal{Q}(f, f)= & \mathcal{E}(f, f)+\int_{E} f \mathrm{~d} \mu_{\langle f, u\rangle}^{c}+2 \iint_{E \times E} f(x)(f(x)-f(y))(u(x)-u(y)) J(\mathrm{~d} x \mathrm{~d} y) \\
& +\int_{E} f^{2} u \mathrm{~d} \kappa-\int_{E} f^{2} \mathrm{~d} \mu_{1}-\iint_{E \times E} f(x) f(y) F_{1}(x, y) N(x, \mathrm{~d} y) \mu_{H}(\mathrm{~d} x) \\
\leq & \mathcal{E}(f, f)+\sqrt{2} \mathcal{E}(f, f)^{1 / 2}\left(\int_{E} f^{2} \mathrm{~d} \mu_{\langle u\rangle}\right)^{1 / 2} \\
& -\iint_{E \times E} f(x)(f(y)-f(x)) F_{1}(x, y) N(x, \mathrm{~d} y) \mu_{H}(\mathrm{~d} x) \\
\leq & \mathcal{E}(f, f)+\sqrt{2} \mathcal{E}(f, f)^{1 / 2}\left(\left\|R_{1} \mu_{\langle u\rangle}\right\|_{\infty} \mathcal{E}_{1}(f, f)\right)^{1 / 2}
\end{aligned}
$$




$$
\begin{aligned}
& +\int_{E}|f(x)|\left(\int_{E}(f(y)-f(x))^{2} N(x, \mathrm{~d} y)\right)^{1 / 2}\left(N\left(\left(F_{1}\right)^{2}\right)(x)\right)^{1 / 2} \mu_{H}(\mathrm{~d} x) \\
\leq & \mathcal{E}(f, f)+\sqrt{2} \mathcal{E}(f, f)^{1 / 2}\left(\left\|R_{1} \mu\langle u\rangle\right\|_{\infty} \mathcal{E}_{1}(f, f)\right)^{1 / 2} \\
& +\left(\int_{E} f(x)^{2} N\left(\left(F_{1}\right)^{2}\right)(x) \mu_{H}(\mathrm{~d} x)\right)^{1 / 2}\left(\int_{E}(f(y)-f(x))^{2} N(x, \mathrm{~d} y) \mu_{H}(\mathrm{~d} x)\right)^{1 / 2} \\
\leq & \mathcal{E}(f, f)+\sqrt{2} \mathcal{E}(f, f)^{1 / 2}\left(\left\|R_{1} \mu_{\langle u\rangle}\right\|_{\infty} \mathcal{E}_{1}(f, f)\right)^{1 / 2} \\
& +\left(\left\|R_{1} N\left(\left(F_{1}\right)^{2}\right) \mu_{H}\right\|_{\infty} \mathcal{E}_{1}(f, f)\right)^{1 / 2} \mathcal{E}(f, f)^{1 / 2} .
\end{aligned}
$$

Since $\lambda_{2}(0,0,0) \leq 0$, we have $\lambda_{2}(0,0,0)=0$. So by taking a sequence $\left\{f_{n}\right\} \subset \mathcal{F} \cap C_{0}(E)$ with $\left\|f_{n}\right\|_{2}=1, \mathcal{E}\left(f_{n}, f_{n}\right) \rightarrow 0$ as $n \rightarrow \infty$, we have $\mathcal{Q}\left(f_{n}, f_{n}\right) \rightarrow 0$ as $n \rightarrow \infty$, which implies $\lambda_{2}(u, \mu, F) \leq 0$ under the conditions. If we further assume the transience of $\mathbf{X}$, then for $v \in S_{K_{\infty}}^{1}(\mathbf{X})$, we have the Stollmann-Voigt inequality (see [31])

$$
\int_{E} f^{2} \mathrm{~d} v \leq\|R v\|_{\infty} \mathcal{E}(f, f) \text { for any } f \in \mathcal{F}_{e} .
$$

The proof of the second statement is easily seen from this and a similar argument by noting $N\left(\left(F_{2}\right)^{2}\right) \mu_{H} \in S_{K_{\infty}}^{1}(\mathbf{X})$.

\section{Examples.}

EXAMPLE 5.1 (Birth and Death Process, cf. [13]). Let $\mathbf{X}$ be a birth and death process, that is, a time homogeneous Markov process with transition function $P_{i j}(t)$ such that

$$
P_{i j}(t) \geq 0, \quad \sum_{k=0}^{\infty} P_{i k}(t) \leq 1, \quad P_{i j}(t+s)=\sum_{k=0}^{\infty} P_{i k}(t) P_{k j}(s) .
$$

Moreover,

$$
\begin{cases}P_{i+1}(t)=\lambda_{i} t+o(t) & \text { as } t \rightarrow 0, \quad i \geq 0, \\
P_{i i}(t)=1-\left(\lambda_{i}+\mu_{i}\right) t+o(t) & \text { as } t \rightarrow 0, \quad i \geq 0, \\
P_{i-1}(t)=\mu_{i} t+o(t) & \text { as } t \rightarrow 0, i \geq 1, \\
P_{i j}(0)=\delta_{i j}:=\left\{\begin{array}{ll}
1 & i=j \\
0 & i \neq j
\end{array},\right. & \end{cases}
$$

where $\lambda_{i}(i=0,1,2, \ldots)$ and $\mu_{i}(i=1,2,3, \ldots)$ are positive constants and $\mu_{0}:=0$. We set

$$
x_{0}=0, \quad x_{1}=\frac{1}{\lambda_{0}}, \quad x_{n}=\frac{1}{\lambda_{0}}+\sum_{i=1}^{n-1} \frac{1}{\lambda_{i} m_{i}}
$$

with

$$
m_{0}=1, \quad m_{i}=\frac{\lambda_{0} \lambda_{1} \cdots \lambda_{i-1}}{\mu_{1} \mu_{2} \cdots \mu_{i}}, \quad(i=1,2, \ldots) .
$$

We further set $E:=\left\{x_{i}\right\}_{i=0}^{\infty}$ and a measure $m$ by $m\left(\left\{x_{i}\right\}\right):=m_{i}$. Then $m$ is a Radon measure on $E$ and $\mathbf{X}$ is $m$-symmetric, $P_{i j}(t) m_{i}=P_{j i}(t) m_{j}$ for any $i, j \in \mathbb{N} \cup\{0\}$. We prepare the following conditions: For $x_{\infty}:=\lim _{i \rightarrow \infty} x_{i}$, 
(A1) $x_{\infty}$ is a regular boundary, that is, $x_{\infty}<\infty$ and $\sum_{i=0}^{\infty} m_{i}<\infty$.

(A2) $x_{\infty}$ is a natural boundary, that is, $x_{\infty}=\infty$ and $\sum_{i=0}^{\infty} m_{i}=\infty$.

Suppose that (A1) holds. Then the one point compactification $E_{\partial}$ of $E$ can be regarded as $E \cup\left\{x_{\infty}\right\}$. Let us introduce a symmetric bilinear form $(\mathcal{E}, \mathcal{F})$ on $L^{2}(E ; m)$ by

$$
\left\{\begin{array}{l}
\mathcal{F}:=\left\{\left.f \in C_{\infty}(E)\left|\sum_{i=0}^{\infty}\right| D f\left(x_{i}\right)\right|^{2}\left(x_{i+1}-x_{i}\right)<\infty\right\}, \\
\mathcal{E}(f, g):=\sum_{i=0}^{\infty} D f\left(x_{i}\right) D g\left(x_{i}\right)\left(x_{i+1}-x_{i}\right) \quad f, g \in \mathcal{F},
\end{array}\right.
$$

where $D f\left(x_{i}\right):=\frac{f\left(x_{i+1}\right)-f\left(x_{i}\right)}{x_{i+1}-x_{i}}$ and $C_{\infty}(E):=\left\{f \in C(E) \mid \lim _{i \rightarrow \infty} f\left(x_{i}\right)=0\right\}$. Then $(\mathcal{E}, \mathcal{F})$ is an irreducible transient regular Dirichlet form on $L^{2}(E ; m)$ in the same way of the proof of Lemma 5.1 in [24] (cf. [21]). The corresponding process $\mathbf{X}$ of $(\mathcal{E}, \mathcal{F})$ is an $m$-symmetric one dimensional generalized diffusion process on $E$ with a regular boundary $x_{\infty}<\infty$ (cf. [28]). Since $1 \in L^{2}(E ; m)$, we see $R_{\alpha} 1 \in \mathcal{F} \subset C_{\infty}(E)$, hence $m \in S_{K_{\infty}^{+}}^{1}(\mathbf{X})$. Moreover, by way of Ascoli-Arzela's Theorem, we see $\mathcal{F}_{e}=\mathcal{F}$. The form (5.1) can be rewritten to

$$
\left\{\begin{array}{l}
\mathcal{F}:=\left\{f \in C_{\infty}(E) \mid \int_{E \times E}(f(x)-f(y))^{2} J(\mathrm{~d} x \mathrm{~d} y)<\infty\right\} \\
\mathcal{E}(f, g):=\int_{E \times E}(f(x)-f(y))(g(x)-g(y) J(\mathrm{~d} x \mathrm{~d} y) \quad f, g \in \mathcal{F}
\end{array}\right.
$$

with $J(\mathrm{~d} x \mathrm{~d} y)=\sum_{i=0}^{\infty} \frac{1}{x_{i+1}-x_{i}} \delta_{x_{i}}(\mathrm{~d} x) \delta_{x_{i+1}}(\mathrm{~d} y)$, where $\delta_{x}$ stands for the Dirac measure at $x$.

Next suppose that $(\mathbf{A 2})$ holds. Note that any function on $E$ is always continuous. We consider $(\mathcal{E}, \mathcal{F})$ on $L^{2}(E ; m)$ by

$$
\left\{\begin{array}{l}
\mathcal{F}:=\left\{\left.f \in L^{2}(E ; m)\left|\sum_{i=0}^{\infty}\right| D f\left(x_{i}\right)\right|^{2}\left(x_{i+1}-x_{i}\right)<\infty\right\}, \\
\mathcal{E}(f, g):=\sum_{i=0}^{\infty} D f\left(x_{i}\right) D g\left(x_{i}\right)\left(x_{i+1}-x_{i}\right) \quad f, g \in \mathcal{F}
\end{array}\right.
$$

Then $(\mathcal{E}, \mathcal{F})$ is an irreducible recurrent regular Dirichlet form on $L^{2}(E ; m)$. We then have $\mathcal{F}_{e}:=\left\{\left.f \in C(E)\left|\sum_{i=0}^{\infty}\right| D f\left(x_{i}\right)\right|^{2}\left(x_{i+1}-x_{i}\right)<\infty\right\}$ and $\mathcal{E}(f, g)$ for $f, g \in \mathcal{F}_{e}$ has the same expression as well as for $f, g \in \mathcal{F}$. The form (5.3) also can be rewritten to a similar form to (5.2). The corresponding process $\mathbf{X}$ is an $m$-symmetric one dimensional generalized diffusion process on $E$ with a natural boundary $x_{\infty}=\infty$.

In either (A1) or (A2), the Lévy system $(N, H)$ of $\mathbf{X}$ is determined by

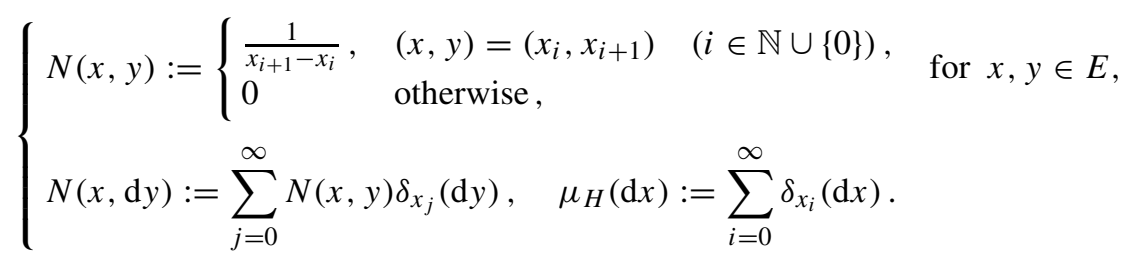

Now we fix a function $u \in \dot{\mathcal{F}}_{\text {loc }} \cap C\left(E_{\partial}\right)$. We further consider 
(A3) $\left\{\lambda_{i} \mid i \in \mathbb{N} \cup\{0\}\right\}$ is bounded. (A4) $u \in C_{\infty}(E)$.

From

$$
\mu_{\langle u\rangle}(\mathrm{d} x)=\sum_{i=0}^{\infty} \frac{\left(u\left(x_{i+1}\right)-u\left(x_{i}\right)\right)^{2}}{x_{i+1}-x_{i}} \delta_{x_{i}}(\mathrm{~d} x),
$$

we have $\mu_{\langle u\rangle} \ll m$ and its density $\Gamma(u):=\mathrm{d} \mu_{\langle u\rangle} / \mathrm{d} m$ is given by

$$
\Gamma(u)\left(x_{i}\right)=\frac{\left(u\left(x_{i+1}\right)-u\left(x_{i}\right)\right)^{2}}{x_{i+1}-x_{i}} \frac{1}{m_{i}}=\left(u\left(x_{i+1}\right)-u\left(x_{i}\right)\right)^{2} \lambda_{i} .
$$

Then $\Gamma(u) \in L^{\infty}(E ; m)$ under (A3). This implies $\mu_{\langle u\rangle} \in S_{K_{\infty}^{+}}^{1}(\mathbf{X})$ under (A1) and (A3), because of $m \in S_{K_{\infty}^{+}}^{1}(\mathbf{X})$ under (A1). On the other hand, under (A3) and (A4), we have $\Gamma(u) \in C_{\infty}(E)$, hence $R_{1} \mu_{\langle u\rangle}=R_{1} \Gamma(u) \in C_{\infty}(E)$ by the doubly Feller property of $\mathbf{X}$. Hence $\mu_{\langle u\rangle} \in S_{K_{\infty}^{+}}^{1}(\mathbf{X})$ under (A2), (A3) and (A4).

Therefore, Theorem 1.1 tells us that, under (A1) and (A3) we have the large deviation principle for the Feynman-Kac functional of CAF $N_{t}^{u}$ of 0 -energy and

$$
\lim _{t \rightarrow \infty} \frac{1}{t} \log \sup _{x \in E} \mathbf{E}_{x}\left[e^{N_{t}^{u}}: t<\sigma_{\left\{x_{\infty}\right\}}\right]=-\inf \left\{\mathcal{Q}(f, f) \mid f \in \mathcal{F},\|f\|_{2}=1\right\},
$$

where $(\mathcal{Q}, \mathcal{F})$ is the quadratic form on $L^{2}(E ; m)$ defined by $\mathcal{Q}(f, g):=\mathcal{E}(f, g)+\mathcal{E}(u, f g)$, $f, g \in \mathcal{F}$. As a consequence, we have the $L^{p}$-independence of the spectral radius $\lambda_{p}(u, 0,0)$ of this Feynman-Kac functional by Theorem 1.2(1).

On the other hand, Corollary 1.2 tells us that $\lambda_{p}(u, 0,0)$ is independent of $p$ under (A2), (A3) and (A4) because $\lambda_{2}(0,0,0)=\inf \left\{\mathcal{E}(f, f) \mid f \in \mathcal{F},\|f\|_{2}=1\right\}=0$.

EXAmPle 5.2 (Symmetric Relativistic $\alpha$-stable Process). Take $\alpha \in] 0,2[$ and $m \geq 0$. Let $\mathbf{X}^{\mathrm{R}, \alpha}=\left(\Omega, X_{t}, \mathbf{P}_{x}\right)_{x \in \mathbb{R}^{d}}$ be a Lévy process on $\mathbb{R}^{d}$ with

$$
\mathbf{E}_{0}\left[e^{\sqrt{-1}\left\langle\xi, X_{t}\right\rangle}\right]=\exp \left(-t\left\{\left(|\xi|^{2}+m^{2 / \alpha}\right)^{\alpha / 2}-m\right\}\right) .
$$

If $m>0$, it is called the relativistic $\alpha$-stable process with mass $m$ (see [30]). In particular, if $\alpha=1$ and $m>0$, it is called the relativistic free Hamiltonian process (see [18]). When $m=0, \mathbf{X}^{\mathrm{R}, \alpha}$ is nothing but the usual symmetric $\alpha$-stable process. We consider the case $m>0$. It is known that $\mathbf{X}^{\mathrm{R}, \alpha}$ is transient if and only if $d>2$ under $m>0$. Let $\left(\mathcal{E}^{\mathrm{R}, \alpha}, \mathcal{F}^{\mathrm{R}, \alpha}\right)$ be the Dirichlet form on $L^{2}\left(\mathbb{R}^{d}\right)$ associated with $\mathbf{X}^{\mathrm{R}, \alpha}$. Using Fourier transform $\hat{f}(x):=$ $\frac{1}{(2 \pi)^{d / 2}} \int_{\mathbb{R}^{d}} e^{i\langle x, y\rangle} f(y) \mathrm{d} y$, it follows from Example 1.4.1 of [16] that

$$
\left\{\begin{array}{l}
\mathcal{F}^{\mathrm{R}, \alpha}:=\left\{\left.f \in L^{2}\left(\mathbb{R}^{d}\right)\left|\int_{\mathbb{R}^{d}}\right| \hat{f}(\xi)\right|^{2}\left(\left(|\xi|^{2}+m^{2 / \alpha}\right)^{\alpha / 2}-m\right) \mathrm{d} \xi<\infty\right\} \\
\mathcal{E}^{\mathrm{R}, \alpha}(f, g):=\int_{\mathbb{R}^{d}} \hat{f}(\xi) \overline{\hat{g}}(\xi)\left(\left(|\xi|^{2}+m^{2 / \alpha}\right)^{\alpha / 2}-m\right) \mathrm{d} \xi \quad \text { for } f, g \in \mathcal{F}^{\mathrm{R}, \alpha}
\end{array}\right.
$$

It is shown by Ryznar [30] that the semigroup kernel $p_{t}(x, y)$ of $\mathbf{X}^{\mathrm{R}, \alpha}$ is given by

$$
p_{t}(x, y)=e^{m t} \int_{0}^{\infty}\left(\frac{1}{4 \pi s}\right)^{d / 2} e^{-\frac{|x-y|^{2}}{4 s}} e^{-s m^{2 / \alpha}} \theta_{\alpha / 2}(t, s) \mathrm{d} s,
$$


where $\theta_{\delta}(t, s)$ is the nonnegative function called the subordinator whose Laplace transform is given by

$$
\int_{0}^{\infty} e^{-\lambda s} \theta_{\delta}(t, s) \mathrm{d} s=e^{-t \lambda^{\delta}}
$$

Then we see the conservativeness of $\mathbf{X}^{\mathrm{R}, \alpha}$. It is shown in [9] that the corresponding jumping measure satisfies

$$
J(\mathrm{~d} x \mathrm{~d} y)=J(x, y) \mathrm{d} x \mathrm{~d} y \quad \text { with } J(x, y)=\frac{A(d,-\alpha)}{2} \frac{\Psi\left(m^{1 / \alpha}|x-y|\right)}{|x-y|^{d+\alpha}},
$$

where $A(d,-\alpha)=\frac{\alpha 2^{d+\alpha} \Gamma\left(\frac{d+\alpha}{2}\right)}{2^{d+1} \pi^{d / 2} \Gamma\left(1-\frac{\alpha}{2}\right)}$, and $\Psi(r):=I(r) / I(0)$ with $I(r):=\int_{0}^{\infty} s^{\frac{d+\alpha}{2}} e^{-\frac{s}{4}-\frac{r^{2}}{s}} \mathrm{~d} s$ is a function satisfying $\Psi(r) \asymp e^{-r}\left(1+r^{(d+\alpha-1) / 2}\right)$ near $r=\infty$, and $\Psi(r)=1+\Psi^{\prime \prime}(0) r^{2} / 2+$ $o\left(r^{4}\right)$ near $r=0$. In particular,

$$
\left\{\begin{array}{l}
\mathcal{F}^{\mathrm{R}, \alpha}=\left\{f \in L^{2}\left(\mathbb{R}^{d}\right)\left|\int_{\mathbb{R}^{d} \times \mathbb{R}^{d}}\right| f(x)-\left.f(y)\right|^{2} J(x, y) \mathrm{d} x \mathrm{~d} y<\infty\right\}, \\
\mathcal{E}^{\mathrm{R}, \alpha}(f, g)=\int_{\mathbb{R}^{d} \times \mathbb{R}^{d}}(f(x)-f(y))(g(x)-g(y)) J(x, y) \mathrm{d} x \mathrm{~d} y \quad \text { for } f, g \in \mathcal{F}^{\mathrm{R}, \alpha} .
\end{array}\right.
$$

As noted in Example 5.1 in [23], we have that for each $t_{0}>0$, there exist $C_{i}=$ $C_{i}(\alpha, d)>0, i=1,2$ independent of $t_{0}$ such that for any $\left.t \in\right] 0, t_{0}\left[, x, y \in \mathbb{R}^{d}\right.$

$$
\frac{C_{1}}{t^{d / \alpha}} \Psi_{1}\left(\frac{|x-y|}{t^{1 / \alpha}}\right) \leq p_{t}(x, y) \leq \frac{C_{2}}{t^{d / \alpha}} \frac{e^{m t_{0}}}{\left(1+\frac{|x-y|}{t^{1 / \alpha}}\right)^{d+\alpha}},
$$

where $\Psi_{1}(s):=\int_{1 \vee s^{2}}^{\infty} e^{-\left(m t_{0}\right)^{2 / \alpha} u} \frac{d u}{u^{\frac{d+\alpha}{2}+1}}$. In particular, $\sup _{x, y \in \mathbb{R}^{d}} p_{t}(x, y) \leq C_{2} e^{m t_{0}} / t^{d / \alpha}$ for $t \in] 0, t_{0}[$.

For $m=0$, we have the following estimate which can be obtained from Theorem 2.1 in Blumenthal-Getoor [2]: there exists $C_{i}=C_{i}(\alpha, d)>0, i=1,2$ such that for all $(t, x, y) \in$ ] $0, \infty\left[\times \mathbb{R}^{d} \times \mathbb{R}^{d}\right.$

$$
\frac{C_{1}}{t^{d / \alpha}} \frac{1}{\left(1+\frac{|x-y|}{t^{1 / \alpha}}\right)^{d+\alpha}} \leq p_{t}(x, y) \leq \frac{C_{2}}{t^{d / \alpha}} \frac{1}{\left(1+\frac{|x-y|}{t^{1 / \alpha}}\right)^{d+\alpha}} .
$$

For a signed Borel measure $\mu$ on $\mathbb{R}^{d}, \mu$ is said to be of Kato class with respect to $\mathbf{X}^{\mathrm{R}, \alpha}$ if and only if

$$
\begin{gathered}
\lim _{r \rightarrow 0} \sup _{x \in \mathbb{R}^{d}} \int_{|x-y|<r} \frac{|\mu|(\mathrm{d} y)}{|x-y|^{d-\alpha}}=0 \quad \text { for } d>\alpha \\
\lim _{r \rightarrow 0} \sup _{x \in \mathbb{R}^{d}} \int_{|x-y|<r}\left(\log |x-y|^{-1}\right)|\mu|(\mathrm{d} y)=0 \text { for } d=\alpha(=1), \\
\sup _{x \in \mathbb{R}^{d}} \int_{|x-y| \leq 1}|\mu|(\mathrm{d} y)<\infty \text { for } \alpha>d(=1) .
\end{gathered}
$$


Denote by $\mathbf{K}_{d, \alpha}$ the family of non-negative measures of Kato class with respect to $\mathbf{X}^{\mathrm{R}, \alpha}$. Then we have $\mathbf{K}_{d, \alpha}=S_{K}^{1}\left(\mathbf{X}^{\mathrm{R}, \alpha}\right)$ by [23]. Consequently, the surface measure $\sigma_{r}$ on the $r$-sphere $\partial B_{r}(0)$ is in $\mathbf{K}_{d, \alpha}=S_{K}^{1}\left(\mathbf{X}^{\mathrm{R}, \alpha}\right)$ if and only if $\alpha>1$. This was shown by Port [29] for the case $d>\alpha$ and $m=0$.

From Lemma 3 in [30], for $m>0$, there exists $C(d, m, \alpha)>0$ depending only on $m, d$ and $\alpha$ such that

$$
\sup _{x, y \in \mathbb{R}^{d}} p_{t}(x, y) \leq C(d, m, \alpha) t^{-d / 2} \quad \text { for any } t \geq 1
$$

As we see, for $m=0$ there exists $C(d, \alpha)>0$ depending only on $m, d$ and $\alpha$ such that

$$
\sup _{x, y \in \mathbb{R}^{d}} p_{t}(x, y) \leq C(d, \alpha) t^{-d / \alpha} \quad \text { for any } t>0
$$

We can apply [12, Lemma 5.1(2)] to $\mathbf{X}^{\mathrm{R}, \alpha}$ for $\Phi_{1}:=C_{1} \Psi_{1}, \Phi_{2}(s):=\frac{C_{2} e^{m}}{(1+s)^{d+\alpha}}$ and $\Phi_{2}^{*}(s):=$ $C(d, m, \alpha)$ with $t_{0}=1, d^{*}=d$ and $\beta^{*}=2>\beta=\alpha$ provided $m>0$, and for $\Phi_{1}=C_{1} \Psi_{2}$, $\Phi_{2}=\Phi_{2}^{*}=C_{2} \Psi_{2}$ with $\Psi_{2}(s):=1 /(1+s)^{d+\alpha}$, and $d=d^{*}, \beta^{*}=\beta=\alpha$ provided $m=0$. Hence every $\mu \in \mathbf{K}_{d, \alpha}=S_{K}^{1}\left(\mathbf{X}^{\mathrm{R}, \alpha}\right)$ with $\mu\left(\mathbb{R}^{d}\right)<\infty$ belongs to $S_{K_{\infty}^{+}}^{1}\left(\right.$ to $S_{K_{\infty}}^{1}\left(\mathbf{X}^{\mathrm{R}, \alpha}\right)$ provided $d>2)$. Hence $\sigma_{r} \in S_{K_{\infty}^{+}}^{1}\left(\mathbf{X}^{\mathrm{R}, \alpha}\right)\left(\right.$ resp. $\left.\left.\sigma_{r} \in S_{K_{\infty}}^{1}\left(\mathbf{X}^{\mathrm{R}, \alpha}\right)\right)\right)$ under $\alpha>1$ (resp. $\alpha>1$ and $d>2$ ).

Take $\phi \in C_{\infty}\left(\mathbb{R}^{d}\right) \cap\left(\mathcal{F}^{\mathrm{R}, \alpha}\right)_{e}$ with $\left(\int_{\mathbb{R}^{d}}(\phi(x)-\phi(y))^{2} J(x, y) \mathrm{d} y\right) \mathrm{d} x \in \mathbf{K}_{d, \alpha}$ and assume $\alpha>1$. Then $\left(\int_{\mathbb{R}^{d}}(\phi(x)-\phi(y))^{2} J(x, y) \mathrm{d} y\right) \mathrm{d} x \in S_{K_{\infty}^{+}}^{1}\left(\mathbf{X}^{\mathrm{R}, \alpha}\right)$. Take also $F=F_{1}-F_{2}$ with

$$
-1<\inf _{x, y \in \mathbb{R}^{d}} F(x, y) \leq \sup _{x, y \in \mathbb{R}^{d}} F(x, y)<\infty
$$

and suppose $F_{1} \in J_{K_{\infty}^{+}}^{1}\left(\mathbf{X}^{\mathrm{R}, \alpha}\right), F_{2}=0$, or $F_{1} \in J_{K_{\infty}^{+}}^{1}\left(\mathbf{X}^{\mathrm{R}, \alpha}\right), F_{2} \in J_{K_{\infty}}^{1}\left(\mathbf{X}^{\mathrm{R}, \alpha}\right)$ and the transience of $\mathbf{X}^{\mathrm{R}, \alpha}$. For example, for $d=1$ and $F(x, y):=f(x, y)|x-y|^{1+\alpha}$ with a symmetric function $f \in L^{1}\left(\mathbb{R}^{2}\right)$ satisfies this condition provided $f \geq 0$ on $\mathbb{R}^{2}$. We consider another function $F(x, y):=f(x, y)|x-y|^{d+\alpha}$ with a symmetric $f \in L^{1}\left(\mathbb{R}^{2 d}\right)$ satisfying $\int_{\mathbb{R}^{d}} f(x, y) \mathrm{d} y \in L^{p}\left(\mathbb{R}^{d}\right)$ with $p>d / \alpha$ (This condition is satisfied if $f$ has the form $f=f_{1} \otimes f_{2}+f_{2} \otimes f_{1}$ with $\left.f_{1}, f_{2} \in C_{0}\left(\mathbb{R}^{d}\right)\right)$. Then $F$ satisfies the required condition provided $\mathbf{X}^{\mathrm{R}, \alpha}$ is transient.

Consider the following Feynman-Kac semigroup

$$
Q_{t} f(x):=\mathbf{E}_{x}\left[e^{N_{t}^{\phi}+L_{t}^{r}} \operatorname{Exp}\left(A^{F}\right)_{t} f\left(X_{t}\right)\right]
$$


Here $L_{t}^{r}$ is the PCAF in the strict sense associated with the surface measure $\sigma_{r}$. The associated quadratic form $\mathcal{Q}$ is given by

$$
\left\{\begin{array}{r}
\mathcal{Q}(f, g):=\int_{\mathbb{R}^{d} \times \mathbb{R}^{d}}(f(x)-f(y))(g(x)-g(y)) J(x, y) \mathrm{d} x \mathrm{~d} y \\
+\int_{\mathbb{R}^{d} \times \mathbb{R}^{d}}(f g(x)-f g(y))(\phi(x)-\phi(y)) J(x, y) \mathrm{d} x \mathrm{~d} y \\
-\int_{\partial B_{r}(0)} f(x) g(x) \sigma_{r}(\mathrm{~d} x) \\
-\int_{\mathbb{R}^{d} \times \mathbb{R}^{d}} f(x) g(y) F(x, y) J(x, y) \mathrm{d} x \mathrm{~d} y \\
\mathcal{D}(\mathcal{Q}):=\mathcal{F}^{\mathrm{R}, \alpha} .
\end{array}\right.
$$

Let $D$ be a bounded open set with smooth (or more generally regular) boundary. The Lebesgue $m$ on $D$ satisfies $m \in S_{K_{\infty}^{+}}^{1}\left(\mathbf{X}_{D}^{\mathrm{R}, \alpha}\right)$ under $\mathbf{X}_{D}^{\mathrm{R}, \alpha}$ by way of [11, Corollary]. Then we have the following by Theorem 1.1(3):

$$
\lim _{t \rightarrow \infty} \frac{1}{t} \log \sup _{x \in D} \mathbf{E}_{x}\left[e^{N_{t}^{\phi}+L_{t}^{r}} \operatorname{Exp}\left(A^{F}\right)_{t}: t<\tau_{D}\right]=-\inf _{v \in \mathcal{F}_{D}^{\mathrm{R}, \alpha},\|v\|_{2}=1} \mathcal{Q}(v, v) .
$$

Since $\left(|\xi|^{2}+m^{2 / \alpha}\right)^{\alpha / 2}-m=m\left(1+|\xi|^{2} / m^{2 / \alpha}\right)^{\alpha / 2}-m \leq m\left(1+|\xi|^{2} / m^{2 / \alpha}\right)-m=$ $m^{1-\frac{2}{\alpha}}|\xi|^{2}$, we have $H^{1}\left(\mathbb{R}^{d}\right) \subset \mathcal{F}^{\mathrm{R}, \alpha}$ and $\mathcal{E}^{\mathrm{R}, \alpha}(f, f) \leq m^{1-\frac{2}{\alpha}}\|\nabla f\|_{2}^{2}, f \in H^{1}\left(\mathbb{R}^{d}\right)$, hence

$$
\inf _{v \in \mathcal{F}^{\mathrm{R}, \alpha},\|v\|_{2}=1} \mathcal{E}^{\mathrm{R}, \alpha}(v, v)=0 .
$$

Then Corollary 1.2 tells us that the spectral radius $\lambda_{p}\left(\phi, \sigma_{r}, F\right):=-\lim _{t \rightarrow \infty} \frac{1}{t} \log \left\|Q_{t}\right\|_{p, p}$ is independent of $p$ provided $\alpha>1$.

6. Appendix: Fukushima decomposition in the strict sense. In this section, under (AC), without assuming the condition that $\mathbf{X}$ admits no inside killing in the sense that

$$
\mathbf{P}_{x}\left(\zeta<\infty, X_{\zeta-} \in E\right)=0 \text { for all } x \in E,
$$

we will prove that the strict version of Fukushima's decomposition holds for strictly $\mathcal{E}$-quasi continuous function $u$ on $E_{\partial}$ satisfying $u \in \dot{\mathcal{F}}_{\text {loc }}^{\dagger}$ with $\mu_{\langle u\rangle} \in S_{D}^{1}(\mathbf{X})$, which is (nearly) Borel finely continuous on $E$. The condition (6.1) is equivalent to the absence of the killing part in the Beurling-Deny representation of $(\mathcal{E}, \mathcal{F})$ (cf. [15]). In Appendix of [12], we show Fukushima's decomposition (1.1) in the strict sense under (6.1) (Proposition A.1 in [12]), but the conclusion of Proposition A.1 in [12] based on Lemma 3.1 in [6] is not true for the condition $\mu_{\langle u\rangle} \in S_{D}^{1}(\mathbf{X})$ lacking (Lemma 3.1 in [6] is completely wrong). We shall correct Proposition A.1 in [12] in this appendix remaining valid for our main results including [12].

Let $E, E_{\partial}$ and $m$ as in Section 1. Let $\mathbf{X}$ be an $m$-symmetric Markov process Let $(\mathcal{E}, \mathcal{F})$ be the associated symmetric Dirichlet form on $L^{2}(E ; m)$ and assume that $(\mathcal{E}, \mathcal{F})$ is regular.

Denote by $\mathcal{M}_{\text {loc }}^{I(\zeta)}$ (resp. $\stackrel{\mathcal{M}}{\text { ) }}$, the family of locally square integrable martingale additive functionals on $I(\zeta):=\llbracket 0, \zeta \llbracket \cup \llbracket \zeta_{i} \rrbracket$, where $\zeta_{i}$ is the totally inaccessible part of $\zeta$ (resp. of 
square integrable martingale additive functionals of finite energy), and by $\mathcal{N}_{c \text {, loc }}\left(\operatorname{resp} . \mathcal{N}_{c}\right)$ the family of continuous additive functionals locally of zero energy (resp. of continuous additive functionals of zero energy) (see [6, 25, 27]).

THEOREM 6.1 (Generalized Fukushima Decomposition, cf. [25, Theorem 4.2], [26, Theorem 1.2], [27, Theorem 1.4]). For $f \in \dot{\mathcal{F}}_{\text {loc }}^{\dagger}$, the additive functional $A^{f}$ defined by $A_{t}^{f}:=f\left(X_{t}\right)-f\left(X_{0}\right)$ can be decomposed as

$$
A^{f}=M^{f}+N^{f}, \quad M^{f} \in \mathcal{M}_{\mathrm{loc}}^{I(\zeta)}, \quad N^{f} \in \mathcal{N}_{c, \text { loc }}
$$

in the sense that $A_{t}^{f}=M_{t}^{f}+N_{t}^{f}, t \in\left[0, \zeta\left[\mathbf{P}_{x}\right.\right.$-a.s. for q.e. $x \in$ E. Such a decomposition is unique up to the equivalence of additive functionals on $\llbracket 0, \zeta \llbracket$ (or of local additive functionals).

We set $\mathcal{M}_{\mathrm{loc}}^{c, I(\zeta)}:=\left\{M \in \mathcal{M}_{\mathrm{loc}}^{I(\zeta)} \mid M\right.$ is continuous $\}$ and $\mathcal{M}_{\mathrm{loc}}^{d, I(\zeta)}:=\left\{M \in \mathcal{M}_{\mathrm{loc}}^{I(\zeta)} \mid\right.$ $\mathcal{M}_{\mathrm{loc}}^{I(\zeta)}$ is purely discontinuous $\}$. Define

$$
\begin{aligned}
\mathcal{J}:=\{ & \phi: E_{\partial} \times E_{\partial} \rightarrow \mathbb{R} \mid \phi \text { is Borel measurable, } \\
& \text { vanishes on diagonal and } \left.N\left(\phi^{2}\right) \mu_{H} \in S(\mathbf{X})\right\} .
\end{aligned}
$$

For $M \in \mathcal{M}_{\text {loc }}^{d, I(\zeta)}$, there exists $\phi \in \mathcal{J}$ such that $\left.\Delta M_{t}=\phi\left(X_{t-}, X_{t}\right) t \in\right] 0, \zeta\left[\mathbf{P}_{x}\right.$-a.s. for q.e. $x \in E$ (see Theorem 2.1 in [25], Theorem 1.1 in [26]). We set $\mathcal{M}_{\text {loc }}^{j, I(\zeta)}:=\{M \in$ $\mathcal{M}_{\text {loc }}^{d, I(\zeta)} \mid \phi(\cdot, \partial)=0 \kappa$-a.e. on $\left.E\right\}$ and $\mathcal{M}_{\text {loc }}^{\kappa, I(\zeta)}:=\left\{M \in \mathcal{M}_{\text {loc }}^{d, I(\zeta)} \mid \phi=0 J\right.$-a.e. on $\left.E \times E\right\}$.

Let $\mathcal{M}$ be the family of square integrable MAF admitting exceptional set. Note that $\mathcal{M} \subset$ $\mathcal{M} \subset \mathcal{M}_{\text {loc }} \subset \mathcal{M}_{\text {loc }}^{I(\zeta)} \subset \mathcal{M}_{\text {loc }}^{\amalg 0, \zeta \amalg}$. For each $i=c, d, j, \kappa$, we can define $\mathcal{M}^{i}$ analogously as for $\mathcal{M}_{\text {loc }}^{i, I(\zeta)}$. Every $M \in \mathcal{M}$ admits decomposition $M=M^{c}+M^{d}=M^{c}+M^{j}+M^{\kappa}$, where $M^{c} \in \mathcal{M}^{c}, M^{d} \in \mathcal{M}^{d}, M^{j} \in \mathcal{M}^{j}$ and $M^{\kappa} \in \mathcal{M}^{\kappa}$. Set $S_{D}(\mathbf{X}):=\{\mu \in S(\mathbf{X}) \mid$ $\left\|\mathbf{E} .\left[A_{t}^{\mu}\right]\right\|_{q}<\infty$ for some/all $\left.t>0\right\}$, the family of Dynkin class smooth measures. Here $\|f\|_{q}:=\inf _{\operatorname{Cap}(N)=0} \sup _{x \in E \backslash N}|f(x)|$.

Our result is the following:

TheOREM 6.2. Take $f \in \dot{\mathcal{F}}_{\text {loc }}^{\dagger}$ and $\mu_{\langle f\rangle} \in S_{D}(\mathbf{X})$. Assume one of the following:

- $f$ is a strictly $\mathcal{E}$-quasi continuous finite function on $E_{\partial}$, that is, there exists a strict $\mathcal{E}$-nest $\left\{F_{n}\right\}$ of closed sets such that $\left.f\right|_{F_{n} \cup\{\partial\}}$ is continuous on each $F_{n} \cup\{\partial\}$.

- $\mathbf{X}$ is conservative, i.e. $\mathbf{P}_{x}(\zeta=\infty)=1$ q.e. $x \in E$.

Then the following hold:

(1) $A^{f}$ can be decomposed as

$$
A^{f}=M^{f}+N^{f}, M^{f} \in \mathcal{M}, N^{f} \in \mathcal{N}_{c, \text { loc }}^{*}
$$

in the sense that $A_{t}^{f}=M_{t}^{f}+N_{t}^{f}, t \in\left[0, \infty\left[\mathbf{P}_{x}\right.\right.$-a.s. for q.e. $x \in E$. Here $\mathcal{N}_{c, \text { loc }}^{*}:=\left\{N \in \mathcal{N}_{c, \text { loc }} \mid N\right.$ is a CAF admitting exceptional set $\}$. Such a decomposition is unique up to the equivalence of additive functionals. Moreover, $M^{f}$ can 
be decomposed to be

$$
M_{t}^{f}=M_{t}^{f, c}+M_{t}^{f, j}+M_{t}^{f, \kappa} t \in\left[0, \infty\left[\mathbf{P}_{x} \text {-a.s. for q.e. } x \in E,\right.\right.
$$

where $M^{f, c} \in \mathcal{M}^{c}, M^{f, j} \in \mathcal{M}^{j}, M^{f, \kappa} \in \mathcal{M}^{\kappa}$.

(2) Suppose that (AC) holds and $f$ is a (nearly) Borel finely continuous function on $E$. Then next two conditions are equivalent to each other

(a) $\mu_{\langle f\rangle} \in S_{D}^{1}(\mathbf{X})$.

(b) The decomposition

$$
f\left(X_{t}\right)-f\left(X_{0}\right)=M_{t}^{f}+N_{t}^{f}, \quad t \in\left[0, \infty\left[, \quad \mathbf{P}_{x} \text {-a.s. for all } x \in E\right.\right.
$$

holds with

(i) $M^{f} \in \mathcal{M}, M^{f}$ is an $A F$ in the strict sense admitting a PCAF $A$ in the strict sense satisfying $\sup _{x \in E} \mathbf{E}_{x}\left[\int_{0}^{\infty} e^{-t} \mathrm{~d} A_{t}\right]<\infty$ such that

$\mathbf{E}_{x}\left[\left(M_{t}^{f}\right)^{2}\right]=\mathbf{E}_{x}\left[A_{t}\right]<\infty, \quad \mathbf{E}_{x}\left[M_{t}^{f}\right]=0$ for all $x \in E$.

(ii) $N^{f} \in \mathcal{N}_{c, \text { loc }}, N^{f}$ is a CAF in the strict sense.

Moreover, under the equivalence $(2 \mathrm{a}) \Longleftrightarrow(2 \mathrm{~b}), M^{f}$ can be decomposed to be

$$
M_{t}^{f}=M_{t}^{f, c}+M_{t}^{f, j}+M_{t}^{f, \kappa} t \in\left[0, \infty\left[\mathbf{P}_{x} \text {-a.s. for all } x \in E,\right.\right.
$$

where $M^{f, c} \in \mathcal{M}^{c}, M^{f, j} \in \mathcal{M}^{j}, M^{f, \kappa} \in \mathcal{M}^{\kappa}$ are square integrable MAFs in the strict sense. If $f \in \mathcal{F}_{e}$ with $f(\partial)=0, M^{f}$ in (2(b)i) (resp. $N^{f}$ in (2(b)ii)) satisfies $M^{f} \in \stackrel{\circ}{\mathcal{M}}\left(\right.$ resp. $\left.N^{f} \in \mathcal{N}_{c}\right)$.

Proof of Theorem 6.2 (1). For $\phi \in \mathcal{J}$ with $N\left(\phi^{2}\right) \mu_{H} \in S_{D}(\mathbf{X})$, we construct a purely discontinuous MAF $M^{\phi}$ such that $\Delta M_{t}^{\phi}=\phi\left(X_{t-}, X_{t}\right)$ for all $\left.t \in\right] 0, \infty\left[\mathbf{P}_{x}\right.$-a.s. for q.e. $x \in E$.

Set

$$
M_{t}^{(2)}:=\sum_{s \leq t}\left(\mathbf{1}_{\{|\phi|>1\}} \phi\right)\left(X_{s-}, X_{S}\right)-\int_{0}^{t} N\left(\mathbf{1}_{\{|\phi|>1\}} \phi\right)\left(X_{S}\right) \mathrm{d} H_{S} .
$$

Since $N\left(\phi^{2}\right) \mu_{H} \in S_{D}(\mathbf{X})$, we see $\mathbf{E}_{x}\left[\int_{0}^{t} N\left(\phi^{2}\right)\left(X_{S}\right) \mathrm{d} H_{s}\right]<\infty$ for q.e. $x \in E$. Then $M^{(2)}$ is a square integrable MAF. Define an AF $M^{n}$ by

$$
M_{t}^{n}:=\sum_{s \leq t}\left(\mathbf{1}_{\{1 / n<|\phi| \leq 1\}} \phi\right)\left(X_{s-}, X_{S}\right)-\int_{0}^{t} N\left(\mathbf{1}_{\{1 / n<|\phi| \leq 1\}} \phi\right)\left(X_{s}\right) \mathrm{d} H_{s} .
$$

Then it is also a square integrable MAF. For $n>k>1$, we see

$$
\mathbf{E}_{x}\left[\left\langle M^{n}-M^{k}\right\rangle_{t}\right]=\mathbf{E}_{x}\left[\int_{0}^{t} N\left(\mathbf{1}_{\{1 / n<|\phi| \leq 1 / k\}} \phi^{2}\right)\left(X_{s}\right) \mathrm{d} H_{s}\right] .
$$

Then Doob's maximal inequality yields that there exists a subsequence $\left\{n_{k}\right\}$ such that $M_{t}^{n_{k}}$ converges uniformly on each compact subinterval of $\left[0, \infty\left[\mathbf{P}_{x}\right.\right.$-a.s. for q.e. $x \in E$. Therefore the limit

$$
M_{t}^{(1)}:=\lim _{k \rightarrow \infty} M_{t}^{n_{k}}
$$


exists and defines a square integrable MAF. Thus, $M_{t}^{\phi}:=M_{1}^{(1)}+M_{t}^{(2)}$ is the desired square integrable MAF. We then set $M^{f, d}:=M^{\phi}$ for $\phi(x, y):=f(y)-f(x), M^{f, j}:=M^{\phi}$ for $\phi(x, y):=\mathbf{1}_{E \times E}(x, y)(f(y)-f(x))$ and $M^{f, \kappa}:=M^{\phi}$ for $\phi(x, y):=f(\partial)-f(x)$. Note here that there is no need to define $M^{f, \kappa}$ if $\mathbf{X}$ is conservative.

Next we construct the continuous square integrable MAF $M^{f, c}$ whose quadratic variational process $\left\langle M^{f, c}\right\rangle$ is a PCAF associated with $\mu_{\langle f\rangle}^{c} \in S_{D}$. Take $\left\{f_{n}\right\} \subset \mathcal{F}$ and a nest $\left\{G_{n}\right\}$ of finely open (nearly) Borel sets such that $f=f_{n} m$-a.e. on $G_{n}$. The strongly local properties of $\mu_{\langle f\rangle}^{c}$ and $\mu_{\left\langle f_{n}\right\rangle}^{c}$ imply $\mathbf{1}_{G_{k}} \mu_{\langle f\rangle}^{c}=\mathbf{1}_{G_{k}} \mu_{\left\langle f_{k}\right\rangle}^{c}=\mathbf{1}_{G_{k}} \mu_{\left\langle f_{n}\right\rangle}^{c}=\mathbf{1}_{G_{k}} \mu_{\left\langle f_{n}, f_{k}\right\rangle}^{c}$ for each $n, k \in \mathbb{N}$ with $k<n$. Let $(g * M)_{t} \in \stackrel{\circ}{\mathcal{M}}$ denotes the stochastic integral by an MAF $M \in \stackrel{\circ}{\mathcal{M}}$ with $g \in L^{2}\left(E ; \mu_{\langle M\rangle}\right) \cap \mathcal{B}(E)$ constructed in Theorem 5.6.1 in [16]. Note that $\mathbf{1}_{G_{n}} * M^{f_{n}, c}$ forms a continuous MAF of finite energy for each $n \in \mathbb{N}$. Let $A$ be a PCAF associated with $\mu_{\langle f\rangle}^{c}$. Then $\left(\mathbf{1}_{G_{k}} * A\right)_{t}=\left(\mathbf{1}_{G_{k}} *\left\langle M^{f_{k}, c}\right\rangle\right)_{t}=\left(\mathbf{1}_{G_{k}} *\left\langle M^{f_{n}, c}\right\rangle\right)_{t}=\left(\mathbf{1}_{G_{k}} *\left\langle M^{f_{n}, c}, M^{f_{k}, c}\right\rangle\right)_{t}$ for $k<n$. Here $(g * B)_{t}$ denotes the PCAF defined by $\int_{0}^{t} g\left(X_{S}\right) \mathrm{d} B_{s}$ for $g \in \mathcal{B}_{+}(E)$ and a PCAF $B$. We then see that for $k<n$

$$
\begin{aligned}
\mathbf{E}_{x}\left[\left(\mathbf{1}_{G_{n}} * M_{t}^{f_{n}, c}-\mathbf{1}_{G_{k}} * M_{t}^{f_{k}, c}\right)^{2}\right] & =\mathbf{E}_{x}\left[\left\langle\mathbf{1}_{G_{n}} * M^{f_{n}, c}-\mathbf{1}_{G_{k}} * M^{f_{k}, c}\right\rangle_{t}\right] \\
& =\mathbf{E}_{x}\left[\left\langle\mathbf{1}_{G_{n}} * M^{f_{n}, c}\right\rangle_{t}-\left\langle\mathbf{1}_{G_{k}} * M^{f_{k}, c}\right\rangle_{t}\right] \\
& =\mathbf{E}_{x}\left[\left(\mathbf{1}_{G_{n}} *\left\langle M^{f_{n}, c}\right\rangle\right)_{t}-\left(\mathbf{1}_{G_{k}} *\left\langle M^{f_{k}, c}\right\rangle\right)_{t}\right] \\
& \leq \mathbf{E}_{x}\left[\int_{0}^{t} \mathbf{1}_{G_{n}}\left(X_{s}\right) \mathrm{d} A_{s}-\int_{0}^{t} \mathbf{1}_{G_{k}}\left(X_{s}\right) \mathrm{d} A_{s}\right] \\
& =\mathbf{E}_{x}\left[\int_{0}^{t} \mathbf{1}_{G_{n} \backslash G_{k}}\left(X_{s}\right) \mathrm{d} A_{s}\right] \leq \mathbf{E}_{x}\left[A_{t}\right]<\infty
\end{aligned}
$$

for q.e. $x \in E$. Then Doob's maximal inequality yields that there exists a subsequence $\left\{n_{k}\right\}$ such that $\mathbf{1}_{G_{n_{k}}} * M_{t}^{f_{n_{k}}, c}$ converges uniformly on each compact subinterval of [0, $\infty\left[\mathbf{P}_{x}\right.$-a.s. for q.e. $x \in E$. We then see that the limit $M_{t}^{f, c}:=\lim _{k \rightarrow \infty} \mathbf{1}_{G_{n_{k}}} * M_{t}^{f_{n_{k}}, c}$ forms a square integrable continuous MAF. We see $M_{t}^{f, c}=M_{t}^{f_{n}, c} t<\tau_{G_{n}} \mathbf{P}_{x}$-a.s. for q.e. $x \in E$.

Now we set $N_{t}^{f}:=f\left(X_{t}\right)-f\left(X_{0}\right)-M_{t}^{f, c}-M_{t}^{f, d}$. We see that $N^{f}$ is an AF admitting exceptional set. Assume first the strict $\mathcal{E}$-quasi-continuity of $f$ on $E_{\partial}$. Then, there exists a strict $\mathcal{E}$-nest $\left\{F_{n}\right\}$ of closed sets such that $F_{n} \cup\{\partial\}$ is compact in $E_{\partial}$ and $\left.f\right|_{F_{n}} \cup\{\partial\}$ is continuous on $F_{n} \cup\{\partial\}$ for each $n \in \mathbb{N}$. Noting $\mathbf{P}_{x}\left(\lim _{n \rightarrow \infty} \sigma_{E \backslash F_{n}}=\infty\right)=1$ for q.e. $x \in E$, we have that $\lim _{s \downarrow t} f\left(X_{S}\right)=f\left(X_{t}\right)$ for all $t \in\left[0, \infty\left[\right.\right.$ and $\lim _{s \uparrow t} f\left(X_{s}\right)=f\left(X_{t-}\right)$ for all $\left.t \in\right] 0, \infty[$ $\mathbf{P}_{x}$-a.s. for q.e. $x \in E$. Then we obtain that $N^{f}$ is a CAF admitting exceptional set under the strict $\mathcal{E}$-quasi-continuity of $f$ on $E_{\partial}$. It is clear that the same conclusion also holds under the conservativeness of $\mathbf{X}$, because $f$ is always $\mathcal{E}$-quasi-continuous on $E$. Finally, we prove the uniqueness of the decomposition: It suffices to prove $\mathcal{M} \cap \mathcal{N}_{c \text {,loc }}^{*}=\{0\}$. Take $M \in \mathcal{M}$ and $N \in \mathcal{N}_{c, \text { loc }}^{*}$. Suppose that $M_{t}+N_{t}=0$ for all $t \in\left[0, \infty\left[\mathbf{P}_{x}\right.\right.$-a.s. for q.e. $x \in E$. Owing to the uniqueness of the decomposition in Theorem 6.1, we have $M_{t}=N_{t}=0$ for $t \in[0, \zeta$ [ $\mathbf{P}_{x}$-a.s. for q.e. $x \in E$. Since $N \in \mathcal{N}_{c, \text { loc }}^{*}$ is a CAF admitting exceptional set, $N_{t}=N_{\zeta}=0$ for all $t \in\left[\zeta, \infty\left[\mathbf{P}_{x}\right.\right.$-a.s. for q.e. $x \in E$. This implies the desired uniqueness. 
Proof of $(2 \mathrm{a}) \Longrightarrow(2 \mathrm{~b})$ : The proof is a repetition of the proof of (1). For $\phi \in \mathcal{J}$ with $N\left(\phi^{2}\right) \mu_{H} \in S_{D}^{1}(\mathbf{X})$, we can construct a square integrable purely discontinuous MAF $M^{\phi}$ in the strict sense such that $\Delta M_{t}^{\phi}=\phi\left(X_{t-}, X_{t}\right)$ for all $t \in\left[0, \infty\left[\mathbf{P}_{x}\right.\right.$-a.s. for all $x \in E$. Consequently, $M^{f, d}, M^{f, j}, M^{f, \kappa}$ can be constructed as square integrable MAFs in the strict sense under $f \in \dot{\mathcal{F}}_{\text {loc }}^{\dagger}$ and $\mu_{\langle f\rangle} \in S_{D}^{1}(\mathbf{X})$ under the conditions (there is no need to define $M^{f, \kappa}$ if $\mathbf{X}$ is conservative). Similarly as in the proof of (1), we can construct continuous square integrable MAF $M_{t}^{f, c}$ in the strict sense. Assume first the strict $\mathcal{E}$-quasi-continuity of $f$ on $E_{\partial}$. Take a strict $\mathcal{E}$-nest $\left\{F_{n}\right\}$ of closed sets such that $F_{n} \cup\{\partial\}$ is compact in $E_{\partial}$ and $\left.f\right|_{F_{n} \cup\{\partial\}}$ is continuous on $F_{n} \cup\{\partial\}$ for each $n \in \mathbb{N}$. Noting $\mathbf{P}_{x}\left(\lim _{n \rightarrow \infty}\left(t+\sigma_{E \backslash F_{n}} \circ \theta_{t}\right)=\infty\right)=1$ for all $x \in E$, we have that $\lim _{s \downarrow t} f\left(X_{s}\right)=f\left(X_{t}\right)$ for all $t \in\left[0, \infty\left[\right.\right.$ and $\lim _{s \uparrow t} f\left(X_{S}\right)=f\left(X_{t-}\right)$ for all $t \in] 0, \infty\left[\mathbf{P}_{x}\right.$-a.s. for all $x \in E$. The same conclusion also holds under the conservativeness of $\mathbf{X}$. Moreover, the quantity $f\left(X_{0}\right)$ is uniquely determined in the sense that $\mathbf{P}_{x}$-a.s. for all $x \in E$ under the fine continuity of $f$ on $E$ with (AC), because $f_{1}=f_{2}$ on $E$ provided $f_{1}=f_{2}$ q.e. (or $m$-a.e.) on $E$ for any finely continuous (nearly) Borel functions $f_{1}, f_{2}$ on $E$. Note that $f\left(X_{0}\right)$ is only uniquely determined in the sense that $\mathbf{P}_{x}$-a.s. for q.e. $x \in E$ for any $\mathcal{E}$-quasi continuous function $f$ on $E$. Then $N_{t}^{f}:=f\left(X_{t}\right)-f\left(X_{0}\right)-M^{f, c}-M_{t}^{f, d}$ forms a $\mathrm{CAF}$ in the strict sense. Other properties in (2b) can be easily confirmed.

Proof of $(2 \mathrm{~b}) \Longrightarrow(2 \mathrm{a})$ : Let $M^{f}, N^{f}$ and $A$ be functionals satisfying condition (2b) of Theorem 6.2. Then $M^{f}$ and $N^{f}$ are strict versions of AFs appeared in the decomposition in (1) and we can see that $A$ is a strict version of $\left\langle M^{f}\right\rangle$. Hence $\mu_{\langle f\rangle}$ is the Revuz measure of the PCAF $A$ in the strict sense and we see $\mu_{\langle f\rangle} \in S_{1}(\mathbf{X})$ with $\sup _{x \in E} R_{1} \mu_{\langle f\rangle}(x)<\infty$, which shows $\mu_{\langle f\rangle} \in S_{D}^{1}(\mathbf{X})$.

Acknowledgment. The authors would like to thank Professor Masayoshi Takeda for his valuable comment on a draft of this paper.

\section{REFERENCES}

[ 1 ] R. Azencott, Behavior of diffusion semi-groups at infinity, Bull. Soc. Math. France 102 (1974), 193-240.

[2] R. M. Blumenthal and R. K. Getoor, Some theorems on stable processes, Trans. Amer. Math. Soc. 95 (1960), 263-273.

[ 3 ] Z.-Q. CHEN, Uniform integrability of exponential martingales and spectral bounds of non-local FeynmanKac semigroups, Stochastic Analysis and Applications to Finance, Essays in Honor of Jia-an Yan. Eds by T. Zhang and $\mathrm{X}$. Zhou, 2012.

[ 4 ] Z.-Q. CHEN, $L^{p}$-independence of spectral bounds of generalized non-local Feynman-Kac semigroups, J. Funct. Anal. 262 (2012), no. 9, 4120-4139.

[ 5 ] Z.-Q. Chen, P. J. Fitzsimmons, M. Takeda, J. Ying And T.-S. Zhang, Absolute continuity of symmetric Markov processes, Ann. Probab. 32 (2004), no. 3, 2067-2098.

[6] Z.-Q. Chen, P. J. Fitzsimmons, K. KuwaE And T.-S. Zhang, Stochastic calculus for symmetric Markov processes, Ann. Probab. 36 (2008), no. 3, 931-970.

[ 7 ] Z.-Q. Chen, P. J. Fitzsimmons, K. Kuwae And T.-S. Zhang, On general perturbations of symmetric Markov processes, J. Math. Pures et Appliquées 92 (2009), no. 4, 363-374.

[ 8 ] Z.-Q. CHEN AND K. KuwAE, On doubly Feller property, Osaka J. Math. 46, (2009), no. 4, 909-930.

[9] Z.-Q. CHEN AND R. SONG, Drift transforms and Green function estimates for discontinuous processes, J. 
Funct. Anal. 201 (2003), no. 1, 262-281.

[10] Z.-Q. Chen AND T.-S. ZHANG, Girsanov and Feynman-Kac type transformations for symmetric Markov processes, Ann. Inst. H. Poincaré Probab. Statist. 38 (2002), no. 4, 475-505.

[11] K. L. Chung, Doubly-Feller process with multiplicative functional, Seminar on stochastic processes, 1985 (Gainesville, Fla., 1985), 63-78, Progr. Probab. Statist. 12, Birkhäuser Boston, Boston, MA, 1986.

[12] G. DE LEva, D. KIm AND K. KUWAE, $L^{p}$-independence of spectral bounds of Feynman-Kac semigroups by continuous additive functionals, J. Funct. Anal. 259 (2010), no. 3, 690-730.

[13] W. Feller, The birth and death processes as diffusion processes, J. Math. Pures Appl. (9) 38 (1959), 301-345.

[14] P. J. Fitzsimmons, Absolute continuity of symmetric diffusions, Ann. Probab. 25 (1997), no. 1, 230-258.

[15] M. Fukushima, On a decomposition of additive functionals in the strict sense for a symmetric Markov process, Dirichlet forms and stochastic processes (Beijing, 1993), 155-169, de Gruyter, Berlin, 1995.

[16] M. Fukushima, Y. Oshima And M. Takeda, Dirichlet Forms and Symmetric Markov Processes. Second revised and extended edition. de Gruyter Studies in Mathematics, 19. Walter de Gruyter \& Co., Berlin, 1994.

[17] M. Fukushima And M. TAKedA, A transformation of a symmetric Markov process and the DonskerVaradhan theory, Osaka J. Math. 21 (1984), no. 2, 311-326.

[18] I. W. Herbst AND A. D. SloAn, Perturbation of translation invariant positivity preserving semigroups on $L^{2}\left(\mathbb{R}^{n}\right)$, Trans. Amer. Math. Soc. 236 (1978), 325-360.

[19] D. KIM, Asymptotic properties for continuous and jump type's Feynman-Kac functionals, Osaka J. Math. 37 (2000), no. 1, 147-173.

[20] D. KIM AND K. KUWAE, Analytic characterizations of gaugeability for generalized Feynman-Kac functionals, (2016), to appear in Transactions of AMS.

[21] D. Kim, M. TAKedA AND J. YING, Some variational formulas on additive functionals of symmetric Markov chains, Proc. Amer. Math. Soc. 130 (2002), no. 7, 2115-2123.

[22] K. Kuwae And M. TAKAhashi, Kato class functions of Markov processes under ultracontractivity, Potential theory in Matsue, 193-202, Adv. Stud. Pure Math. 44, Math. Soc. Japan, Tokyo, 2006.

[23] K. Kuwae and M. TaKahashi, Kato class measures of symmetric Markov processes under heat kernel estimates, J. Funct. Anal. 250 (2007), no. 1, 86-113.

[24] K. Kuwae And S. NaKao, Time changes in Dirichlet space theory, Osaka J. Math. 28 (1991), no. 4, 847865.

[25] K. KUwAE, Stochastic calculus over symmetric Markov processes without time reversal, Ann. Probab. 38 (2010), no. 4 1532-1569.

[26] K. KUWAE, Errata Stochastic calculus over symmetric Markov processes without time reversal, Ann. Probab. 40 (2012), no. 6, 2705-2706.

[27] Z.-M. MA, W. Sun AND L.-F. WANG, Fukushima type decomposition for semi-Dirichlet forms, Tohoku Math. J. 68 (2016), no. 1, 1-27.

[28] Y. Ogura AND M. Tomisaki, One dimensional diffusion processes, Abstracts of Summer School of Probability Theory, Kyushu University. (2004) (in Japanese). http://www.math.kyotou.ac.jp/probability/sympo/PSS04.html

[29] S. C. PORT, The first hitting distribution of a sphere for symmetric stable processes, Trans. Amer. Math. Soc. 135 (1969), 115-125.

[30] M. Ł. RYZNAR, Estimates of Green function for relativistic $\alpha$-stable process, Potential Anal. 17 (2002), no. 1, $1-23$.

[31] P. Stollmann And J. Voigt, Perturbation of Dirichlet forms by measures, Potential Anal. 5 (1996), no. 2, 109-138.

[32] M. TAKEDA, On a large deviation for symmetric Markov processes with finite life time, Stochastics Stochastic Reports 59 (1996), no. 1-2, 143-167.

[33] M. TAKEDA, Asymptotic properties of generalized Feynman-Kac functionals, Potential Anal. 9 (1998), no. 3, 261-291. 
[34] M. TAKEDA, $L^{p}$-independence of the spectral radius of symmetric Markov semigroups, Stochastic processes, physics and geometry: new interplays, II (Leipzig, 1999), 613-623, CMS Conf. Proc. 29, Amer. Math. Soc., Providence, RI, 2000.

[35] M. TAKEDA, Conditional gaugeability and subcriticality of generalized Schrödinger operators, J. Funct. Anal. 191 (2002), no. 2, 343-376.

[36] M. TAKEDA, $L^{p}$-independence of spectral bounds of Schrödinger type semigroups, J. Funct. Anal. 252 (2007), no. 2, 550-565.

[37] M. TAKedA, A large deviation principle for symmetric Markov processes with Feynman-Kac functional, J. Theoret. Probab. 24 (2011), no. 4, 1097-1129.

[38] M. TAKEDA, $L^{p}$-independence of growth bounds of Feynman-Kac semigroups, Surveys in Stochastic Processes, eds. J. Blath, P. Imkeller, S. Roelly, Proceedings of the 33rd SPA Conference in Berlin, 2009. 201226, EMS Ser. Congr. Rep., Eur. Math. Soc., Zürich, 2011.

[39] M. TAKEDA AND Y. TAWARA, $L^{p}$-independence of spectral bounds of non-local Feynman-Kac semigroups, Forum Math. 21 (2009), no. 6, 1067-1080.

[40] M. TAKeda AND Y. TAWARA, A large deviation principle for symmetric Markov processes normalized by Feynman-Kac functionals, Osaka J. Math. 50 (2013), no. 2, 287-307.

[41] M. TAKedA AND T.-S. ZhANG, Asymptotic properties of additive functionals of Brownian motion, Ann. Probab. 25 (1997), no. 2, 940-952.

[42] Y. TAWARA, $L^{p}$-independence of spectral bounds of Schrödinger type operators with non-local potentials, J. Math. Soc. Japan 62 (2010), no. 3, 767-788.

[43] Y. TAWARA, $L^{p}$-independence of growth bounds of generalized Feynman-Kac semigroups, Doctor's Degree Thesis, Mathematical Institute, Tohoku University, 2009.

[44] J. YING, Dirichlet forms perturbated by additive functionals of extended Kato class, Osaka J. Math. 34 (1997), no. 4, 933-952.

[45] T.-S. ZHANG, Generalized Feynman-Kac semigroups, associated quadratic forms and asymptotic properties, Potential Anal. 14 (2001), no. 4, 387-408.

Department of Mathematics AND ENGINEERING

GraduATE SCHOOL OF SCIENCE AND TECHNOLOGY

KUMAMOTO UNIVERSITY

KUMAMOTO 860-8555

JAPAN

E-mail address: daehong@gpo.kumamoto-u.ac.jp

Division OF GENERAL EDUCATION

Nagaoka National College of Technology

888 NISHIKATAKAI, NAGAOKA

NIIGATA 940-8532

JAPAN

E-mail address: tawara@nagaoka-ct.ac.jp
Department of Mathematics

FACULTY OF SCIENCE

FUKUOKA UNIVERSITY

FUKUOKA 814-0180

JAPAN

E-mail address: kuwae@fukuoka-u.ac.jp 